\title{
A Hybrid Molecular Dynamics Kinetic Monte Carlo Simulation Methodology
}

by

Peter A Zoontjens

\author{
A thesis \\ submitted to the Victoria University of Wellington \\ in fulfilment of the \\ requirements for the degree of \\ Doctor of Philosophy \\ in Physics.
}

Victoria University of Wellington

2014 


\begin{abstract}
This thesis describes a novel hybrid computational methodology in which the Molecular Dynamics and Kinetic Monte Carlo methods are concurrently combined. This hybrid methodology has been developed to simulate phenomena which are unfeasible to treat with either Molecular Dynamics or Kinetic Monte Carlo alone, due to the wide range of time scales involved and the need for highly detailed atom dynamics. Is is shown that the hybrid methodology can reproduce the results of a larger (more atoms) all Molecular Dynamics simulation at a significant reduction in computational cost (run time) - due to the replacement of Molecular Dynamics atoms with Kinetic Monte Carlo atoms.

The hybrid methodology has been successfully used to study the dynamics of epitaxial stacking fault grain boundaries. This work identified that grain boundary motion was hindered by atoms lodging in off-lattice sites, and also by overlayer islands built up by adatom deposition. It was verified that the "kink flip" move is a key element in the motion of grain boundaries.

Methods for enhancing the hybrid methodology were researched. It was shown that by an optimal choice of damping parameter $\gamma$, wave reflections back into the Molecular Dynamics domain could be minimised. This is expected to enable the hybrid methodology to operate successfully with smaller Molecular Dynamics domains, making larger and/or longer simulation runs feasible. This research included the derivation of the dispersion relation for the discrete case with damping and net reflectivity formulas. These are believed to be new results.

The hybrid model can be applied to a wide variety of MD and KMC methods. Other MD potentials such as Embedded Atom or Modified Embedded Atom could be employed. The KMC component can be developed to use a more refined lattice or an "on the fly" KMC method could be employed. Both the MD and KMC
\end{abstract}


components can be extended to handle more than one species of atom. Parallelised versions of the MD and KMC components could also be developed.

Any situation where the problem can be decomposed into distinct domains of fine scale and coarse scale modelling respectively, is potentially suitable for treatment with a hybrid model of this design. 


\section{Acknowledgement}

I would very much like to thank my supervisors Shaun Hendy and Richard Tilly for all their help, direction and advice, in the research and work that lead to the successful conclusion of this thesis. Also I am grateful for the comradeship of the students, post docs and staff in the IRL applied maths division. Thanks also to Ian and Salvy for allowing me some time to finish off the thesis. Last but by no means least, I am deeply indebted to my family for all their understanding, support and encouragement throughout this long process of completing my $\mathrm{PhD}$. 


\section{Contents}

1 Introduction 1

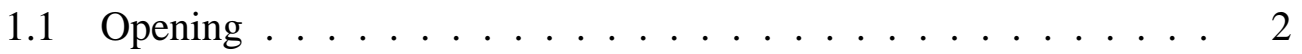

1.1.1 Epitaxial Growth . . . . . . . . . . . . . 2

1.1.2 Significance of Epitaxial Structures . . . . . . . . 2

1.1.3 Modelling of Epitaxial Growth . . . . . . . . . . . . 3

1.1.4 Hybrid Models . . . . . . . . . . . . . . . 4

1.2 Chapter Overviews . . . . . . . . . . . . . . 5

1.2.1 Chapter 2: Epitaxial Growth . . . . . . . . . . 5

1.2.2 Chapter 3: Methodologies ............. 5

1.2.3 Chapter 4: Molecular Dynamics . . . . . . . . . . . 5

1.2.4 Chapter 5: Hybrid Modelling of Grain Boundaries . . . . 6

1.2.5 Chapter 6: Model Enhancement . . . . . . . . . 6

1.3 Summary . . . . . . . . . . . . . . . . . . 7

2 Epitaxial Growth $\quad 8$

2.1 Introduction . . . . . . . . . . . . . . . . . 9

2.2 Epitaxial Growth . . . . . . . . . . . . . . . . . . 9

2.3 Technological Importance $\ldots \ldots . . \ldots 9$

2.3.1 Applications of epitaxy . . . . . . . . . . . . 10

2.4 Features of Epitaxial Growth . . . . . . . . . . . . . . . . . 11

2.4.1 Homo-and Hetero-Epitaxy . . . . . . . . . . . . . . 11

2.4.2 Deposition Methods . . . . . . . . . . . . . 11

2.4 .3 Surface analysis . . . . . . . . . . . . . 12 
2.4.4 Epitaxial Growth Modes . . . . . . . . . . . . . . 13

2.4.5 Layer Growth . . . . . . . . . . . . . . . . . 14

2.5 Modelling Epitaxial Growth . . . . . . . . . . . . 16

2.5.1 Atomistic Modelling Methodologies . . . . . . . . . . 16

2.5.2 Continuum Modelling Methodologies . . . . . . . . . . 17

2.6 Hybrid Methods . . . . . . . . . . . . . . . . . . . . . . . . . . . 19

3 Modelling Methodologies $\quad 21$

3.1 Introduction . . . . . . . . . . . . . . . . . . 22

3.2 Molecular Dynamics . . . . . . . . . . . . . . . . 22

3.2.1 Applications of MD . . . . . . . . . . . 22

3.2.2 MD Equations of Motion . . . . . . . . . . . . . 23

3.2 .3 Time Integration . . . . . . . . . . . . . . . . . . . 24

3.2.4 Periodic Boundary Conditions . . . . . . . . . . 25

3.2.5 Temperature Control . . . . . . . . . . . . . . 26

3.2.6 The Lennard-Jones Potential . . . . . . . . . . . . . 27

3.2.7 The Embedded Atom Method . . . . . . . . . . . . . 30

3.2.8 Integration Time Step . . . . . . . . . . . . . . 31

3.2.9 Computational Cost . . . . . . . . . . . . 33

3.2 .10 Visualisation . . . . . . . . . . . . . 34

3.2.11 Combining Methodologies . . . . . . . . . . . 35

3.3 Transition State Theory $(\mathrm{TST}) \ldots \ldots . \ldots . \ldots 36$

3.3 .1 TST Assumptions . . . . . . . . . . . 36

3.3 .2 Harmonic TST . . . . . . . . . . . . . 38

3.4 The Nudged Elastic Band method . . . . . . . . . . . . . . . 39

3.4.1 Finding the Minimum Energy Path . . . . . . . . . . . . 39

3.4 .2 Enhancements . . . . . . . . . . . . 40

3.5 Simulation Time Scale . . . . . . . . . . . . . . . . . 43

3.5.1 Accelerated Molecular Dynamics . . . . . . . . . . . 43

3.5.2 Autonomous Basin Climbing Method . . . . . . . . . . 46

3.6 Kinetic Monte Carlo . . . . . . . . . . . . . . . . . . . . . 47 
3.6.1 Uses and Features of the KMC Methodology . . . . . . . 47

3.6 .2 Basic Idea of KMC . . . . . . . . . . . . . . 48

3.6.3 KMC Scheme for Crystal Growth . . . . . . . . . . . . . 49

3.6 .4 KMC Algorithm . . . . . . . . . . . . . . . 50

3.6.5 Events and Energy Barriers . . . . . . . . . . . 51

3.7 Summary . . . . . . . . . . . . . . . 53

4 Molecular Dynamics Modelling $\quad 55$

4.1 Molecular Dynamics Modelling . . . . . . . . . . . . 56

4.2 Atomic Layer Stacking on the (111) Facet . . . . . . . . . . . . 56

4.3 Stacking Fault Grain Boundaries . . . . . . . . . . . . . . . 62

4.3 .1 Slab of Atoms . . . . . . . . . . . . . . 62

4.3.2 FCC and HCP site energies . . . . . . . . . . . 62

4.3.3 Initial Configuration . . . . . . . . . . . . 63

4.4 Grain Boundary Shape . . . . . . . . . . . . . . . . 63

4.5 Atomic Details of Grain Boundary Motion . . . . . . . . . . . 70

4.6 Grain Boundary Migration . . . . . . . . . . . . . . . . 80

4.6.1 Changes in Grain Boundary Position . . . . . . . . . . . 80

4.6.2 Random Walk . . . . . . . . . . . . . . . . 81

4.6.3 Temperature Dependence . . . . . . . . . . . . . 83

4.6.4 Grain Boundary Collision . . . . . . . . . . . . 85

4.6.5 Drift ..................... 85

4.7 Occurrence of Atomic Rearrangements . . . . . . . . . . . . . 88

4.8 Summary . . . . . . . . . . . . . . . . . . . . . 91

5 Hybrid MD KMC Model 92

5.1 Hybrid Methodology . . . . . . . . . . . . . . . . 93

5.2 Features of the Hybrid Method . . . . . . . . . . . . . . . 93

5.2.1 Methodology Domains . . . . . . . . . . . . . 93

5.2 .2 Domain Interface . . . . . . . . . . . . . . . . 94

5.3 Initial Configuration . . . . . . . . . . . . . . . . . 95

5.3.1 Arrangement of Atoms . . . . . . . . . . . . 95 
5.3.2 Domain Geometry . . . . . . . . . . . . 95

5.3 .3 MD Domain Length . . . . . . . . . . . . . . . . 98

$5.3 .4 \quad$ KMC Lattice . . . . . . . . . . . . . . . . . . . . . 101

5.4 Hybrid Algorithm - Domain Adaptivity . . . . . . . . . . . . 102

5.4.1 Algorithm . . . . . . . . . . . . . . . . 102

5.4 .2 Initialisation . . . . . . . . . . . . . 103

5.4 .3 Initial Domain Identification . . . . . . . . . . . 104

5.4 .4 Dynamic Steps . . . . . . . . . . . . . . . . . 104

5.4.5 Total Simulation Run-Time . . . . . . . . . . . . . . . 107

5.5 Off-Lattice Events . . . . . . . . . . . . . . . . . . . . . . 108

5.6 Effect of Proximity to a KMC Domain . . . . . . . . . . . . 108

5.7 Grain Boundaries and Adatoms . . . . . . . . . . . . . . 110

5.7.1 Pinning by Adatoms . . . . . . . . . . . . . . 113

5.7.2 Trapping by Adislands . . . . . . . . . . . . . . 113

5.8 Effect of Varying Langevin Parameter $\gamma$. . . . . . . . . . . . . 114

5.9 Benefits of the Hybrid Scheme . . . . . . . . . . . . . . 121

5.9.1 Computational Cost Reduction . . . . . . . . . . . . . 121

5.9.2 Active Simulation in KMC Domains . . . . . . . . . . . 123

5.10 Summary . . . . . . . . . . . . . . . . . 123

6 Enhancement 125

6.1 Introduction . . . . . . . . . . . . . . . . . 126

6.2 Wave Reflections . . . . . . . . . . . . . . . 126

6.3 Equation of Motion and Dispersion Relation . . . . . . . . . . 127

6.4 Reflection of Waves . . . . . . . . . . . . . . . . . . 128

6.4.1 Reflection Coefficient $R_{c} \ldots \ldots \ldots$. . . . . 129

6.5 Dispersion Relation with Damping . . . . . . . . . . . . . 131

$6.5 .1 \quad k$ as a Function of $\omega \ldots \ldots . \ldots 137$

6.5.2 Damping Effect . . . . . . . . . . . . . . . . 141

6.5.3 Damped Reflection Coefficient . . . . . . . . . . . . . 142

6.6 Reflection with a Damping Region . . . . . . . . . . . . . . 146 
6.7 Hybrid Model with a Damping Region . . . . . . . . . . . . 155

6.7.1 Selection of $\gamma$ in the Damping Region . . . . . . . . . 158

6.8 Summary . . . . . . . . . . . . . . . . . . . 160

7 Discussion and Conclusion 161

7.1 Discussion . . . . . . . . . . . . . . . . . 162

7.1.1 Chapter Summaries . . . . . . . . . . . . . . . 163

7.2 Conclusion . . . . . . . . . . . . . . 165 


\section{Chapter 1}

\section{Introduction}




\subsection{Opening}

\subsubsection{Epitaxial Growth}

The term epitaxy, which was coined by Royer [1], comes from the Greek roots epi, meaning "above", and taxis, meaning "in ordered manner", which can be translated as "to arrange upon". Epitaxial growth occurs when crystalline material deposited on a crystalline substrate adopts the crystallographic structure of the substrate. The deposition is typically by way of a flux of individual atoms. Epitaxial growth may result in the deposit forming a continuous film - referred to as an epitaxial film or epitaxial layer, or distinct islands [2], or a combination [3]. Epitaxial growth can be used to produce crystalline thin films of well defined thickness. As an extreme example, the Atomic Layer Epitaxy technique can produce compound semiconductor films a single atomic layer thick [4]. Epitaxial growth of thin films has now become a very important element in the fabrication of electronic and optical devices, because modern semiconductor devices are typically composed of assemblies of numerous thin layers of distinct compositions "heterostructures".

\subsubsection{Significance of Epitaxial Structures}

Pashley [5] has given an historical review of early work on epitaxial growth. Epitaxial growth first became of interest to the semiconductor industry in the 1960s when Theurer [6] demonstrated the growth of thin silicon epitaxial layers on a silicon substrate. It was later suggested by Esaki and Tsu [7] that epitaxial structures could lead to new electronic and optical phenomena. This relied on forming two dimensional semiconductor structures - "quantum wells", which would confine carriers within regions in the order of their de Broglie wavelength, whereupon quantum effects were expected to become evident. Confinement in a quantum well was first observed in 1974 by Dingle [8].

One major application of semiconductor epitaxial thin film structures is in optoelectronic devices including light emitting diodes and lasers. These devices 
are used in fiber optical communication systems and optical data storage. Improvements in the quality of epitaxial GaN layers enabled the development of a very bright blue GaN light emitting diode $[9,10]$. The long-lived InGaN blue light laser [11] utilises multiple quantum wells. Use of blue light significantly increases the storage density of optical media. Considerable work has gone into producing heterostructures that can confine carriers to one dimension - quantum wires, and to zero dimensions - quantum dots, as a way to develop improved devices. For example, quantum dot lasers are expected to have significantly improved threshold current and modulation dynamics compared to quantum well lasers [12]. The expectation of high carrier mobility in quantum wires suggests they may have a role in high speed devices [13].

Epitaxial structures also play a significant role in basic science. Epitaxy of metal-metal systems was undertaken with the expectation that high-quality metallic thin film structures would exhibit novel magnetic phenomena. This expectation was fufilled leading to the discovery of enhanced magnetoresistance [14] and giant magnetoresistance [15]. Epitaxial structures are used to test fundamental ideas in condensed matter physics such as the Wigner electron crystal [16, 17]. This lead to the discovery of the quantum hall effect [18] and subsequently the fractional quantum hall effect [19].

\subsubsection{Modelling of Epitaxial Growth}

Simulations of epitaxial growth are of great scientific and technological interest. They can provide us with a fuller understanding of the evolution of the surface morphology during the growth process. They are a cost effective way of determining the sensitivity of a growth process to the relevant parameters. Additionally, the effect of significant changes in key parameters on the growth of an epitaxial layer, with respect to a given growth technique, can be quantified. Similarly, simulations allow ready testing of proposals for the improved control of a growth process. They also enable study of growth in regimes that are not readily reached in experiments. 


\subsubsection{Hybrid Models}

Modelling of epitaxial growth poses considerable difficulties - in particular the long time scale involved in the process and the importance of capturing enough atomistic detail, as discussed in Chapter 2.

In this thesis we develop a novel hybrid methodology to attack these problems. This methodology effectively increases the accuracy of Kinetic Monte Carlo (KMC) methods by incorporating fine grained Molecular Dynamics (MD) modelling in small specified regions. The KMC and MD methods are explained in Chapter 3. Alternatively it can be seen as increasing the practical size scale of Molecular Dynamics simulations by approximating large regions by a coarse grained computationally less demanding Kinetic Monte Carlo model, when this is suitable.

Stacking faults occur when separate parts of a layer of atoms differ in their registration with respect to the layers below. Parts of layers with different stacking are unable to mesh together, giving rise to grain boundaries where they meet. When stacking fault grain boundaries occur in the process of epitaxial growth, they reduce the quality of the grown material. The hybrid model developed in this thesis, is ideally suited to modelling stacking fault grain boundaries, to which it is applied in this work.

A number of other hybrid methodologies have been developed to try and address these issues. We note that Pomeroy et al. [20] have described a scheme for hybrid MD KMC. However, their scheme involved consecutive application of MD and KMC rather than the concurrent, spatially decomposed algorithm used here. Several other hybrid schemes have been developed for modeling epitaxial growth based on spatial decomposition, including an algorithm that couples the continuum BCF model to KMC [21, 22]. In principle, it would be possible to combine several such hybrid schemes with the method described here. 


\subsection{Chapter Overviews}

\subsubsection{Chapter 2: Epitaxial Growth}

As discussed in Chapter 2, a major issue in modelling epitaxial growth is the wide range of length and time scales that play a part in the phenomena. For modelling all aspects of epitaxial growth, neither of the atomistic methods, Molecular Dynamics or Kinetic Monte Carlo, is sufficient. MD cannot address the long time scales involved. KMC may not capture all the important atomic configurational changes. Continuum methods, such as Burton Caberera Frank cover the time scale but ignore important atomistic details. As a result hybrid models such as the model described in this thesis have been developed to address these issues.

\subsubsection{Chapter 3: Methodologies}

Chapter 3 describes the modelling methodologies used in the work. The Molecular Dynamics and Kinetic Monte Carlo simulation methods are discussed. Additional theory used in applying KMC to epitaxial growth simulations, i.e. Transition State Theory and the Nudged Elastic Band method, are also explained.

\subsubsection{Chapter 4: Molecular Dynamics}

In Chapter 4 we carry out Molecular Dynamics modelling to set a reference frame for our hybrid model. We show that Molecular Dynamics simulations using the Lennard Jones potential can model dynamic stacking fault grain boundaries in an epitaxial layer on a (111) surface. These simulations reproduce experimentally observed features, e.g. a tendency to form triangular shaped islands. The grain boundaries continually alter their shape and position. The grain boundaries migrate over the surface following a temperature dependent random walk. Over long time periods a small drift in grain boundary position is observed, in addition to the random diffusion. A pair of grain boundaries can meet and annihilate each 
other - eliminating the stacking fault. The key atomic process for grain boundary migration is shown to be the "kink flip" move.

\subsubsection{Chapter 5: Hybrid Modelling of Grain Boundaries}

Our hybrid MD KMC modelling methodology was developed and applied to the same problem studied with MD in Chapter 4. The hybrid methodology was used to follow the propagation of a stacking fault grain boundary between Face Centered Cubic (FCC) and Hexagonal Close Packing (HCP) islands nucleating on a FCC (111) surface.

This hybrid methodology combines Molecular Dynamics and Kinetic Monte Carlo methodologies within a single concurrent atomistic simulation. Different domains within this hybrid simulation are modelled with different methodologies (MD or $\mathrm{KMC}$ ). And as the simulation progresses the model applied to a given domain is changed (perhaps several times) as necessary, so that the stacking fault grain boundary is always modelled with MD, as it moves about the surface.

This method leads to a speed up over conventional MD, reducing the execution time by a factor approximately equal to the relative fraction of remaining MD atoms. We show that it is possible to reproduce grain boundary mobilities from full MD simulations with the domain decomposition method in the absence of KMC events - when the MD regions are sufficiently large with length $30 \sigma$ (where $\sigma$ is the length scale defined in section 3.2.6).

We have also used the method to study the effect of over layer adatoms on the mobility of the boundaries, demonstrating that boundaries can become trapped by adatom islands. These effects would have been difficult to capture in a conventional KMC simulation.

\subsubsection{Chapter 6: Model Enhancement}

In Chapter 6 a way to enhance the hybrid model by reducing wave reflections from the MD KMC interface is investigated. The waves considerd in this theis 
are lattice vibrations. It is shown that the reflection coefficient is a function of the Langevin damping parameter $\gamma$. It is proposed that by suitable tuning of $\gamma$ in the region adjacent to the rigid $\mathrm{KMC}$ atoms, the reflections of waves back into the MD domain can be minimised. This is not tested but left for future development.

\subsection{Summary}

This thesis describes a novel hybrid computational methodology in which the Molecular Dynamics and Kinetic Monte Carlo methods are concurrently combined.The hybrid methodology has been developed to model features of epitaxial growth which are unfeasible to treat with either Molecular Dynamics or Kinetic Monte Carlo alone. The dynamics of epitaxial stacking fault grain boundaries have been elucidated using the hybrid methodology. Methods for enhancing the hybrid model are discussed. Any situation where the problem can be decomposed into distinct domains of fine scale and coarse scale modelling, is potentially suitable for treatment with a hybrid model of this design. 


\section{Chapter 2}

\section{Epitaxial Growth}




\subsection{Introduction}

This chapter gives an introduction to epitaxial growth and its technological role. Further information on these topics can be found in $[23,24]$. We then discuss methods of modelling epitaxial processes and the use of hybrid methodologies.

\subsection{Epitaxial Growth}

Epitaxial growth [1] refers to the growth of additional crystalline material on top of an existing crystal substrate, where the additional material grows in alignment and registration with the substrate. The growth results from controlled deposition of adatoms (adsorbed atoms that diffuse on the suface) onto the substrate. When the additional material forms atomic layers on the substrate, the result is a crystal film coherently orientated on the crystal substrate - an epitaxial thin film. The growing material and the substrate may be composed of the same or distinct materials. When the two materials are the same we have ordinary crystal growth. If the substrate is a single crystal the epitaxial layer will be also, otherwise a polycrystalline layer will form [23]. At very high deposition rates amorphous islands may form.

\subsection{Technological Importance}

Epitaxial growth is the most cost effective method of producing high quality crystals of many technologically important semiconductor materials, e.g. Si [24], SiGe [25], GaN [26] and GaAs [27].

The formation of epitaxial thin films is fundamental to the manufacture of many electronic and optoelectronic devices, because these devices are constructed from layers of different materials and different doping states of the same material. Light emitting diodes and semiconductor lasers are based on heteroepitaxial films [28]. 
For electronic devices $\mathrm{Si}$ is the typical substrate. In device fabrication the deposit materials may be elementary semiconductors, e.g. Si or Ge [29]. More complicated deposits using two element compounds, such as GaAs [30] or CdTe [31], may be used. Ternary compounds such as $\operatorname{In}_{x} \mathrm{Ga}_{1-x}$ As [32] may also be deposited.

When manufacturing transistors in semiconductor devices, an initial stage is the homoepitaxy of Si on Si. This is done because the epitaxial Si layer is usually freer of defects than the underlying Si substrate and the epitaxial layer can be doped independently of the substrate [24].

\subsubsection{Applications of epitaxy}

There are many applications of epitaxy in the manufacture of semiconductor and thin film devices.

- Development of new wide-band gap optoelectronic devices, e.g. LEDs and lasers based on GaInN [33].

- Fabrication of high speed high frequency communications devices based on $\mathrm{Ge}_{x} \mathrm{Si}_{1-x}$ [34] and compound semiconductor materials [35].

- The fabrication of multilayer heterojunction composites, including quantum wells and superlattices. By engineering bandgap structures some remarkable nanoscale quantum electronic and optical devices have emerged, e.g. quantum well lasers, high electron mobility transistors, quantum cascade lasers and quantum hall effect devices [36, 37, 38].

- Deposition of orientated electroceramic films and multilayers for varied purposes, e.g. high temperature superconductors, magnetic garnets for magnetooptical devices and ferroelectric films for memory applications [39]. 


\subsection{Features of Epitaxial Growth}

\subsubsection{Homo-and Hetero-Epitaxy}

A simple definition of homoepitaxy is that it occurs when the deposit material and the substrate are the same, e.g. deposition of Ge onto Ge [40]. Heteroepitaxy is then the deposition of a material different to the substrate, e.g. deposition of (AlGa)As onto GaAs [41]. Heteroepitaxy is the more common situation technologically.

The difficulty of achieving heteroepitaxy between given deposit and substrate materials depends on:

- the difference between the crystal lattice constants of the materials, i.e. the lattice mismatch [42], and

- the balance between the cohesive energies within each of the materials and the adhesion energy at the material interface [24].

The lattice mismatch in heteroepitaxy will vary depending on the material used in the substrate and the deposit; typically the mismatch is less than $7 \%$, as larger mismatches can lead to textured or polycrystalline films [43].

\subsubsection{Deposition Methods}

A number of methods are available to produce epitaxial deposits of one material upon another. The methods can be broadly classified into either physical or chemical.

Physical vapour deposition (PVD) [44] methods rely on heat induced evaporation or sublimation in vacuum of the material to be deposited. The hot deposit material then travels in a controlled fashion through the vacuum to the substrate, where epitaxial growth occurs. Evaporation deposition has a long history and is widely used in producing optical thin films, wide area coatings and epitaxial thin films. An alternative to evaporation is sputtering [45]. In sputtering it is the impact 
of gaseous ions with the material to deposited, rather than heating, which causes atoms to be ejected from the source of the deposit material into the vacuum. Sputtering is commonly employed when alloy films are required.

In chemical vapour deposition (CVD) [46], a volatile compound of the material to be deposited is allowed to decompose on the hot substrate producing a nonvolatile solid. This results in the epitaxial growth of the deposit material, while volatile by-products leave the surface. Chemical vapour deposition methods are used for depositing nonmetallic hard coatings, dielectric films and high quality epitaxial (single crystal) semiconductor films.

Some approaches employ both physical and chemical methods [47]. In molecular beam epitaxy (MBE) [48, 49], beams of different elements can be directed at the substrate so the deposit grows one atomic layer at a time. For example, GaAs layers can be deposited by repeatedly applying a layer of $\mathrm{Ga}$ and then a layer of As, which then combine chemically to form GaAs. The beams may be produced by heating or by chemical reactions.

\subsubsection{Surface analysis}

The process of epitaxial growth and the development of the epitaxial films can be monitored and analysed using a variety of techniques. The techniques generally utilise either diffraction or real space imagery. A commonly used diffraction based technique is reflection high energy electron diffraction (RHEED) [50], while scanning tunneling microscopy (STM) [51] is a widely used real imaging technique.

The RHEED technique records the diffraction of high energy electrons that have been directed towards the surface at a glancing angle. RHEED measurements allow monitoring of the crystallinity of the growing film [52].

In STM the tunnelling current between the atom scale tip and the surface is measured. By scanning the tip over the surface, information related to the charge density at each scan point is obtained. This information provides near atomic resolution images of the detailed morphology of an epitaxial film during the growth of the film $[53,54]$. STM is not suitable for production line monitoring as it is too 
time consuming.

\subsubsection{Epitaxial Growth Modes}

There are three well known epitaxial growth modes [55]:

- (a) Volmer-Weber (VM) or island growth [56],

- (b) Frank-van der Merwe (FM) [57] or layer-by-layer growth, and

- (c) Stranski-Krastanov (SK) [58] or layer-by-layer growth followed by formation of islands.

These growth modes are illustrated in Figure 2.1.
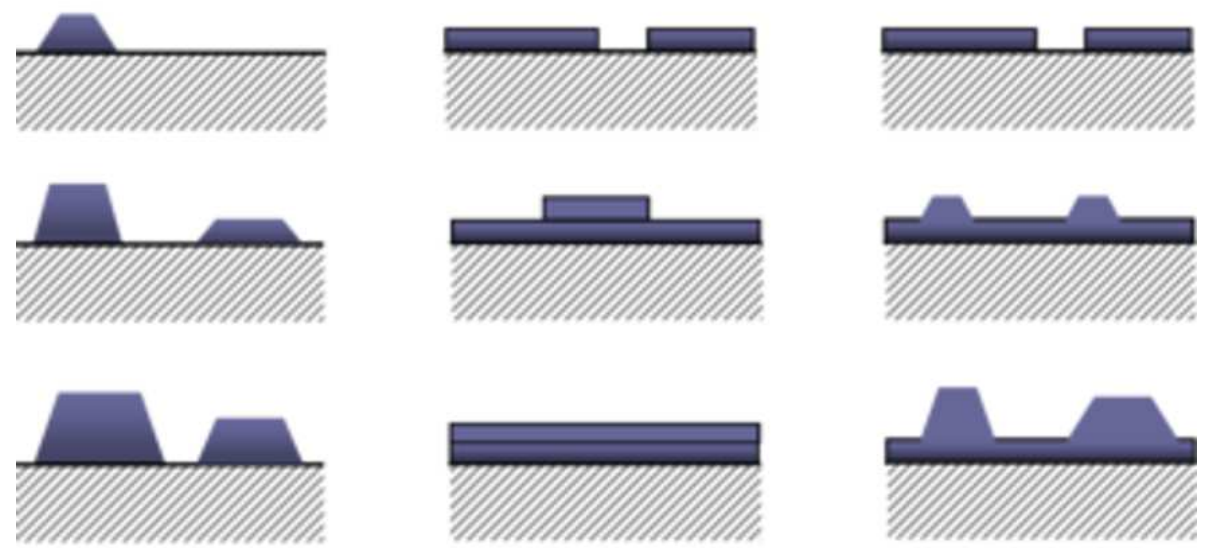

(a)

(b)

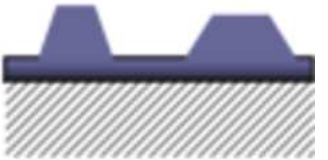

(c)

Figure 2.1: Snapshots (cross-section view) of epitaxial growth in the three growth modes: (a) Volmer-Weber island growth, (b) Frank-van der Merwe layer-by-layer growth and (c) Stranski-Krastanov layer-by-layer growth followed by islands. Elapsed time and surface layer coverage increase progressively down the columns.

Which growth mode occurs depends on the wetting of the substrate by the deposited material, and the lattice mismatch between the deposit and the substrate $[23,59]$. 
When the deposit does not fully wet the substrate, VM mode occurs. In the case of full wetting and a small lattice mismatch between deposit and substrate, FM mode is predominant. If there is full wetting but a significant lattice mismatch SK mode ensues.

FM growth is favoured when the sum of the free energy of the vacuum deposit interface and the free energy of the deposit substrate interface is less than the free energy of the vacuum substrate interface. Then wetting of the substrate by the deposit is energetically favourable.

When the free energy of the vacuum substrate interface is less than the sum of the other two free energies, formation of a deposit layer over the whole substrate is disfavoured as it will increase the free energy of the system. In such cases VM growth of isolated deposit islands on the substrate is favoured.

The SK growth mode is a response to the strain caused by the difference in lattice constants between the deposit and the substrate. Initially a wetting layer epitaxial film up to a few monolayers thick is formed. This film is strained to match the lattice constant of the underlying substrate, with the strain increasing as the film thickens. Beyond a certain thickness the strain can be relaxed by misfit dislocations resulting in the formation of separate islands on top of the film.

The following trends in growth mode generally hold [60]:

- FM growth is exhibited at low substrate temperatures, with SK or VM growth at high substrate temperatures.

- The higher the rate of deposition, the more layer-like the resulting growth and vice versa.

- With a larger lattice mismatch the growth is more island-like.

- Denser packing of the substrate facet produces more layer-like growth.

\subsubsection{Layer Growth}

The FM growth mode can be understood in terms of the following atomic scale processes. Adatoms are deposited onto the substrate and diffuse across it. Adatoms 
may meet and bind with each other or they may attach to screw dislocations. In either case, small monolayer islands are formed. Additional adatoms attach to the edges of the island causing them to grow, until eventually a monolayer is formed. This process may then be repeated on top of the newly formed monolayer, creating over time another monolayer. In this way an epitaxial thin film can be grown a monolayer at a time.

At any point there may be many partly completed monolayers stacked on top of each other in a series of steps. Adatoms deposited on the terraces between the steps diffuse across the terraces and attach to the step edges. The adatoms reaching a step edge may diffuse along the step edge before becoming attached, or they may diffuse away again. An adatom is more likely to be successfully incorporated into a step edge at kink sites, where there are more neighbouring atoms, and so more bonds to hold the adatom in place. When an adatom attaches to a flat section of a step edge, a kink site is formed on either side of the adatom's edge position. Once an adatom attaches to an existing step edge kink site, the attached adatom creates a new kink site one atom spacing further along the step edge. As a result of the kink sites on the step edge capturing adatoms, and then moving along the step edge, the step edge will eventually have an adatom attached at all lattice sites along its length. At this stage the position of the step has moved by one row of atoms, enlarging the terrace by one atomic row. In this way the positions of the step edges flow across the surface and the terraces eventually grow to form full monolayers. When an adatom reaches a step edge it may also go up or down over the step rather than attach to it. The Schwoebel barrier [61] is an energy barrier that inhibits diffusing atoms on terraces from stepping down to lower levels. An Schwoebel barrier can lead to a coarsening of the evolving surface morphology. In the presence of a Schwoebel barrier, growth by step flow is stable only if the surface has sufficient density of steps [62]. 


\subsection{Modelling Epitaxial Growth}

A major issue in modelling epitaxial growth is the wide range of length and time scales that play a part in the phenomenon. For technological purposes, a complete epitaxial film covering a $\mathrm{Si}$ wafer has an area in the order of $0.02 \mathrm{~m}^{2}$ and the time for the growth of a monolayer can be in the order of $1 \mathrm{~s}-1 \min$ [63]. To fully model the properties and morphology of such epitaxial films requires taking account of the movements of adatoms on surfaces, their interactions, and the resulting layer formation and subsequent growth to macroscopic size. There is presently no single modelling methodology that can accurately encompass the length and size scales involved. The usual approach to handling this issue is to use a combination of different metholodgies, each of which covers a part of the time and length scales. Information produced by the methodology operating at a fine space and time scale, plus any parameters determined by fit to experiment, must be passed up to the methodology operating at the next coarse scale. For example, detailed information on atom positions from a Molecular Dynamics model would be simplified to an occupied lattice site in a Kinetic Monte Carlo model.

\subsubsection{Atomistic Modelling Methodologies}

Atomistic methods take explicit account of the atoms that compose the material being modelled. Molecular Dynamics and Kinetic Monte Carlo are two widely used atomistic methods. These methods are discussed in more detail in Chapter 3.

In MD the vibration of the atoms, and subsequent larger atomic motions occuring on longer time scales, are derived from the forces acting on the atoms. The forces in turn are typically derived from a potential. Potentials may be derived empirically or from more detailed quantum mechanical calculations. In theory MD is a very suitable method for modelling epitaxial growth. In practice there are two problems with MD that limit its usefulness. Firstly, the form of the potential for a particular system is not always easy to determine. Secondly, MD requires the use of a small time step to maintain the accuracy of a simulation, while the force calculation tends to scale superlinearly with the number of atoms [64]. Thus when 
large numbers of atoms are modelled, simulation of long time scales requires long computer runs. Therefore in practice MD simulations are limited to simulating nanosecond time scales.

The KMC method forgoes the detailed atomic dynamics that are simulated by $\mathrm{MD}$, thereby avoiding the need to calculate interatomic forces. Instead, only the rate limiting events are included on average, based on effective rates of occurrence. As a consequence $\mathrm{KMC}$ is computationally cheaper than $\mathrm{MD}$, and so it can simulate a wider range of time and space scales. The main difficulty with KMC is that the interatomic events to be modelled must typically be identified beforehand [65], and their rates determined and coded into the model. For example, when KMC is used to simulate FM epitaxial growth, events such as adatom hopping, adatom attachment to and detachment from step edges, and formation of dimers and trimers would need to be included in the model with appropriate rates.

\subsubsection{Continuum Modelling Methodologies}

The Burton Caberera Frank (BCF) [66] method models the growth of epitaxial layers as the result of the flow of steps across the surface. It does not explicitly simulate the adatom motions or the atomistic detail of the step edges. Instead the step edge is modeled as a moving boundary, and adatoms on the terraces above and below the step are represented by adatom densities.

The main assumptions made in the BCF method are: steady state conditions, a series of equally spaced straight steps, a single atomic species, adatom diffusion occurring much faster than step flow, equal probability for a step to capture adatoms from the upper and lower terrace and low adatom density on terraces.

The BCF model describes the adatom density evolution by Eq. (2.1), which equates the change in adatom density to the combined effect of adatom surface diffusion less adatom evaporation plus adatom deposition

$$
\frac{\partial \rho(x, t)}{\partial t}=D \frac{\partial^{2} \rho(x, t)}{\partial x^{2}}-\frac{1}{\tau} \rho(x, t)+F,
$$

where $x$ is the direction perpendicular to the step, $t$ is time, $\rho(x, t)$ the adatom 
density on the terrace, $D$ the adatom surface diffusion constant, $\frac{1}{\tau}$ the probability of adatom evaporation and $F$ the adatom deposition rate. Assuming that the emission and capture of adatoms at steps is rapid, the boundary condition on the adatom density at the steps is $\rho(x)=\rho_{0}$, where $\rho_{0}$ is the equilibrium adatom density for the given temperature $\mathrm{T}$ (assuming an infinite surface at equilibrium with its saturating vapour). The formula for $v$, the velocity of a step separating two terraces of respective widths $w_{1}$ and $w_{2}$, is derived from Eq. (2.1) giving [67]:

$$
v=D\left(F \tau-\rho_{0}\right) k\left[\tanh \left(\frac{k W_{1}}{2}\right)+\tanh \left(\frac{k W_{2}}{2}\right)\right]
$$

where $k=\sqrt{\frac{1}{D \tau}}$.

Extensions of BCF have been developed that relax some of its assumptions. For example Ghez [68] allows for near equilibrium adatom concentration at steps and for fast step flow, while modelling of multiple species has been undertaken by Mandel [69], and Caflisch et al [70] treat high deposition far from equilibrium conditions.

The BCF method operates at a coarser level of description than KMC. BCF does not model features such as kinks in the step edge, interactions among adatoms and the Schwoebel barrier [61], which are important in many cases.

A further development of the BCF method is Island Dynamics (ID) [71],[72]. ID allows for growth on the terraces. ID models epitaxial growth resulting from the nucleation, growth and subsequent coalescence (or otherwise) of islands. In ID the island edges are the moving boundaries of interest. The ID equation for adatom density change is based on Eq. (2.1), with an additional term representing loss of adatoms due to island nucleation. ID handles much higher deposition rates than BCF. The level set method [73, 74], is a numerical method used in ID to track the motion of the island edges [71]. The phase field method [75], is another front tracking technique that can be used for this purpose. Different variants of ID are distinguished by different formulas for the boundary conditions and the velocity.

Other continuum methods using even coarser representations of epitaxial growth have been developed. These methods typically follow the evolution of the height profile of the epitaxial surface through a smooth height function $h(x, y, t)$ [76]. 
For example, the growth of the height of a surface over time can be modelled using the Khardar-Parisi-Zhang (KPZ) equation [77],

$$
\frac{\partial h(x, t)}{\partial t}=s \nabla^{2} h(x, t)+\frac{\lambda}{2}(\nabla h(x, t))^{2}+\eta(x, t) .
$$

The first term on the right hand side of the KPZ equation models the smoothing of the shape of the surface due to the surface tension, $s$. This term redistributes the irregularities of the surface without changing the average height. The second term is nonlinear, and accounts for lateral growth which occurs along the local normal to the surface. The third term on the right hand side, is uncorrelated random noise with mean zero, which reflects random fluctuations in the deposition process. The KPZ equation can be extended to model the change in height of a two dimensional surface $h(x, y, t)$, by separating each of the terms into $x$ and $y$ terms with distinct coefficients, and replacing $\nabla$ by the corresponding partial derivative. This extended KPZ equation can allow for the effects of surface anisotropy introduced by the presences of steps, e.g. differential growth parallel and perpendicular to step edges.

In this hierarchy of methods, detailed representation has been progressively reduced in order to access larger time and size scales. Particular methods may be well suited to a particular level of description, but no single method is capable of fully capturing epitaxial growth.

\subsection{Hybrid Methods}

For modelling all aspects of epitaxial growth, neither of the atomistic methods, MD or KMC, is sufficient. MD cannot practically address the long time scales and large numbers of atoms involved. KMC may not capture all the important atomic configurational changes. Continuum methods, such as BCF, cover the time scale but ignore important atomistic details.

An approach to handling these issues is to combine distinct methods into a hybrid method. An example is the atomistic-continuum method of Schulze [21]. Lam and Valachos [78] employ a hybrid scheme combining a continuum model 
and Monte Carlo to study the transition from step flow to two-dimensional island nucleation. Kundin and Emmerich [79], have investigated the same transition with a hybrid algorithm employing a phase field model and KMC.

In this thesis we develop a hybrid method which concurrently combines MD and KMC. This hybrid method retains the detail and atomistic dynamics of MD, but has a reduced computational cost compared to MD with the same number of atoms. The cost reduction is the result of limiting the MD force calculation to a subset of atoms, with KMC being applied to the balance of the atoms. The division of atoms into MD and KMC subsets is dynamic and can change during the course of a simulation. This method has been used to study stacking fault grain boundaries in epitaxial layers [80]. 


\section{Chapter 3}

\section{Modelling Methodologies}




\subsection{Introduction}

This Chapter describes the modelling methodologies used in this work. This thesis presents a novel hybrid of the MD and KMC methodologies. Simulations using the hybrid methodology will be assessed for consistency with comparable MD simulations. This Chapter discusses each of MD and KMC as they are applied individually.

\subsection{Molecular Dynamics}

Molecular Dynamics is a methodology for simulating the behaviour of a system of discrete particles. This behaviour emerges as a result of the particles interacting in a specified manner. Consequently the nature of the interaction assumed between the particles is a key part of the MD methodology. These interactions between the particles determine interparticle forces. As a result of these forces the positions and velocities of the particles are changed.

The MD simulation evolves the configuration of the system through a sequence of discrete time steps by carrying out the following computations at each time step in the simulation:

- calculate forces based on differentiating a potential energy function applied to current positions

- update positions and velocities based on current forces using a discrete approximation to Newton's equations.

\subsubsection{Applications of MD}

Here we provide an overview of MD, with emphasis on those aspects that are most relevant to this work. MD simulations have been used in a range of applications:

- In materials science: crack propagation [81], crystal growth [82], bulk and 
surface diffusion [83], thermodynamic studies (determination of phase diagrams) [84].

- In chemistry: molecular reconfigurations [85], catalysis [86].

- In molecular biology: structure and behaviour of biomolecules [87], cell membrane mechanics [88], studies of DNA [89].

- In nanoscience: studies of nanoclusters [90], nano mechanics and tribology [91], nanoscale features [92].

In this work, MD is used in simulation studies of aspects of epitaxial growth. In particular, the behaviour of surface grain boundaries has been studied using MD alone and as part of a hybrid methodology.

\subsubsection{Equations of Motion}

The prototypical MD simulation involves numerically integrating Newton's equations of motion 3.1, for the $\mathrm{N}$ particles which comprise the system:

$$
m_{i} \frac{d^{2} \mathbf{r}_{\mathbf{i}}}{d t^{2}}=\mathbf{F}_{\mathbf{i}}\left(\mathbf{r}_{1}, \ldots, \mathbf{r}_{\mathbf{N}}\right) \quad \mathrm{i}=1, \ldots, \mathrm{N} .
$$

In this work the $\mathrm{N}$ particles are (idealised) atoms, with $m_{i}$ their masses, $\mathbf{r}_{\mathbf{i}}$ their position vectors, and $\mathbf{F}_{\mathbf{i}}$ the forces acting on them due to the interactions with the other particles in the system.

Typically, a potential function $V$ is specified at the start of the simulation and the forces (at each time) are determined from the negative gradient of the potential (at that time):

$$
\mathbf{F}_{\mathbf{i}}\left(\mathbf{r}_{1}, \ldots, \mathbf{r}_{\mathbf{N}}\right)=-\frac{d V\left(\mathbf{r}_{1}, \ldots, \mathbf{r}_{\mathbf{N}}\right)}{d \mathbf{r}_{\mathbf{i}}}
$$

Such potential functions are determined semi-empirically or from approximate quantum mechanical calculations. At each discrete time step in the simulation, the potential energy of the system is determined by applying the potential function to the atoms in their current geometric configuration. 
A system obeying Newton's Equation of Motion will conserve its total energy $\mathrm{E}$ at all times i.e. $\frac{d \mathrm{E}}{d t}=0$. However, the energy may well vary between potential and kinetic forms while maintaining the fixed total. When there is no chemical bonding between the atoms, it is sufficient to calculate the potential and hence the force on an atom, from its pairwise interactions with the other atoms, based on the radial distance between the atoms:

$$
V\left(\mathbf{r}_{\mathbf{i}}\right)=\sum_{j \neq i}^{N} V\left(r_{i j}\right) .
$$

Here the radial distance between atom $\mathrm{i}$ and atom $\mathrm{j}$ is: $r_{i j}=\left|\mathbf{r}_{\mathbf{i j}}\right|$ where $\mathbf{r}_{\mathbf{i j}}=$ $\mathbf{r}_{\mathbf{j}}-\mathbf{r}_{\mathbf{i}}$. The aggregate force experienced by an atom (at a given time) is the sum of the forces produced by each of its pairwise interactions with the other atoms in the system (at that time) and acts along the line between the pair of atoms. The force on atom $i$ from all the other atoms can be derived by expanding the positions

$$
\mathbf{F}_{\mathbf{i}}=\sum_{j \neq i}^{N}-\frac{d V\left(r_{i j}\right)}{d r_{i j}} \hat{\mathbf{r}}_{\mathbf{i j}}
$$

where $\hat{\mathbf{r}}_{\mathrm{ij}}$ is the unit vector from atom $\mathrm{i}$ to atom $\mathrm{j}$. Note that once the force on atom $\mathrm{i}$ due to atom $\mathrm{j}$ has been determined, the force on atom $\mathrm{j}$ from atom $\mathrm{i}$ is simply the negative of this force, by Newton's third law.

\subsubsection{Time Integration}

The integration routine numerically integrates the equations of motion over a simulation time step, advancing the positions and velocities of the atoms by a time step. Note that the positions and velocities may not be at the same time point, e.g. the velocity may be one step behind the position. Many integration formulae can derived by expanding the positions and velocities at time $t$ with time step $\delta t$, using Taylor series

$$
\mathbf{r}_{\mathbf{i}}(t+\delta t)=\mathbf{r}_{\mathbf{i}}(t)+\mathbf{v}_{\mathbf{i}}(t) \delta t+\frac{1}{2} \mathbf{a}_{\mathbf{i}}(t) \delta t^{2}+O\left(\delta t^{3}\right)
$$




$$
\mathbf{v}_{\mathbf{i}}(t+\delta t)=\mathbf{v}_{\mathbf{i}}(t)+\frac{1}{2} \mathbf{a}_{\mathbf{i}}(t) \delta t+O\left(\delta t^{2}\right)
$$

where $\mathbf{r}_{\mathbf{i}}, \mathbf{V}_{\mathbf{i}}$ and $\mathbf{a}_{\mathbf{i}}$ are the position, velocity and acceleration respectively, of atom i. The acceleration is calculated from the force

$$
\mathbf{a}_{\mathbf{i}}(t)=\mathbf{F}_{\mathbf{i}}(t) / m_{\mathbf{i}}
$$

Combinations of expansions such as these can then be used to produce integration formulae. For example, consider the Taylor series for a backward time step in position

$$
\mathbf{r}_{\mathbf{i}}(t-\delta t)=\mathbf{r}_{\mathbf{i}}(t)-\mathbf{v}_{\mathbf{i}}(t) \delta t+\frac{1}{2} \mathbf{a}_{\mathbf{i}}(t) \delta t^{2}-O\left(\delta t^{3}\right) .
$$

Adding Eq. (3.2) and Eq. (3.4) and rearranging gives the following equation for $\mathbf{r}_{\mathbf{i}}(t+\delta t)$

$$
\mathbf{r}_{\mathbf{i}}(t+\delta t)=2 \mathbf{r}_{\mathbf{i}}(t)-\mathbf{r}_{\mathbf{i}}(t-\delta t)+\mathbf{a}_{\mathbf{i}}(t) \delta t^{2} .
$$

Similarly, subtracting Eq. (3.2) and Eq. (3.4) and rearranging gives

$$
\mathbf{v}_{\mathbf{i}}(t)=\frac{\mathbf{r}_{\mathbf{i}}(t+\delta t)-\mathbf{r}_{\mathbf{i}}(t-\delta t)}{2 \delta t} .
$$

Note that in this case $\mathbf{v}_{\mathbf{i}}(t)$ can only be computed once $\mathbf{r}_{\mathbf{i}}(t+\delta t)$ is known. These equations, Eq. (3.5) and Eq. (3.6), define the Verlet integration formula [64].

The advantages of the Verlet formula when compared to more complicated formulas such as the Gear predictor-corrector [64] are: no systematic drift in total energy of the system, good stability when time step is small enough, only one force call per time step, and ease of implementation.

\subsubsection{Periodic Boundary Conditions}

MD simulations are often designed to provide information about macroscopic sized systems, composed of approximately $10^{23}$ atoms. In the 1990 's computers capable of $10^{9}$ floating point operations per second (FLOPS) became available, enabling the MD simulation of millions of atoms. Computer power has continued to grow since then. Currently the most powerful supercomputers can perform $10^{15}$ FLOPS and state of the art MD simulations of $10^{12}$ atoms are possible $[93,94]$. 
However very few groups have the resources to carry out such large simulations. In practice, MD simulations commonly use a few thousand atoms. Creating a small subset of the macroscopic system introduces artificial surfaces. In addition, the proportion of surface atoms in such a simulation is much larger than the proportion of surface atoms in the macroscopic system. These features can distort the simulation, because the surface atoms may have missing bonds, giving them an artificially large potential energy. These issues are usually dealt with by the use of periodic boundary conditions (PBC), which mimic the effect of being part of the bulk and effectively eliminate the artificially introduced surfaces, while still limiting the length scale on which properties can be calculated. PBC involves treating the set of atoms in the simulation as being surrounded by multiple images of itself. In this way any missing bonds can be replaced by bonding with an atom in one of the images. When calculating pairwise interactions between atoms, the closer of the direct position of the atom and its nearest image position is used.

\subsubsection{Temperature Control}

The MD methodology described up to this point, in which Newton's equations of motion are integrated, is only the simplest case. In such cases a constant energy ensemble is simulated. The total energy of the system remains constant throughout the simulation while its individual components, potential energy and kinetic energy, vary. At thermal equilibrium the equipartition theorem [95] relates the average kinetic energy to the temperature $T$ of the system during the simulation via: $<\frac{1}{2} m v^{2}>=\frac{3}{2} k_{B} T$, where $k_{B}$ is the Boltzmann constant.

In many situations of interest the system under study is in intimate thermal contact with a much larger environment and thus the system's temperature is maintained at the temperature of the environment. To simulate such systems using MD the simplest case equations of motion need to be modified so that the temperature of the system is controlled. Such modifications are known as thermostats.

MD thermostats may be divided into two classes - deterministic and stochastic. The simplest deterministic thermostat is velocity scaling, where at each time 
step the velocities of the atoms are scaled to maintain a predetermined kinetic energy, and hence temperature. This has the disadvantage of eliminating the natural fluctuations of the temperature about its average value.

The Langevin thermostat [96] is in the stochastic class. It involves the addition of both a "frictional" damping force, and random jostling of the motion of the atoms, to the previously discussed equation of motion Eq. (3.1). The revised equation of motion incorporating a Langevin thermostat, for atom $\mathbf{i}$ with mass $m_{\mathbf{i}}$ and position vector $\mathbf{r}_{\mathbf{i}}$ is:

$$
m_{\mathbf{i}} \frac{\mathrm{d}^{2} \mathbf{r}_{\mathbf{i}}}{\mathrm{d} t^{2}}=-\nabla V\left(\mathbf{r}_{\mathbf{i}}\right)-\gamma \frac{\mathrm{d} \mathbf{r}_{\mathbf{i}}}{\mathrm{d} t}+\mathbf{R}_{\mathbf{i}}(t)
$$

There are now three forces involved. Firstly, the force from all pairs of atoms, secondly, a damping term $\gamma$, proportional to the velocity of the atom, and thirdly, a random force $\mathbf{R}_{\mathbf{i}}$. The random force is uncorrelated across time and across atoms. The combination of the damping term and the random term simulates the effect of being in contact with a heat bath. In respect of each coordinate direction, the fluctuation-dissipation theorem $[97,98]$ relates the damping $\gamma$, the random force $\mathbf{R}_{\mathbf{i}}$ and the temperature $T$ by:

$$
<\mathbf{R}_{\mathbf{i}}\left(t^{\prime}\right), \mathbf{R}_{\mathbf{j}}(t)>=2 m_{\mathbf{i}} \gamma k_{B} T \delta\left(t^{\prime}-t\right) \delta_{\mathbf{i j}}
$$

This ensures that a constant chosen average temperature is maintained due to a balance between the heating effect of $\mathbf{R}$ and the cooling effect of $\gamma$. In this work the random fluctuations were uniformly distributed on $(-\sqrt{2 \gamma T}, \sqrt{2 \gamma T})$, where we have used $m=1$ and $k_{B}=1$ in Eq. (3.8), as discussed in section 3.2.6. The default value used for the damping parameter was $\gamma=1.0$ [20].

To implement this revised equation of motion the Verlet integration formula is modified to include the damping and random forces.

\subsubsection{The Lennard-Jones Potential}

The Lennard-Jones potential [99] was developed to model systems in which the dominant (or only) interaction between the particles is the van der Waals force. 
This potential $V_{L J}(r)$ has two parameters $\epsilon$ and $\sigma$, and depends on the interatom radial distance $r$ as follows:

$$
V_{L J}(r)=4 \epsilon\left[\frac{\sigma^{12}}{r^{12}}-\frac{\sigma^{6}}{r^{6}}\right]
$$

The strength of the interaction (the depth of the potential well) is given by $\epsilon$, while $\sigma$ gives a length scale. The largest negative value of the potential (depth of the potential well) is $-\epsilon$, which is the potential's minimum at $r=2^{\frac{1}{6}} \sigma$. When the interatom distance $r$ is less than this value, the potential gives rise to a strong repulsive force between particles, whereas for interatom distances greater than this, a weak attractive force arises.

The repulsive force at short distance, that arises from the first term $\frac{1}{r^{12}}$, models the repulsion due to overlapping full electron orbitals. And the attractive force from the second term $-\frac{1}{r^{6}}$, which predominates at longer distances, models the attractive van der Waals dispersion forces. Thus the Lennard-Jones potential provides a very good model for weakly bonded closed-shell elements such as Neon, Argon and Krypton, when the parameters are tuned to the physical properties of these materials [100]. This potential was initially proposed for liquid Argon [101].

The Lennard-Jones potential is not sufficient for accurate determination of material and thermodynamic properties when modelling open shell systems. In open shell systems with strong localised and directional bonds, the weaker van der Waals forces only play a minor role. However the general features exhibited by the Lennard-Jones potential, i.e. short range repulsion, an equilibrium spacing and longer range attraction, are common to a wide range of systems. These features combined with the simplicity of the Lennard-Jones potential have resulted in its widespread use as a general first approximation in MD. In this work the LennardJones potential has been adopted for these reasons.

Mixtures of Lennard-Jones materials can be treated by using the Bertholt combination rules to produce suitable values of $\epsilon$ and $\sigma$. That is for two elements labeled by $a$ and $b$, the combination rules give parameter values for the mixture $a b$ as:

$$
\sigma_{a b}=\frac{1}{2}\left(\sigma_{a}+\sigma_{b}\right)
$$




$$
\epsilon_{a b}=\sqrt{\epsilon_{a} \epsilon_{b}} .
$$

As an alternative, the attractive part of the potential may be varied.

In this work, dimensionless units known as Lennard-Jones reduced units have been employed. These reduced units are constructed by choosing $m$ as a standard unit of mass, $\sigma$ as a standard unit of length and $\epsilon$ as a standard unit of energy. All other dimensioned quantities are then expressed in terms of these standard units and become dimensionless numeric values. Thus all distances are in terms of $\sigma$, all energies in terms of $\epsilon$, temperature is in terms of $\epsilon / k_{B}$ and time is in terms of $\sigma\left(\frac{m}{\epsilon}\right)^{\frac{1}{2}}$. We denote this Lennard-Jones reduced time unit by $t$. The parameters in the Lennard-Jones potential both now have numeric value 1 which simplifies subsequent formulae and calculations. Also, in this work all atoms have the same mass $m$ with reduced numeric value 1 . In these reduced units $k_{B}$ is also set to 1. The main advantage of using such dimensionless units in simlation work is that the results from a single model can be scaled to a whole class of systems. In a Lennard-Jones material, melting occurs at the reduced temperature triple point $T=0.694$. For example in the case of Argon, $\epsilon=0.0104 \mathrm{eV}$ [102] implies that the unit of reduced temperature (i.e. $T=1.0 \epsilon / k_{B}$ ) corresponds to $121 \mathrm{~K}$, and so $T=0.694$ corresponds to $84 \mathrm{~K}$, the melting point of Argon [102]. In this work reduced temperatures in the range $T=0.35$ to $T=0.50$ are used. These correspond to $51 \%$ and $72 \%$ of the melting temperature respectively. Comparing to Iridium with a melting point of $2719 \mathrm{~K}, T=0.50$ corresponds to $1959 \mathrm{~K}$. Over these temperature ranges the simulated slab of material remains solid, but there is sufficiant thermal energy for atoms to hop between adjacent lattice sites frequently enough that a simulation run exhibiting significant grain boundary motion could be completed in less than 8 hours of wall clock time.

The Lennard-Jones potential is a member of the set of potentials known as pair potentials. These potentials get their name from the fact that they are based solely on interactions between pairs of particles. The local environment (i.e. the number and the configuration of neighbouring particles) is not explicitly taken into consideration. 
Another popular pair potential is the Morse potential [103, 104]

$$
V_{\text {Morse }}(r)=D\left[1-\exp \left(-\beta\left(r-r_{0}\right)\right)\right]^{2} \text {. }
$$

The parameter $r_{0}$ stands for the nearest neighbour lattice spacing, and $D$ and $\beta$ are additional fitting parameters. The Morse potential is computationally more expensive than the Lennard-Jones potential, but is more realistic for many materials.

\subsubsection{The Embedded Atom Method}

In many situations the local environment is very important in determining the potential energy of a system of particles. For example, at a surface of a crystal, the atomic bonds may have different properties than in the bulk. Pair potentials do not capture this effect. In metals, the properties of chemical bonds between pairs of atoms can depend strongly on the local environment. This is due to quantum mechanical effects that describe the influence of the electron gas. To more accurately model such situations, so called multibody potentials were developed. In such potentials the potential energy between two particles is no longer simply a function of the distance between them, but also a function of the positions of all other particles in the vicinity, i.e. a function of the local environment. In the case of metals, the interactions of atoms can be quite accurately described using potentials based on the embedded atom method (EAM) [105, 106, 107]. The EAM potential features a contribution by a pair potential (two-body) term to capture the basic repulsion and attraction of atoms, in conjunction with a multibody term that accounts for the local electronic environment of the atom.

An EAM potential for metals is typically given in the form

$$
V_{E A M}(i)=\sum_{j=1}^{N_{i}} \phi\left(r_{i j}\right)+f\left(\rho_{i}\right),
$$

where $\phi$ is the pair potential, $\rho_{i}$ is the local electron density and $f$ is the embedding function. The electron density $\rho_{i}$ depends on the local environment of atom $i$, and 
the embedding function $f$ describes how the energy of an atom depends on the local electron density. The electron density itself is typically calculated based on a simple pair potential that maps distance between atoms to the corresponding contribution to the local electron density.

Overall multibody potentials allow a much better reproduction of the elastic properties of metals than pair potentials [105]. However, most conventional multibody potentials are not capable of modelling any effect of directional bonding. To address these effects, modified embedded atom potentials (MEAM) have been proposed that can be parameterised, for instance for silicon [108].

\subsubsection{Integration Time Step}

The time step $\delta t$ used in the integration routine is an important parameter in an MD simulation. A longer time step means that less computer time is required to simulate a given period of real time. On the other hand, errors in the numerical integration increase with the length of the time step. The truncated Taylor series underlying the Verlet algorithm has a local truncation error in atom position of order $O\left(\delta t^{4}\right)$ at each time step. During the course of a simulation the local truncation errors aggregate, resulting in a global truncation error of order $O\left(\delta t^{2}\right)$ over a series of time steps [109]. This shows how these errors decrease as $\delta t$ tends to zero, but not how well the numerical integrator tracks the correct dynamics during a simulation. If, for a given length of time step $\delta t$, the global truncation error grows without bound during the course of a simulation, the results of the numerical integration will diverge from the correct dynamics, e.g. unbounded growth in the energy, invalidating the simulation. In order to assess the effect of the length of the time step on the global truncation error, it is common practice to use a harmonic oscillator as a test case for the numerical integration method, as discussed in [110], [111] and [112]. For the case of the Verlet integrator applied to a damped harmonic oscillator with angular frequency $\omega$ and damping parameter $\gamma$, Zhang and Schlick [111] show that the time step must satisfy the following limit

$$
\delta t \leq \frac{2 \omega-\gamma}{\omega^{2}}
$$


in order to prevent unbounded growth in the global truncation error. Thus the time step $\delta t$ is limited by the frequency of oscillation. We estimate the frequency of oscillation for the Lennard-Jones system using the harmonic oscillator as a model. In the following calculations Lennard-Jones reduced units are used. For most simulations in this work the reduced temperature is $T=0.35$, although in some cases temperatures up to 0.50 are used. Using the equipartition theorem with $k_{B}=1$, the temperature $T=0.50$ corresponds to an average kinetic energy of 0.75 . Consider a harmonic oscillator at its equilibrium position at the bottom of its potential well where its energy of 0.75 is all kinetic energy. As it oscillates away from equilibrium the kinetic energy is converted to potential energy, until at its maximum displacement the kinetic energy is zero and the potential energy is now 0.75. In the case of the Lennard-Jones potential with energy -1 at the bottom of the potential well, this maximum displacement corresponds to a potential energy of -0.25 . The Lennard-Jones potential rises to -0.25 when atoms either move a little closer together than the equilibrium spacing of $2^{\frac{1}{6}}$, or when they move significantly further apart than the equilibrium spacing. We consider the case of closer approach, as there the force is the strongest leading to a higher frequency of oscillation and consequently lower bound on the time step. At a separation 1.012 i.e. approximately $90 \%$ of the equilibrium spacing of $2^{\frac{1}{6}}$, the potential energy is -0.25 . The effective spring constant i.e. the slope of the force, at separation 1.012 is $K_{L J}=376.5$ and the atom mass is 1 , giving a corresponding frequency of oscillation of $\omega=\sqrt{\frac{376.5}{1}}=19.4$ in the case of a harmonic oscillator. Therefore with $\gamma=1$ the upper bound on $\delta t$ from Eq. (3.9) is 0.10. As this upper bound has been estimated using a harmonic oscillator model and without any allowance for fluctuations in the average energy it was deemed prudent to set the time step significantly lower than the upper bound. Consequently in this work the reduced time step was set at $\delta t=0.01$. We note that this size of time step has been shown to be effective in other work using the Lennard-Jones potential and Verlet integrator as discussed in Heerman [113]. Also, Allen and Tildesley [64] demonstrate that with a time step of $\delta t=0.01$ the Verlet integrator only produces small short term fluctuations in energy, i.e. root mean square energy fluctuations of approxi- 
mately 0.01. Furthermore, with the short term energy fluctuations well controlled there will not be any long term drift in energy, because the Verlet integrator is a symplectic integrator. The advantage of a symplectic integrator is that it conserves phase volume throughout the time evolution of the system. As a result, the Verlet integrator rigorously conserves a pseudo-Hamiltonian of the sytem. The difference between the conserved pseudo-Hamiltonian and the true Hamiltonian (energy) is of the order of $\delta t^{2}$, thus avoiding long term energy drift [114].

\subsubsection{Computational Cost}

From the equations of motion Eq. (3.1) it follows that in a direct force calculation over all pairs of atoms, the calculation cost scales as $O\left(N^{2}\right)$ for $N$ atoms. Therefore, the force calculation is the most computationally expensive part of an MD simulation and it is very important in practice to make this calculation as efficient as possible. One way to reduce the computational cost is to express the force in analytic form, rather than carrying out a differentiation of the potential at each time step. A further very important step to reduce the cost is the use of a cut-off distance where appropriate. Atoms which are separated by more than this cut-off distance do not have any interaction. Force calculations for any given atom are then limited to only those atoms within the cut-off distance of the given atom. Use of a cut-off distance therefore reduces the number of force pair calculations per time step. Employing a cut-off distance is only suitable when the potential rapidly reduces to a negligible size within a short distance from an atom. Otherwise the forces on a given atom due to atoms beyond the cut-off distance may be significant, in which case disregarding such forces could invalidate the simulation. The Lennard-Jones potential used in this work is short ranged and so a cut-off distance of the order of $2.5 \sigma$ is commonly employed [64]. In this work the cut-off was set to $3.0 \sigma$.

When a cut-off is used the force calculation at each time step for each atom $\mathbf{i}$ involves checking the distance between atom $\mathbf{i}$ and every other atom and then carrying out a force calculation if the distance is within the cut-off. Since only a 
few atoms are within the cut-off distance of a given atom, most of these checks do not contribute to the force on the given atom. Therefore the computational cost of the force calculation can be further reduced by avoiding these unneeded checks. This can be achieved by maintaining, for each atom, a neighbour list of the other atoms it interacts with, and limiting the force calculations to the lists. These lists need to be updated at intervals to take into account any movement of neighbouring atoms into or out of the cut-off distance. This use of neighbour lists was suggested by Verlet [115]. Through limiting the atom pair distance calculations and force calculations to each of the $N$ atoms and their respective neighbour lists the calculation cost scales as $O(N)$. The MD simulation code used in this work employs such neighbour lists for computational efficiency.

\subsubsection{Visualisation}

It is very useful to be able to visually inspect the changes in atomic configurations that occur during a simulation run. This visual information facilitates the discovery of interesting or novel atomic rearrangements and can help gain an understanding of how observed rearrangements arise.

To accomplish this visualisation the coordinates of all the atoms in the simulation are written to a file at regular intervals during a simulation run. These coordinate files are then read by a visualisation program such as gopenmol [116] which can display each atom as a sphere centered on its coordinates. The configuration of visualised atoms can be translated, rotated and enlarged within the visualisation program as desired to aid inspection. Individual atoms in the configuration can be color coded based on factors such as the number of nearest neighbour atoms. These visualised atom configurations can be saved as image files. The atom configuration figures in this thesis were produced using this method. 


\subsubsection{Combining Methodologies}

A key element in this work is the implemention of a hybrid methodology which dynamically combines both Molecular Dynamics and Kinetic Monte Carlo within a single simulation. The details of this hybrid method are covered in Chapter 5 Hybrid MD KMC Model. This section discusses how different methodologies may be combined.

When two methods are being employed in concert, the most useful approach is to apply the separate methods in spatially separate domains within the simulation. Where domains hosting different methods meet, arrangements must be made to combine the two simulation methods. A common appproach is to establish a 'handshaking region' between each pair of different domains. A transition is made between the simulation methods across each handshaking region. Such a transition is most readily achieved when the two methodolgies are very similar in nature, for example between two Molecular Dynamics methods with different potential functions.

Buehler $[117,118]$ employed handshaking regions to transition between the ReaxFF force field and the Tersoff potential to implement Multiparadigm Molecular Dynamics simulations of crack propagation in Silicon. Across the handshaking region the force and energy contribution from the two formulations were weighted, giving rise to a smooth transition between ReaxFF and Tersoff. The weights were set by linear interpolaton.

The hybrid method in this work makes use of two very different methodolgies. Molecular Dynamics uses an interatomic potential function to determine forces and velocities, while Kinetic Monte Carlo employs transition rates to move atoms between lattice sites. As a consequence it is not feasible to readily implement a transition between the methods across a handshaking region. In this work the two methods are combined as follows. Molecular Dynamics domains treat adjacent Kinetic Monte Carlo domains as if they were Molecular Dynamics domains composed of stationary (frozen) atoms. Kinetic Monte Carlo domains interact with adjacent Molecular Dynamics domains by treating the Molecular Dynamics atoms as if they were located on Kinetic Monte Carlo lattice sites. This 
approach has been effective in combining these two distinct simulation methodologies, and it allows for switching the methodology applied to a given domain during a simulation run as needed.

\subsection{Transition State Theory (TST)}

Transition state theory is a method for calculating the rate of occurrence of rare events.

In many systems of interest, the system spends a large part of the time residing in one of a number of local potential energy minima, with infrequent shifts between these minima, e.g. an adatom diffusing across a surface. Calculating the rates of such shifts is of considerable importance, and Transition State Theory is one method of doing so.

Transition State Theory was originally developed by Eyring [119], [120] to more accurately describe chemical reactions. In this theory, during a chemical reaction the initial reactants which are in a stable state with low potential energy interact to form an activated complex (a transition state). This activated complex has high potential energy and is unstable. The activated complex rapidly decays to the stabler (lower potential energy) products (and possibly some reactants). The theory was extended to solids by Wert and Zener [121] and Vineyard [122].

Again, as with other subjects in this work, only the main aspects and features of Transition State Theory (TST) relevant to this work will be discussed here. An extensive review of transition state theory is to be found in [123], and TST is described in more detail in [124],[125], and [126].

\subsubsection{TST Assumptions}

The basic assumptions of TST are:

i) there exists a (hyper)surface in the phase space which divides the space into a reactant region and a product region (this dividing surface contains the transition state); 
ii) trajectories that pass through this dividing surface in the direction of the products which originated at reactants, will not re-cross the surface in the opposite direction (the no-recrossing assumption);

iii) the reactants and the transition state are equilibrated in a canonical (fixedtemperature) ensemble; and

iv) the reaction is electronically adiabatic (i.e. according to the Born-Oppenheimer approximation the electronic state does not change during the transition).

Eyring demonstrated that when these assumptions hold, the rate $\Gamma_{r p}$ for the transition from reactants to products $M_{r} \Rightarrow M_{p}$, is given by

$$
\Gamma_{r p}=\frac{k_{B} T}{h} \frac{Z_{q}}{Z_{r}}
$$

where $Z_{q}$ and $Z_{r}$ are the canonical partition functions of the transition state and of the initial state (reactants) respectively, and h is Planck's constant.

Using the canonical expression for the Helmholtz free energy $F=-k_{B} T \ln Z$, the rate can be written in Arrhenius law form

$$
\Gamma_{r p}=\frac{k_{B} T}{h} \exp \left(-\frac{F\left(M_{q}\right)-F\left(M_{r}\right)}{k_{B} T}\right) .
$$

Now the Helmholtz free energy can be written as $F=U-T S$. The internal energy $U$ of a crystal lattice consists of a static part $E$ and a vibrational part $U_{v i b}$, and the entropy is vibrational entropy $S_{v i b}$. The rate of a process can therefore be expressed as:

$$
\begin{gathered}
\Gamma_{r p}=\Gamma_{r p}^{o} \exp \left(\frac{\triangle E}{-k_{B} T}\right) \\
\text { where } \Gamma_{r p}^{o}=\frac{k_{B} T}{h} \exp \left(-\frac{\triangle U_{v i b}}{k_{B} T}+\frac{\triangle S_{v i b}}{k_{B}}\right) .
\end{gathered}
$$

The static energy difference $\triangle E$ can represent a diffusion or desorption energy barrier. The prefactor $\Gamma_{r p}^{o}$ is usually interpreted as an attempt-to-escape frequency from the reactant configuration. 


\subsubsection{Harmonic TST}

In the practical application of Eq. (3.10) to surface diffusion, one usually considers the surface as a heat bath of harmonic oscillators. At $M_{r}$, the system is described by $N$ normal modes with angular frequencies $\omega_{n}^{r}$. At the saddle point $M_{q}$, however, the mode corresponding to traversing the reaction coordinate which connects the saddle point with the reactants and products has an imaginary frequency and has to be excluded. Thus at $M_{q}$ there are $N-1$ normal modes with frequencies $\omega_{n}^{q}$. In the classical limit $k_{B} T \gg \hbar \omega_{n}$, the analytical expressions for $U_{v i b}$ and $S_{v i b}$ can be simplified. As a result the attempt frequency $\Gamma_{r p}^{o}$ can be expressed by the frequency-product formula developed by Vineyard [122]:

$$
\Gamma_{r p}^{o}=\frac{1}{2 \pi} \frac{\prod_{n=1}^{N} \omega_{n}^{r}}{\prod_{n=1}^{N-1} \omega_{n}^{q}} .
$$

In practice, in most of the works that deal with the theoretical computation of process rates, the prefactors are chosen as site-independent constants. This choice is adopted in part because it is computationally expensive to evaluate the attempt frequencies from first principles, but also because the relevant phonon modes are not expected to vary much from site to site, while the exponential term $\exp \left(-\frac{\Delta E}{k_{B} T}\right)$ can easily vary by several orders of magnitude. Moreover, even if the attempt frequencies were known accurately, the uncertainty in the calculated energy barrier of approximately \pm 0.1 would result in an exponentially large uncertainty $\exp \left(\frac{ \pm 0.1}{k_{B} T}\right)$ in the jump (transition) rate. Therefore, for qualitative analysis it is usually only necessary to obtain an order of magnitude estimate for $\Gamma^{o}$. This can be obtained by using the Debye temperature $\Theta_{D}$ of the substrate material as $\Gamma^{o} \approx k_{B} \Theta_{D} / h$, or by using Vvedensky's harmonic approximation [127]: $\Gamma^{o} \approx 2 k_{B} T / h$. The attempt frequencies are more often equated to the maximum phonon frequency, whose typical values in the physical systems of interest are approximately $10^{13} \mathrm{sec}^{-1}$. 


\subsection{The Nudged Elastic Band method}

\subsubsection{Finding the Minimum Energy Path}

In the previous section we discussed the relationship between the diffusion energy barrier $\triangle E$ and the rate of transition between reactant and product states. This energy $\triangle E$ is the minimum energy required for a transition to occur. To calculate $\triangle E$ it is necessary to know the potential energy at the transition state and at the minima. Transition states occur at saddle points between the lower energy states occupied by the reactants and products.

In this section we consider the problem of finding the Minimum Energy Path (MEP), connecting a pair of local potential energy minima via a saddle point between them. The maximum potential energy along the MEP is the potential energy at the saddle point (transition state). Therefore, knowledge of the MEP enables calculation of the diffusion energy barrier $\triangle E$. The MEP gives the the reaction path followed by the system in its transition. Also, a reaction coordinate can be defined as the distance along the MEP.

The Nudged Elastic Band method (NEB) developed by Hannes Jónsson and co-workers $[128,129,130]$ is an efficient method for finding the MEP. The NEB method belongs to the class of chain-of-states methods in which a chain of images (or replicas) of the $3 N$-dimensional system is generated between the end point configurations. All the intermediate images are optimised simultaneously in a concerted way, in order to move the path they form towards the MEP, while the end points remain fixed. Typically the initial images are located along the line joining the initial and final points. The optimisation is carried out by simultaneous annealing of all the images. This relaxes each image to its lowest energy configuration. After the optimisation the images lie near the MEP. The distribution of the images, which gives a discrete representation of the path, can be controlled and can be set to be higher (images closer together) in the saddle point region as compared with the end regions.

The NEB is an improvement of the Elastic Band method, in which the images are connected with springs of zero natural length, so the force acting on image $i$ 
is:

$$
\mathbf{F}_{\mathbf{i}}=-\nabla E\left(\mathbf{R}_{\mathbf{i}}\right)+\mathbf{F}_{\mathbf{i}}^{\mathbf{s}}
$$

where $\mathbf{R}_{\mathbf{i}}$ is a $3 N$-dimensional coordinate, and $\mathbf{F}_{\mathbf{i}}^{\mathbf{s}}$ is the spring force:

$$
\mathbf{F}_{\mathbf{i}}^{\mathbf{s}}=k_{i+1}\left(\mathbf{R}_{\mathbf{i}+\mathbf{1}}-\mathbf{R}_{\mathbf{i}}\right)-k_{i}\left(\mathbf{R}_{\mathbf{i}}-\mathbf{R}_{\mathbf{i}-\mathbf{1}} .\right)
$$

Two problems that can arise from this approach are known as "corner cutting" and "sliding down". The problem of "corner cutting" results from the component of the spring force which is perpendicular to the path, and tends to pull images off the MEP. The problem of "sliding down" results from the component of the true force $-\nabla E\left(\mathbf{R}_{\mathbf{i}}\right)$ in the direction of the path. The cure for these undesired effects proposed by Hannes Jónsson et al. is to include only the perpendicular component of the true force $-\nabla E\left(\mathbf{R}_{\mathbf{i}}\right) \perp$, and the parallel component of the spring force $\mathbf{F}_{\mathbf{i}}^{\mathbf{s}} \|$. The total force acting on image $\mathbf{i}$ is thus reduced to:

$$
\mathbf{F}_{\mathbf{i}}=-\nabla E\left(\mathbf{R}_{\mathbf{i}}\right) \perp+\mathbf{F}_{\mathbf{i}}^{\mathbf{s}} \|
$$

where

$$
\begin{gathered}
-\nabla E\left(\mathbf{R}_{\mathbf{i}}\right) \perp=\nabla E\left(\mathbf{R}_{\mathbf{i}}\right) \cdot \hat{\tau}_{i}-\nabla E\left(\mathbf{R}_{\mathbf{i}}\right) \\
\mathbf{F}_{\mathbf{i}}^{\mathbf{s}} \|=\left[k_{i+1}\left|\mathbf{R}_{\mathbf{i}+\mathbf{1}}-\mathbf{R}_{\mathbf{i}}\right|-k_{i}\left|\mathbf{R}_{\mathbf{i}}-\mathbf{R}_{\mathbf{i}-\mathbf{1}}\right|\right] \cdot \hat{\tau}_{i}
\end{gathered}
$$

with $\hat{\tau}_{i}$ being the tangent to the path at point $\mathbf{R}_{\mathbf{i}}$. These force projections, referred to as "nudging", decouple the action of the true force on the path from that of the spring force. The force in Eq. (3.13) pulls the elastic band onto the MEP, while the force in Eq. (3.14) controls the distribution of the images along the MEP. The spring force does not interfere with the convergence of the elastic band to the MEP. The strength of the spring forces can be varied by several orders of magnitude without affecting the equilibrium position of the band.

\subsubsection{Enhancements}

\subsubsection{Tangent Estimate}

When the energy of the system changes rapidly along the path, but the restoring force on the images perpendicular to the path is weak, as can be the case when 
covalent bonds are broken and formed, kinks can form on the elastic band.

If we compute the local tangent of the path as

$$
\hat{\tau}_{i}=\frac{\mathbf{R}_{\mathbf{i}+\mathbf{1}}-\mathbf{R}_{\mathbf{i}-\mathbf{1}}}{\mathbf{R}_{\mathbf{i}}-\mathbf{R}_{\mathbf{i}-\mathbf{1}}}
$$

then the perpendicular component of the true force Eq. (3.13) acting on the two images that are adjacent to the image $\mathbf{i}$ will be affected by the presence of the kink. As the minimisation algorithm is applied, the wrong movement of the two adjacent images will cause the kink to continue oscillating back and forth, preventing the band from converging to the MEP.

The tangent to the path at the image $\mathrm{i}$ is then chosen according to the prescription [131]

$$
\hat{\tau}_{i}=\frac{\left(\mathbf{R}_{\mathbf{i}+\mathbf{1}}-\mathbf{R}_{\mathbf{i}-\mathbf{1}}\right)}{\left(\mathbf{R}_{\mathbf{i}}-\mathbf{R}_{\mathbf{i}-\mathbf{1}}\right)} \quad \text { if } E_{i+1}>E_{i}>E_{i-1}
$$

which makes the tangent point from image $\mathbf{i}$ to the adjacent image that has the higher energy. The tangent is then normalized. This choice ensures that the highest energy image between those adjacent to a kink is not affected in its motion by the presence of the kink. The choice for the local tangent to be determined by the higher energy neighbouring image is motivated by the fact that the MEP can be found by following the force lines down the potential from the saddle point, but never up from a minimum.

\subsubsection{Climbing Image}

When the elastic band converges to the MEP, typically none of the images lands at the saddle point, and the saddle point energy needs to be estimated by interpolation. "Climbing image" [132] is a slight modification to the NEB algorithm that makes one of the images climb up along the elastic band and converge rigorously on the highest saddle point. After a few NEB iterations, the image $\mathbf{i}_{\text {max }}$ with the highest energy is identified. The force acting on this image is not given by Eq. 3.12 , but rather by

$$
\mathbf{F}_{\mathbf{i}_{\text {max }}}=-\nabla E\left(\mathbf{R}_{\mathbf{i}_{\text {max }}}\right)-2 \nabla E\left(\mathbf{R}_{\mathbf{i}_{\text {max }}}\right) \|=-\nabla E\left(\mathbf{R}_{\mathbf{i}_{\text {max }}}\right)-2 \nabla E\left(\mathbf{R}_{\mathbf{i}_{\text {max }}}\right) \cdot \hat{\tau}_{i} .
$$


This is the true force due to the potential with the component along the elastic band inverted. The spring force does not act on the maximum energy image. Therefore, the spacing of the images will be different on each side of the climbing image. As it moves up to the saddle point, images on one side will get compressed, and on the other side spread out.

\subsubsection{Variable Spring Constants}

Since the saddle point is the most relevant point along the MEP, it is important to get a good estimate of the tangent to the path near the saddle point, especially when climbing the image. This can be accomplished by using stronger spring constants near the saddle point. As we have already explained, the choice of the spring constant does not affect the saddle point position, thus one is free to choose their values in different ways. The choice suggested in [132] is to choose spring constants that depend linearly on the energy of the image. In particular one has to set a range for spring constant variability $\delta k=k_{\max }-k_{\min }$, then the spring acting between two adjacent images is

$$
k_{\mathbf{i}}=k_{\max }-\delta k\left(\frac{E_{\max }-E_{\mathbf{i}}}{E_{\max }-E_{\text {ref }}}\right) \quad \text { if } E_{\mathbf{i}}>E_{\text {ref }} .
$$

Here $E_{i}$ is the higher energy of the two images connected by spring $\mathbf{i} ; E_{\max }$ is the higher energy value along the band; $E_{\text {ref }}$ is a reference value that can be set at the highest energy between the two endpoints of the MEP [132]. By choosing $E_{\mathbf{i}}$ to be the higher energy of the two images connected by the spring, the two images adjacent to the climbing image will tend to be almost symmetrically arranged around the saddle point. 


\subsection{Simulation Time Scale}

The physical phenomena that determine the surface evolution during crystal growth take place with mesoscopic or macroscopic length scales, and over time scales of seconds or even minutes. However the physics and chemistry that govern the behavior of an atom on a surface operate on the length scale of the chemical bond, i.e. $10^{-10}$ meters, and with time scales of atomic vibrations, i.e. approximately $10^{-13}$ seconds. Atomistic simulations of crystal growth therefore require bridging the gap between a microscopic and a mesoscopic or macroscopic description. Large time scales are involved because thermally activated, rare events predominate. The time period between two diffusion processes, e.g. adatom hops, depends on the temperature and crystal surface facet. This time period can extend to nanoseconds. This rare events problem precludes the use of a direct Molecular Dynamics approach.

\subsubsection{Accelerated Molecular Dynamics}

When the dynamics of a system are determined by rare events, the system spends a large part of the time residing in one of a number of local potential energy minima, with infrequent transitions between these minima. For such systems, Voter has developed three accelerated Molecular Dynamics methods to extend the time scale that can be simulated. These methods are designed to increase the likelihood of the rare events occurring. Therefore more rare events and effectively longer simulation times, occur in a given amount of computer time, when compared to standard Molecular Dynamics.

The first method is called Hyperdynamics [133]. In Hyperdynamics, the potential energy surface of the system is modified by adding to it a non-negative bias potential. A constant temperature Molecular Dynamics trajectory is then propagated on this modifed potential energy surface. The method requires that the system obeys TST. Also the bias potential must be zero at all the dividing surfaces, and the system must still obey TST for dynamics on the modified surface. A trajectory on such a modified surface, while relatively meaningless on the atomic 
vibrational time scale, evolves correctly from state to state at an accelerated pace. The evolution is correct in the sense that the probability of observing any particular sequence of states is the same for a simulation on the modified potential as for the original potential. In Hyperdynamics, the time advance at each step equals the regular Molecular Dynamics time step multiplied by an instantaneous boost factor, the inverse Boltzmann factor for the bias potential at that point. A major difficulty with this method is the construction of a suitable bias potential for a given system.

The second of Voter's methods is Parallel Replica Dynamics [134]. This method is the simplest and most accurate of the three accelerated Molecular Dynamics techniques, with the only assumption being that of infrequent events obeying first order kinetics (exponential decay). That is, for any time greater than decorrelation time $\tau_{\text {corr }}$ after entering a state, the probability distribution function for the time of the next escape is given by

$$
p(t)=k \exp (-k t)
$$

where $k$ is the rate constant for escape. Starting with an $\mathrm{N}$ atom system in a basin around a local potential energy minima, the entire system is replicated on each of $\mathbf{M}$ available processors. After a short dephasing stage $\left(\tau_{\text {dephase }}>\tau_{\text {corr }}\right)$, during which momenta are periodically randomized to eliminate correlations between replicas, each processor carries out an independent constant temperature Molecular Dynamics trajectory for the entire $\mathrm{N}$ atom system, thus exploring phase space within the particular basin $\mathrm{M}$ times faster than a single trajectory would. Whenever a transition out of the basin is detected on any processor, all processors are alerted to stop. The simulation clock is advanced by the accumulated trajectory time summed over all replicas, i.e. the total time spent exploring phase space within the basin until an escape pathway is found.

This method also correctly accounts for correlated dynamical events, i.e. there is no requirement that the system obeys TST. This is accomplished by allowing the trajectory that made the transition to continue on its processor for a further amount of time $\left(\Delta \tau>\tau_{\text {corr }}\right)$, during which recrossings or follow on events may 
occur. The simulation clock is then advanced by $\Delta \tau$, the final state is replicated on all processors, and the whole process is restarted. This overall procedure then gives exact state to state dynamical evolution because the escape times obey the correct probability distribution; nothing about the procedure corrupts the relative probabilities of the possible escape paths, and the correlated dynamical events are properly accounted for. A key requirement for this method is an effective transition detection scheme.

The efficiency of the method is limited by both the dephasing stage, which does not advance the system clock, and the correlated event stage, during which only one processor accumulates time.

The third method is Temperature Accelerated Dynamics (TAD) [135]. The idea of this method is to speed up the transitions by increasing the temperature, while filtering out the transitions that should not have occurred at the original temperature. The TAD method is more approximate than the previous two methods in that it relies on the harmonic TST approximation. The TAD method often gives substantially more boost than Hyperdynamics or Parallel Replica dynamics. The trajectory in TAD is allowed to wander on its own to find each escape path, so that no prior information is required about the nature of the reaction mechanisms. In each basin, the system is evolved at a high temperature $T_{\text {high }}$ (while the temperature of interest is some lower temperature $T_{l o w}$ ). Whenever a transition out of the basin is detected, the saddle point for the transition is found, e.g. using the Nudged Elastic Band method. The trajectory is then reflected back into the basin and continued. This procedure generates a list of escape paths and attempted escape times for the high temperature system. Assuming that TST holds and that the system is ergodic, the probability distribution for the first escape time for each mechanism is an exponential. Because harmonic TST gives an Arrhenius dependence of the rate on temperature, depending on the saddle point energy barrier, the escape time observed at $T_{\text {high }}$ can be extrapolated to obtain a corresponding escape time at $T_{\text {low }}$ that is drawn correctly from the exponential distribution at $T_{\text {low }}$. The event with the shortest time at low temperature is the correct transition for escape from this basin. For a chosen level of confidence that the shortest time 
has been found, a stop time is determined. Once this stop time is reached, the system clock is advanced by the shortest time at $T_{\text {low }}$, the corresponding transition is accepted, and the TAD procedure is started again in the new basin.

\subsubsection{Autonomous Basin Climbing Method}

This method for speeding up molecular dynamics was developed by Kushima, Yip and others [136]. This is a potential energy surface sampling method developed to simulate slow dynamical processes without explicitly following all the atomic rearrangements all the time. The method requires the input of an interatomic interaction potential for the constituent particles in the system, and retains full information on the particles along the trajectory of the sampling. The system is evolved by a series of elementary steps of alternating activation and relaxation in the space of its potential energy, which allows the system to climb out of any potential well. On the potential energy surface where there are many local minima and saddle points, having the ability to climb out of any potential well means that one can then sample the topography of the potential energy surface by generating an explicit transition state pathway trajectory. The system evolution, on an energy scale, is therefore described by this trajectory.

A series of the two elementary steps of activation and relaxation move the system up a particular potential well. Firstly a $3 N$ dimensional Gaussian penalty function ( $N$ is the number of atoms in the system) is added to an initial energy minimised configuration. This has the effect that upon the following relaxation step (minimisation of the combined energy of the penalty and the original potential energy), the system is pushed away from the initially energy-minimised configuration into a higher energy state. With sufficient energy penalties accumulated after a series of such steps, the system will cross an energy saddle point during a relaxation step and enter into an adjacent potential well. Starting at the bottom of this well the activation-relaxation series is repeated until the system is able to escape from the second well. Since the energy penalty functions imposed in the previous activation steps are not removed during the entire sampling, the 
system is always discouraged from returning to any previously visited potential minima.

In this method one is not sampling a fixed potential energy surface; rather the potential energy surface evolves along with the sampling such that the system is always encouraged to sample new regions of the potential energy surface. In contrast to saddle point sampling techniques such as the NEB method, this method does not require prior knowledge of the final state. This is particularly useful for systems where the structure is too complex to generate such an input. This method has be applied to study activated kinetics of solid-state deformations under stress [137].

\subsection{Kinetic Monte Carlo}

\subsubsection{Uses and Features of the KMC Methodology}

Surface evolution during crystal growth is dominated by rare events such as adatom diffusion. During the long time period between rare adatom diffusion events, the adatom undergoes a large number of vibrations around its local minima in the potential energy surface. These vibrations are irrelevant from the point of view of surface evolution. The surface only alters when atoms or adatoms move from one site to another. Hence there is no need for a detailed description of the adatom motion during the time the adatom resides in a local minimum.

This opens the way for approaches such as Kinetic Monte Carlo [138], [139], [140]. Bridging of time scales can thus be realised, by using microscopic parameters estimated using NEB as input data for KMC simulations.

The activation energy $E_{a}$ for each identified process can be calculated by the NEB method, as previously discussed. These calculated energy barriers $E_{a}$ are input parameters for KMC simulations, where at each step a particular process is randomly selected with a probability proportional to the process rate. The process rate is defined according to transition state theory: 


$$
\Gamma=\Gamma_{o} \exp \left(-\frac{E_{a}}{k_{B} T}\right)
$$

\subsubsection{Basic Idea of KMC}

The standard Monte Carlo (MC) technique [140], is a methodology for calculating chosen averages in a given equilibrium thermodynamic ensemble. States in the configuration space are generated and used for the calculation of the quantities of interest. These states, although generated sequentially, do not necessarily correspond to the dynamics of the system. Some 30 years ago Monte Carlo simulations started to be applied to the study of kinetic processes [141], [142]. The aim of KMC simulations is to reproduce nonequilibrium dynamics. In KMC the selected configurations correspond to the real configurations the system goes through during its evolution.

MC techniques can be viewed as methods for solving the master equation describing the evolution of the system from a given configuration $C$ :

$$
\frac{\partial P(C, t)}{\partial t}=-\sum_{C^{\prime}} W\left(C \rightarrow C^{\prime}\right) P(C, t)+\sum_{C^{\prime}} W\left(C^{\prime} \rightarrow C\right) P\left(C^{\prime}, t\right)
$$

where $P(C, t)$ is the distribution of configurations at time t, and $W\left(C \rightarrow C^{\prime}\right)$ is a matrix of transition probabilities connecting two states. The transition probability in standard MC simulations does not need to have any relationship to the dynamics of the system. At each MC step a trial configuration is generated, which is then either accepted or rejected. A sequence of configurations forms a Markov chain, which is used for calculating the desired quantities. The condition of detailed balance

$$
W\left(C \rightarrow C^{\prime}\right) P(C, t)=W\left(C^{\prime} \rightarrow C\right) P\left(C^{\prime}, t\right)
$$

which ensures that the ratio of the "forward" and the "backward" probabilities is equal to the ratio of the "forward" and the "backward" transition probabilities, is 
a sufficient condition for the convergence of the Markov chain. Detailed balance holds at steady states and both MC and KMC satisfy detailed balance.

In KMC, configuration changes correspond to real events in the stochastic system. Each of the events can happen with a probability per unit of time (process rate), given by Eq. (3.15). The total rate $R$ for the system to change from configuration $C$ to some other configuration is given by the sum of the rates of the $\mathrm{N}$ processes that are possible at configuration $C$ :

$$
R=\sum_{j}^{N} \Gamma_{j}
$$

where $\Gamma_{j}$ is the rate for process $j$.

\subsubsection{KMC Scheme for Crystal Growth}

When KMC is used to simulate crystal growth, a discrete geometry is usually employed. Atoms can only occupy positions on a discrete lattice, which has the geometry of the crystal. This choice is justified if the material retains its basic crystalline structure during epitaxial growth. In such cases we are only interested in the motion of the atoms forming the crystal, and the growth proceeds by the addition of atoms at unoccupied adsorption positions. Such is the case for example in Molecular Beam Epitaxial growth, where the surface is directly bombarded by atoms.

In $\mathrm{KMC}$, atoms can move from one lattice site to another (diffusion) or appear on or disappear from the surface (adsorption and desorption) by discrete jumps. The motion of individual atoms takes place instantaneously and the motions are independent and Markovian i.e. no memory of previous position. By only considering the jumps, information about atom dynamics between these rare events (jumps) is eliminated. That is, the atom vibrations and the associated time scales are not explicitly modelled. This information is, however, taken into account in the attempt frequency $\Gamma_{o}$. 


\subsubsection{KMC Algorithm}

The basic KMC algorithm was formulated by Bortz et al. [138]. In this work we follow the version developed by Schulze [21, 22]. A simple description of the $\mathrm{KMC}$ algorithm is:

1. Determine all processes $\mathrm{j}$ that possibly could take place - based on the current configuration of the system.

2. Calculate the total rate $R=\sum_{j} \Gamma_{j}$, where the sum runs over the possible processes identified at step 1. Deposition can be one of these processes.

3. Choose two random numbers $\rho_{1}, \rho_{2}$ in the range $(0,1]$.

4. Find the integer number $l$ for which

$$
\sum_{j=1}^{l-1} \Gamma_{j} \leq \rho_{1} R<\sum_{j=1}^{l} \Gamma_{j} .
$$

5. Carry out process $l$, e.g. move an atom.

6. Update the simulation time $t=t+\Delta t$ with $\triangle t=-\ln \left(\rho_{2}\right) / R$.

7. Go back to step 1.

Two important features are the following: firstly, the algorithm for process selection (step 4), ensures that the selected process is chosen randomly with a probability given by the ratio $\Gamma_{j} / R$ of its rate and the total rate for the current configuration of the system. Secondly, in KMC simulations, all physical processes are separated so that at any point in time only one event takes place, and sets of events are generated by Poisson processes. This condition makes the KMC time a physical quantity [143].

The time interval $\triangle t$ between two successive events is a random variable with the distribution $P(\triangle t)=R e^{-\triangle t R}$, and average value of $\langle\Delta t\rangle=1 / R$. Note that the total rate $R$ depends on the system configuration $C$. Step 6 in the algorithm states that the system remains in configuration $C$ for a time interval that is proportional to the inverse of the total rate $R$ of configuration $C$. Thus the larger the total rate for a given process to occur, the shorter the time spent by the system in that configuration. The total rate $R$ also depends on the system temperature. It increases exponentially with the temperature, so that $\Delta t$ as defined in step 6 
becomes very small at high temperatures. This reflects the fact that the events are activated more frequently at higher temperatures. Simulating the system evolution at high temperatures requires a larger number of $\mathrm{KMC}$ steps to simulate a given amount of time.

\subsubsection{Events and Energy Barriers}

A very important aspect of the KMC algorithm used here is that it requires a predetermined list of events and their corresponding energy barriers in order to implement the algorithm. It can be very difficult to determine all the relevant events for some situations, particularly if concerted multiple atom rearrangements are involved. Omission of significant events can distort the results of KMC simulations, as discussed by Voter [65]. Once the list of events has been determined, the relevant energy barriers can be computed using the Nudged Elastic Band method from section 3.4.

In this work, KMC is applied to adatoms diffusing on a (111) facet. A commonly applied approach for determining events and the corresponding energy barriers in such a situation is by way of "bond counting" [140]. In this approach the events are distinguished by the difference between the number of in-plane nearest neighbours of the lattice site an atom is moving from and of the site it is moving to. The energy barrier is composed of an energy term for an isolated adatom plus the energy for the net number of bonds that are broken (one bond per in-plane nearest neighbour) as a result of moving from one site to a neighbouring site.

For the Lennard-Jones potential used in this work, the energy required for an isolated adatom sited in a three-fold hollow site on a (111) facet, to hop to a nearest neighbour three-fold hollow site, was determined using the Nudged Elastic Band method. Each three-fold hollow site on the (111) facet has three adjacent three-fold hollow sites at a distance of $\frac{1}{\sqrt{3}} \times 2^{\frac{1}{6}} \sigma$, and six nearest neighbour threefold hollow sites at $2^{\frac{1}{6}} \sigma$ separation. When a three-fold hollow site is occupied by an adatom, none of its three adjacent sites can also be occupied without causing extremely strong repulsive forces between the occupying adatoms, i.e. an adatom 
"collision". The direct path for an adatom between nearest neighbour sites involves climbing over a surface atom. The NEB calculations show that the MEP between nearest neighbour sites is an indirect "dogleg" path, moving around the intervening surface atom and passing through a adjacent three-fold hollow site, as shown in Figure 3.1.

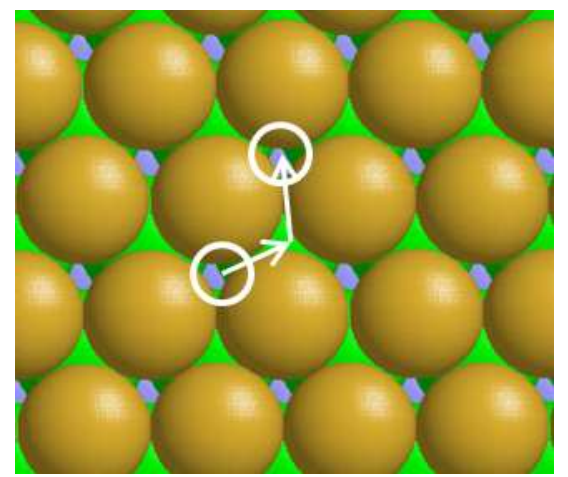

Figure 3.1: On this three layer (111) facet the bottom A layer atoms are blue, second B layer atoms are green and top C layer atoms are gold. The two white circles indicate a pair of nearest neighbour three-fold hollow sites (directly above A layer blue atoms). The white arrows show the "dogleg" minimum energy path for an adatom hopping between these two nearest neighbour sites via an adjacent three-fold hollow site (directly above a B layer green atom).

Figure 3.2 below shows the energy along the minimum energy path for an adatom hopping to a nearest neighbour three-fold hollow site. It is composed of two short hops, the first to the adjacent site and second from the adjacent site to the destination. In Figure 3.2 the dots indicate the positions of the NEB images. The smooth curve fitted through the images shows the MEP. The energy along the MEP is measured relative to the energy of the initial site, i.e. the first image at the origin of the graph. The third image at distance $0.32 \sigma$ along the MEP is the climbing image. It has climbed to the peak of the MEP between the adjacent threefold hollow sites, giving a $0.31 \epsilon$ energy barrier for the first hop. The second peak in the MEP is for the second hop and it has the same energy barrier. Therefore the KMC energy barrier for a nearest neighbour hop is taken to be $0.31 \epsilon$. Note 
that an adatom can make the short hop to an adjacent three-fold hollow just as easily, provided no adatom "collision" would ensue. In Chapter 4 we discuss how making a short hop changes an adatom's stacking, i.e. its relationship to the underlying layers of atoms. Changes in atomic stacking are very important in this work, consequently short hops are explicitly modelled in the KMC used here.

This $0.31 \epsilon$ energy barrier and the energy per bond of $1 \epsilon$ (the depth of the Lennard-Jones potential well) are used with "bond counting" to determine the energy barriers for other events.

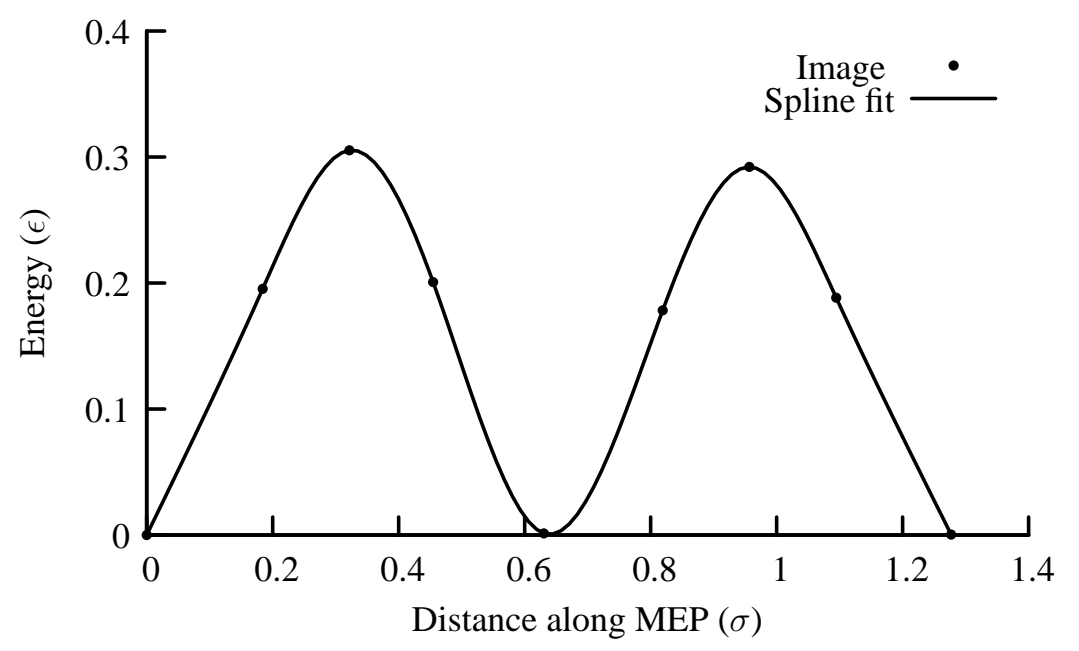

Figure 3.2: Energy along the "dogleg" minimum energy path for adatom nearest neighbour hop on (111) surface.

\subsection{Summary}

The Molecular Dynamics methodology was discussed first. There is a wide range of applications of MD. The dynamics of atoms in an MD simulation follow from (in the simplest case) Newton's equation of motion. The equation of motion is numerically integrated to derive the positions and velocities of the atoms at each time step in the simulation. Suitable boundary conditions are required when bulk 
material is modelled. When the temperature of the simulation is to be controlled, the equation of motion and the integration process need to be modified. The force between pairs of atoms is derived from a potential energy function. In this work the Lennard-Jones potential is used. This is a simple potential widely used as a general first approximation in MD.

The other simulation methodology used here is Kinetic Monte Carlo. Prior to discussing KMC we covered Transition State Theory and the Nudged Elastic Band method. Transition State Theory is a method for calculating the rate of occurrence of rare events. Harmonic Transition State Theory relates the rate of transition to the energy barrier separating distinct atomic configurations. The Nudged Elastic Band method is used to determine these energy barriers. The energy barriers are key parameters in determining the rates for atomic rearrangements and hence atomic configuration changes in KMC simulations. The KMC methodology and algorithm were discussed, as applied to the modelling of crystal growth. 
Chapter 4

Molecular Dynamics Modelling of

Stacking Fault Grain Boundaries 


\subsection{Molecular Dynamics Modelling}

In this chapter we discuss our application of the Molecular Dynamics methodology to simulate the behaviour of stacking fault grain boundaries in an epitaxial layer. This is being done to establish a benchmark for the hybrid MD KMC methodology. The hybrid methodology which is discussed in chapter 5, was structured to be consistent with the MD simulations in this chapter. For the MD simulations discussed here, a configuration of atoms containing stacking fault grain boundaries was constructed. The movements of and changes in these grain boundaries were then simulated using Molecular Dynamics. Many simulations were carried out and inferences were drawn from the aggregate statistics of the results of these simulations.

We discuss the changes in the shape of such grain boundaries with time. The migration of positions of the grain boundaries with time is analysed. The results of the simulations are compared with relevant experimental work, and the key atomic mechanisms for these changes are determined.

\subsection{Atomic Layer Stacking on the (111) Facet}

The Face Centered Cubic (FCC) crystal structure is composed of a cubic lattice with one atom at each vertex of the cube and one atom in the centre of each face of the cube. The lattice spacing is given by the edge length of the cube, and the distance between nearest neighbour atoms is half the length of the face diagonal of the cube. A (111) facet is formed when a block of atoms in the FCC structure is cleaved along a pair of adjacent face diagonals, e.g. for a cube of edge length $a$, the cleavage plane through the three points $(a, 0,0),(0, a, 0)$ and $(0,0, a)$. We now discuss some of the features of the (111) facet, as such facets form the atomic surface used in these simulations.

The (111) facet displays a triangular lattice of hollow sites, each of which has six surrounding hollow sites. Any atoms of the same type deposited on a (111) facet are most strongly bound in these hollow sites, and when this occurs 
epitaxial atomic layers start to form. The threefold nature of the lattice provides three distinct sets of hollow sites for atoms to attach to, as shown in Figure 4.1. These three sets are labelled A-sites, B-sites and C-sites respectively.

For a (111) facet to grow epitaxially while retaining its FCC stacking, adjacent atomic layers must follow the stacking pattern $\mathrm{ABC}$. That is, a layer of atoms all in A-sites is followed by the next layer of atoms all in B-sites, and in the subsequent layer all atoms are in $\mathrm{C}$-sites. This stacking pattern is repeated for all following atomic layers.

Alternatively, after a layer of A-sites has been followed by a layer of B-sites the third layer may be comprised of atoms on A-sites again, rather than atoms on $\mathrm{C}$-sites. This change in stacking pattern from $\mathrm{ABC}$ to $\mathrm{ABA}$ is a stacking fault $[144,145]$ and gives rise to hexagonal close packing (HCP) stacking. Figure 4.1 shows a side view of these different stacking patterns. A top view is shown in Figure 4.2.

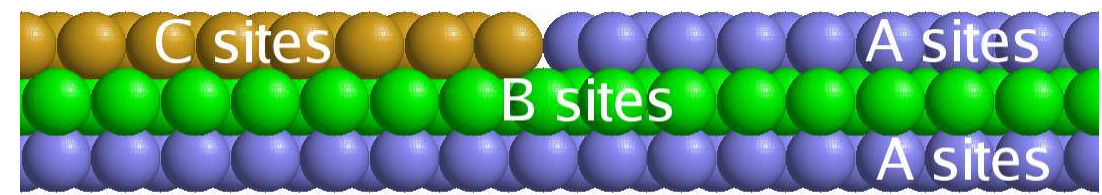

Figure 4.1: Side view of three atomic layers showing the three different stacking sites. Three layers in the ABC (from bottom layer to top layer) stacking pattern (left half of image) give FCC stacking. When the stacking pattern is ABA, HCP stacking results (right half of image). There is a stacking fault grain boundary in the center of the top layer where the two different stackings meet. 


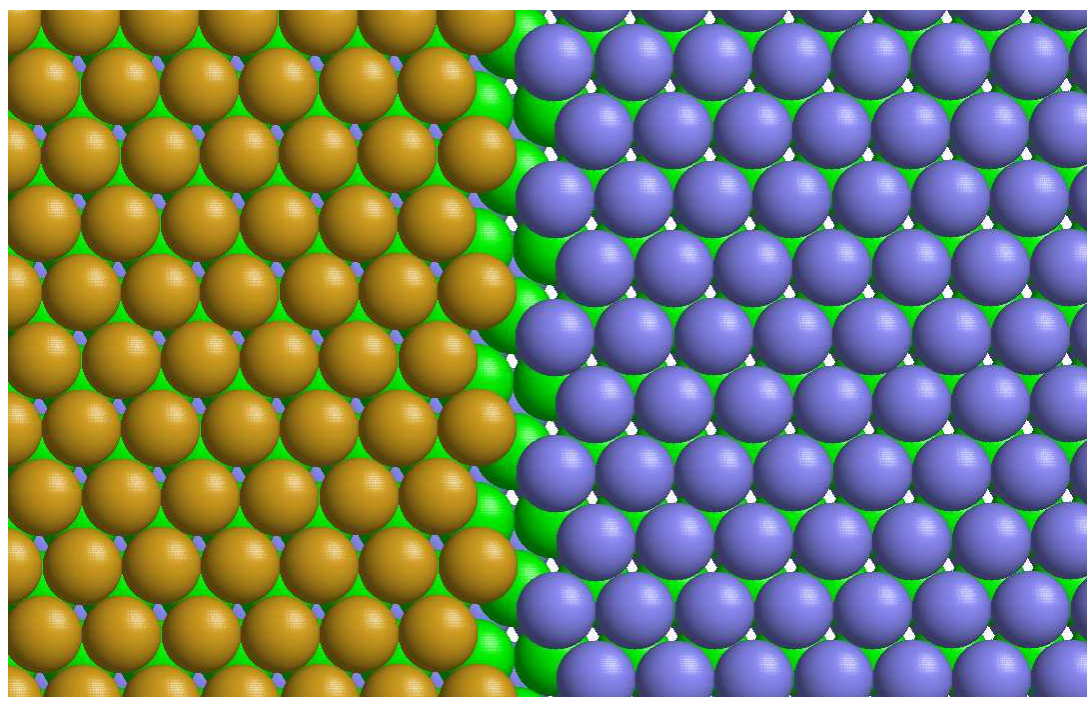

Figure 4.2: Top view of the three atomic layers in Figure 4.1. The left side of the image with gold atoms in the top layer, form the FCC stacking region. On the right side is the HCP stacking region with atoms coloured blue in the top layer. The two different stacking regions are unable to merge, leaving a stacking fault grain boundary (vertical gap in top layer in center of image) between them. On the left side of the image the bottom A layer atoms in blue, second B layer atoms in green and top $\mathrm{C}$ layer atoms in gold can be all be glimpsed. On the right side the top layer blue atoms are located in A sites, placing them directly above the bottom layer A sites. The $\mathrm{C}$ sites are not occupied and so the white background shows through.

Stacking patterns such as ABB are not stable. The interlayer spacing between the atoms in the two adjacent B-site layers is too small, producing strong repulsion between the atoms in these two layers, thereby deranging the epitaxial layers. Hence we need only consider FCC and HCP stacking patterns.

Layers of atoms which only cover part of a (111) facet are known as islands. Islands in the different stackings FCC and HCP may form independently on different parts of a (111) facet. When islands with different stacking grow (due to deposition or movement of adatoms) and meet each other, they are unable to immediately coalesce into a single larger island if they are sufficiently large, because their constituent atoms reside on different sets of sites. A stacking fault grain 
boundary is where two such islands with different stackings meet each other. Figure 4.2 gives an example of a stacking fault grain boundary.

The step edges of islands on a (111) facet are of two types: A-steps and Bsteps. In an A-step the step edge atoms meet above gaps between the atoms in the layer below. The edge atoms in B-steps meet on top of atoms in the layer below. In Figure 4.3 we show examples of hexagonal FCC and HCP stacking islands and their corresponding A-steps and B-steps. These A-steps and B-steps alternate around these islands. The atoms in FCC and HCP islands sit on different sets of sites, so the pattern of A-steps and B-steps is reversed between these FCC and HCP islands.

When an FCC and an HCP island meet along A-step edges, this stacking fault grain boundary is called an A-gap. Similarly when alternate stacking islands meet along B-step edges, the stacking fault grain boundary is called a B-gap. Because of the reversal in the pattern of A-steps and B-steps between FCC and HCP stacking islands, an A-step on one island cannot meet with a B-step on an alternate stacking island, preventing coalescence of the islands.

Note that a triangular shaped island will be composed of either all A-steps or all B-steps. But even in these cases, because of the reversed orientation of such triangular islands on alternate stacking, they are limited to forming A-gaps or Bgaps when they meet. Figure 4.4 shows stacking fault grain boundaries exhibiting A-gaps and B-gaps. 


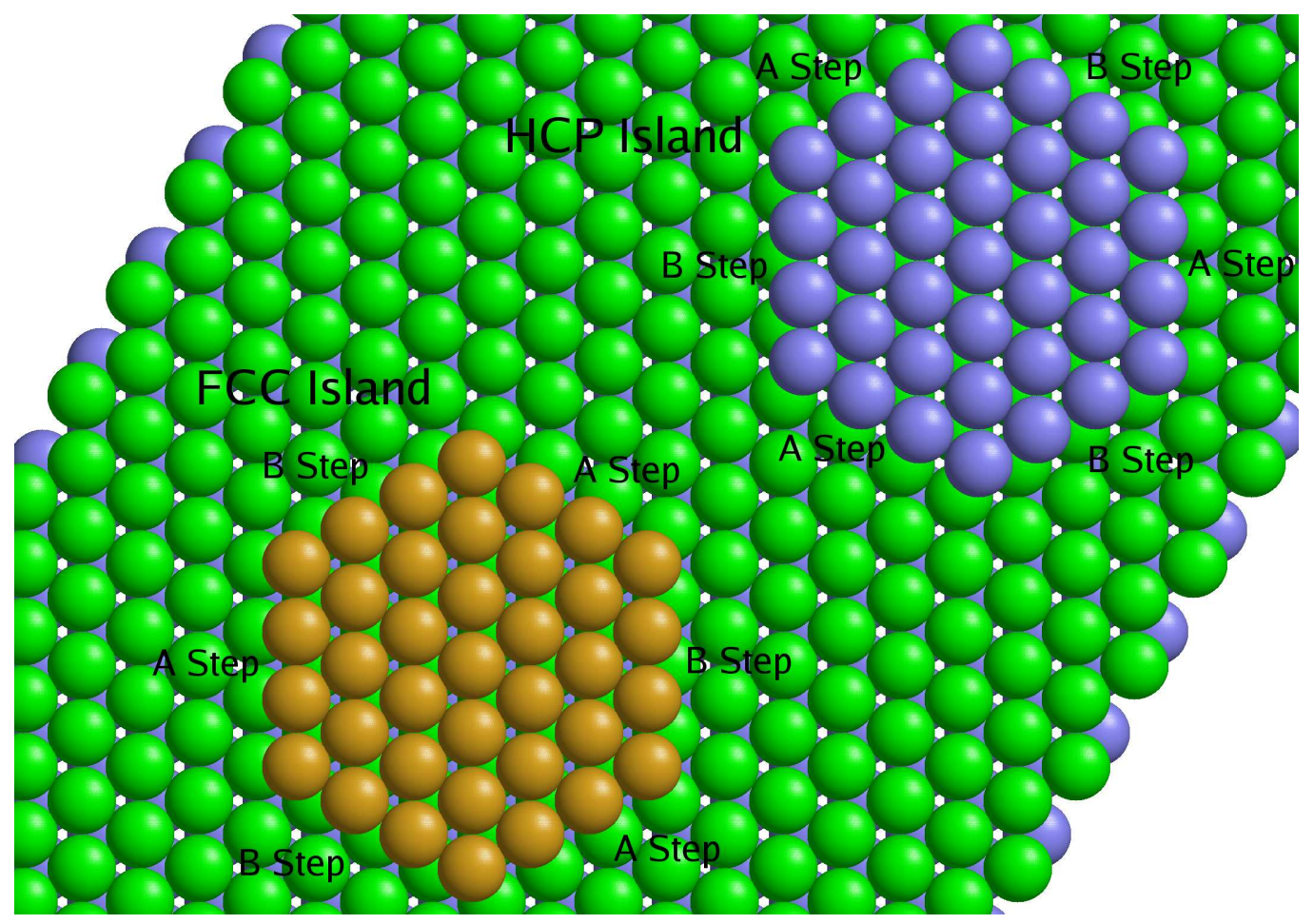

Figure 4.3: This figure shows two hexagonal mono-layer islands on a (111) facet. The atoms are coloured gold in the FCC stacking island and blue in the HCP stacking case. The alternating A-steps and B-steps at the edges of these respective islands are identified. In an A-step the step edge atoms meet above gaps between the atoms in the layer below. The edge atoms in B-steps meet on top of atoms in the layer below. 


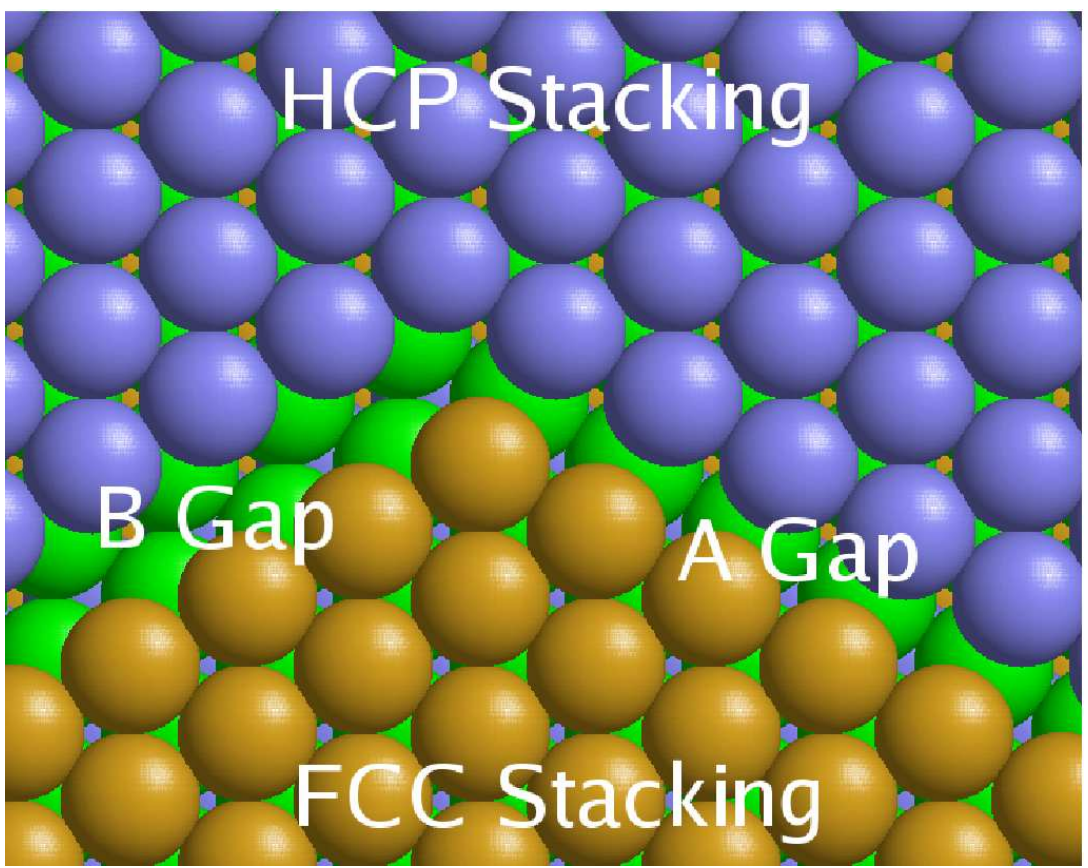

Figure 4.4: This image shows a close up view of stacking fault grain boundaries between FCC and HCP stacking regions. On the left is a B-gap (where two Bsteps meet) and on the right is an A-gap (where two A-steps meet). Atoms in a B-gap can move out of their edge into the hollow site in front of them. In this case they change to the alternate stacking. Atoms in A-gaps are head to head and do not have room to move in this way. 


\subsection{Stacking Fault Grain Boundaries}

This section describes the stacking fault grain boundaries simulated in this thesis.

\subsubsection{Slab of Atoms}

In these simulations, mono-layer FCC and HCP stacking islands were created on (111) faceted slabs of Lennard-Jones atoms. These slabs have 12 columns of atoms across their width of $10 \sigma$. They have six layers of atoms in a depth of $5 \sigma$. Slabs of different lengths were used in different cases, but the minimum length was $81 \sigma$, encompassing 144 rows of atoms. An example slab is shown in Figure 4.5 .

\subsubsection{FCC and HCP site energies}

The difference in potential energy between adatoms located on FCC and HCP sites was determined in the following way. Two slabs were constructed without any stacking fault grain boundaries. In one slab the top layer of atoms formed a single FCC stacking layer. The second slab had the top layer of atoms in HCP stacking. These slabs were relaxed using MD. The MD simulations ran for 10, $000 t$ (where $t$ is the Lennard-Jones reduced time step), with a set temperature of $T=0.0$. A Langevin thermostat with $\gamma=1.0$ was used to control the temperature. For each slab the temperature initially increased to approximately 0.006 as the atoms relaxed and then rapidly declined to 0.0. The potential energy declined and rapidly converged for each slab. For the slab with FCC stacking the potential energy per atom converged to $-7.0032 \epsilon$. In the case of the HCP stacked slab the potential energy per atom converged to $-7.0078 \epsilon$. This difference in potential energy between the two slabs is attributable to the different stacking of the atoms in the top layers. The HCP stacking slab has deeper potential wells for adatoms which implies that adatoms on HCP sites are in more stable configurations than adatoms on FCC sites. The deeper potential wells in HCP stacking is attributed to the fact that HCP threefold hollow sites have a second neighbour atom directly below. We 
conclude that for the atomic slab geometry used in this work, HCP stacking is favoured over FCC stacking, as HCP has the lower potential energy.

\subsubsection{Initial Configuration}

The initial configuration of a slab of atoms comprises a pair of HCP/FCC stacking fault grain boundaries positioned at approximately $25 \%$ and $75 \%$ of the length slab. These grain boundaries lie straight across the width of the slab as seen in Figure 4.6. They are composed of alternating A-gaps and B-gaps, each of which is only one atom spacing in extent.

This configuration provides a central island in either FCC or HCP stacking, impinging on a pair of alternate stacking islands at either end of the slab. These apparently separate end islands are in fact in contact with each other by way of the periodic boundary conditions applied along the length of the slab. Hence, for consistency, islands at both ends of the slab must have the same stacking, requiring the placement of a pair of grain boundaries on the slab. Periodic boundary conditions also apply across the width of the slab to maintain the atoms in this slab geometry.

The atoms are colour coded to identify which stacking, FCC (Gold) or HCP (Blue), applies to each part of the slab. This colour coding enables changes in the location and extent of the stacking islands to be followed visually during a simulation.

A slab with an FCC stacking island in its center and HCP islands on its ends is shown in its initial configuration in Figure 4.5. Both top and side views are shown.

\subsection{Grain Boundary Shape}

The changes in the shape of grain boundaries during the simulation are discussed in this section. Once the simulation begins, visual inspection shows that the shape 

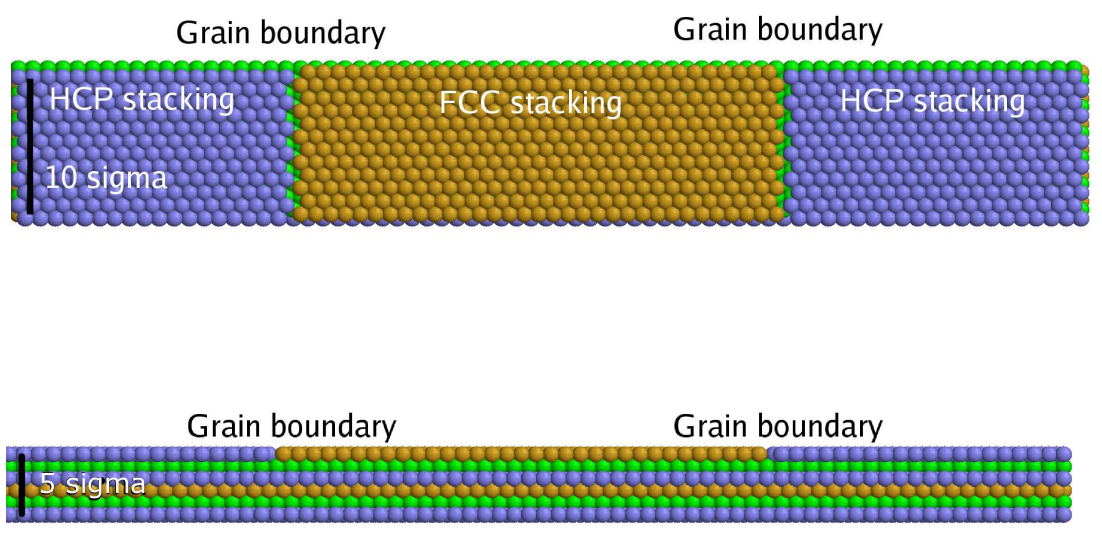

81 sigma

Figure 4.5: Top view and side view of a slab of atoms with a central FCC stacking island.

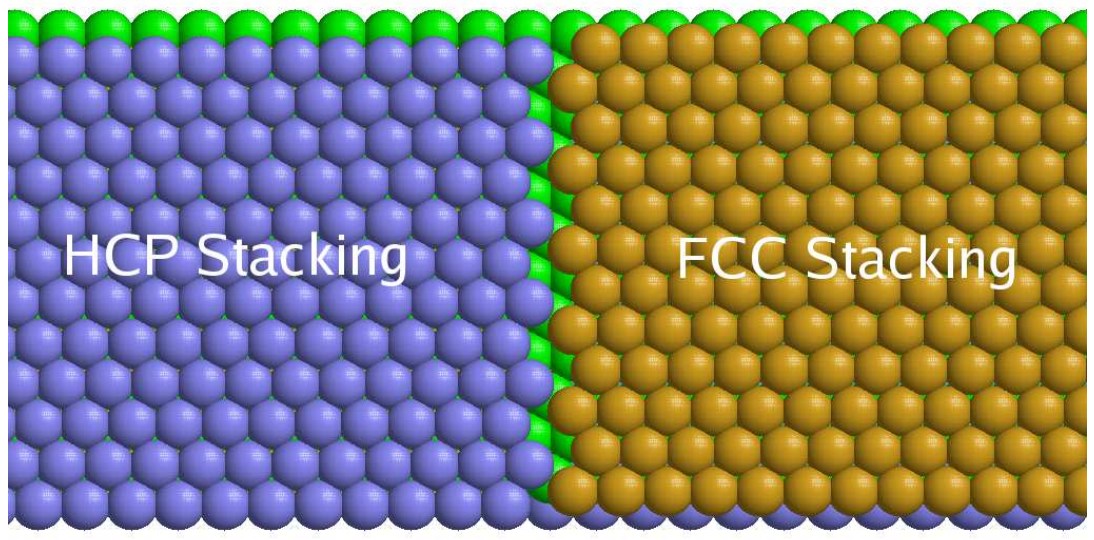

Figure 4.6: The center of this image shows a grain boundary in its initial configuration, straight across the width of the slab. 
of grain boundaries becomes more complicated, changing from the initial straight line to mixtures of more extensive A-gaps and B-gaps, as demonstrated in Figures 4.7 and 4.8. As the simulation progresses these mixtures continually change as atoms shift back and forth across the grain boundary between the different stacking islands. In Figure 4.7 and Figure 4.8 we see some examples of the different shapes the grain boundaries may take at different times.

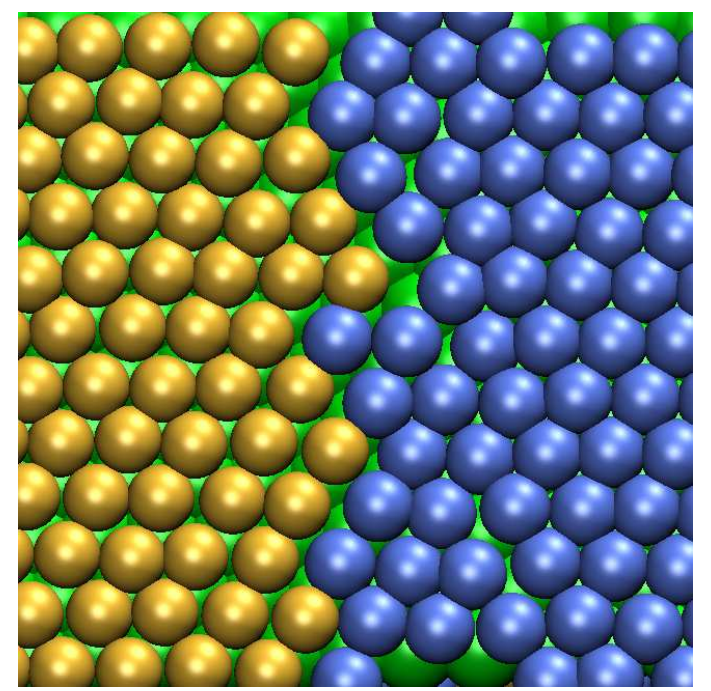

Figure 4.7: This grain boundary is composed mainly of B-gaps, where atoms on the different stacking islands are offset with respect to each other giving them room to move and the opportunity to cross the grain boundary. 


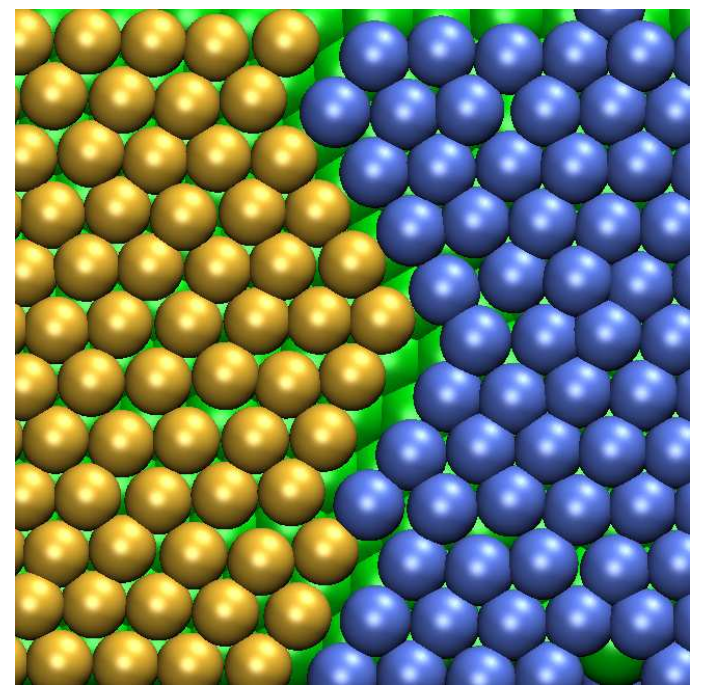

Figure 4.8: In this chevron shaped grain boundary, the atoms in the upper arm are mostly in an A-gap configuration "head to head" and so are unable to move. However the atoms in the lower arm are in a B-gap arrangement which allows them to readily move.

Due to the periodic boundary conditions and the narrow width of the slab, the curvature of the grain boundaries is limited. During the simulations the extent or spread of grain boundaries along the length of the slab was recorded. This spread of a grain boundary is referred to as the width of the grain boundary in this work. The width was determined from the number of rows of atoms along the length of the slab that contained some atoms in FCC sites and some in HCP sites. The initial straight line configurations change to mixtures of A-gaps and B-gaps, making the grain boundaries more convoluted and increasing their width. The mix of A-gaps and B-gaps, and hence the width of the grain boundaries, varies during a simulation. From MD simulations the average width of a grain boundary was found to be $2.8 \sigma$, with a maximum width of $7.3 \sigma$. This average is taken over 18 grain boundaries from 9 simulations of duration $10000 t$ each. The maximum is the greatest width measured in the 9 simulations. The changes in width of a grain boundary during a simulation is shown in Figure 4.9. 


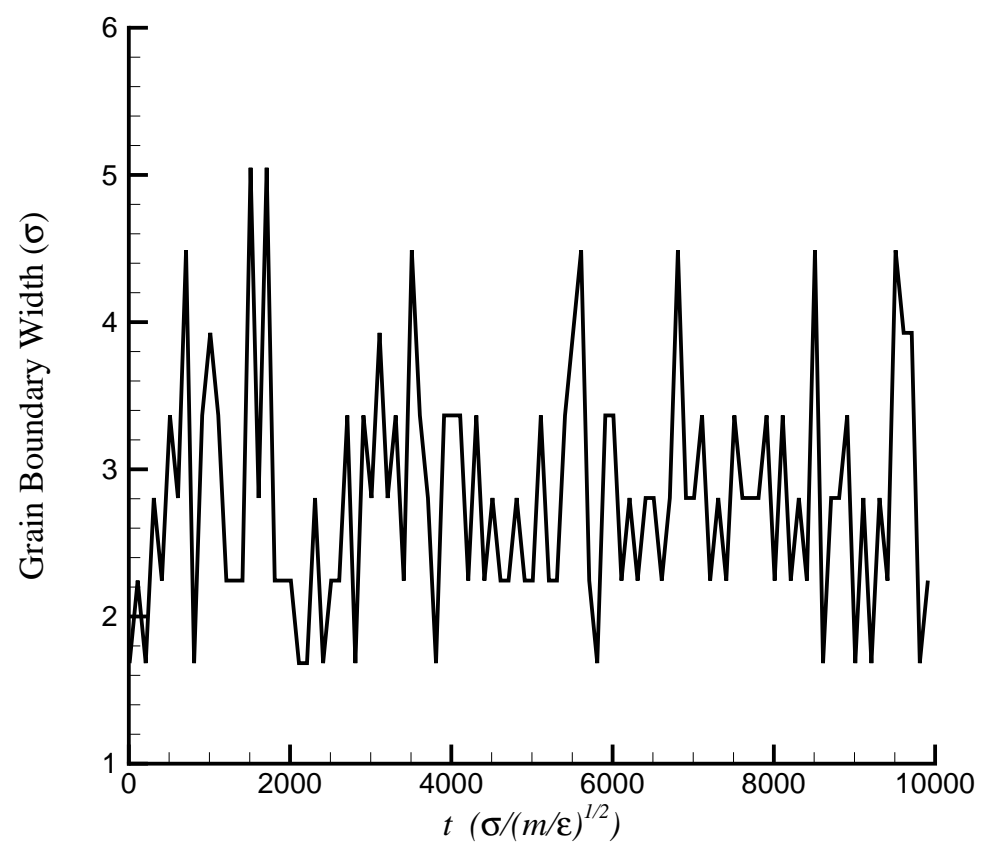

Figure 4.9: The changing width of a single grain boundary is graphed over the 10000 time steps of a simulation. Width values at every $100 t$ are shown. The width generally remains within the range $2.3 \sigma$ to $3.4 \sigma$, with occasional fluctuations to larger or smaller values. There is no obvious trend in width during the simulation.

The orientation of the A-gaps and B-gaps in the grain boundaries follows the "natural directions" on the 111 facet, as indicated on the grain boundary in Figure 4.10. 


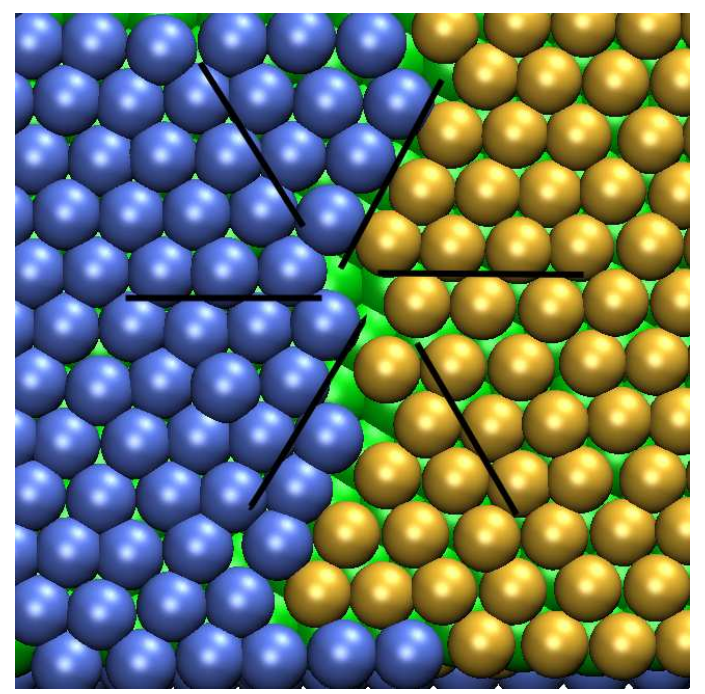

Figure 4.10: The black lines indicate the natural horizontal and diagonal directions on the (111) facet, due to the triangular shape of the lattice.

The existence of these directions is conducive to the formation of triangular shaped stacking fault islands with opposite orientations for FCC and HCP stacking. Instances of such islands are shown for Iridium islands on an Iridium (111) surface in the image from Busse et al. [146] in Figure 4.11. 


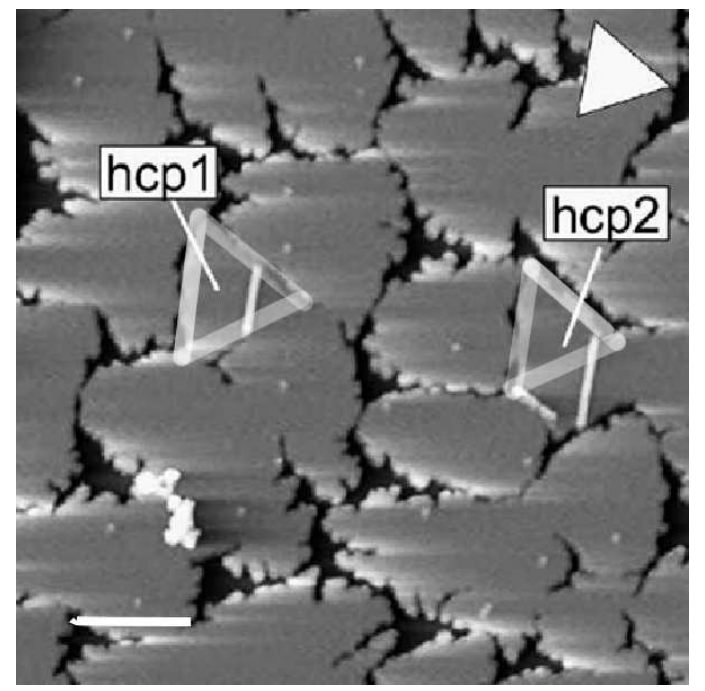

Figure 4.11: Image (used with permission) from Busse et al. [146], showing triangular stacking fault islands of Iridium on Iridium. The white triangle gives the orientation of HCP stacking islands. Two HCP islands are delineated by translucent white lines. Islands with the opposite orientation are in FCC stacking. The white scale bar at the bottem left is $100 \AA$ long.

In some instances a grain boundary can attain a zigzag configuration as shown in Figure 4.12.

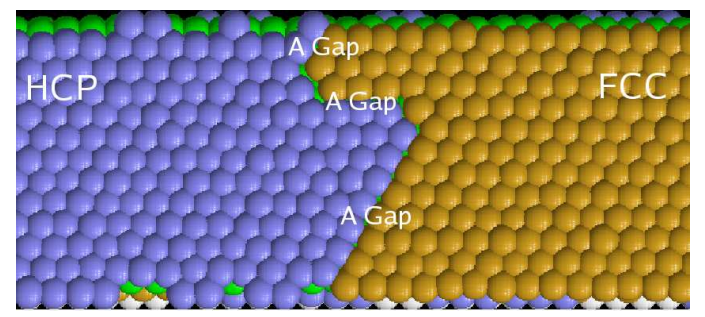

Figure 4.12: This grain boundary has evolved into the zigzag conformation. The atoms along both sides of the diagonals and the horizontal of the zigzag meet their counterparts in the other stacking island in A-gap configurations. These atoms are so closely packed that they are unable to change their local configuration (without climbing out of the surface epitaxial layer). Only the atoms at the apexes of the zigzag, which are in B-gap configurations, have sufficient room to readily move. 
This is an attempt at the formation of a pair of impinging triangular stacking fault islands. Such zigzags have a large proportion of A-gaps which are comprised of closely packed atoms which do not have space to cross over to the other stacking island. Consequently once formed, a grain boundary may remain in such a configuration for some time.

A grain boundary tends to approximate the initial shape when its width is very narrow, while the zigzag shape as in Figure 4.12, typically occurs when the width is long. Most of the time the grain boundary is of medium width, as shown in Figure 4.9, and then its shape is usually composed of a mix of A-gaps and B-gaps, as seen in Figures 4.8 and 4.10 for example.

The change in grain boundary shape can be a relatively rapid process. The zigzag configuration in Figure 4.12 was formed within $50 t$ of the start of the simulation.

Over a longer time scale the average positions of the grain boundaries move along the slab.

\subsection{Atomic Details of Grain Boundary Motion}

The observed grain boundary motion is the aggregate result of the atoms on either side of the grain boundary repeatedly crossing the boundary (enlarging one stacking fault island at the expense of the other) thus altering the configuration and position of the grain boundary over time.

In the "kink flip" move an atom crosses a B-gap in the grain boundary from one stacking island to another and adheres to a kink site on the adjacent island, thereby moving the kink site along the grain boundary by one atom. By continuing this process in a "zipper like" fashion a whole row of atoms changes from one type of stacking to the other and the position of the grain boundary is moved by one row. Figure 4.13 demonstrates this process. 

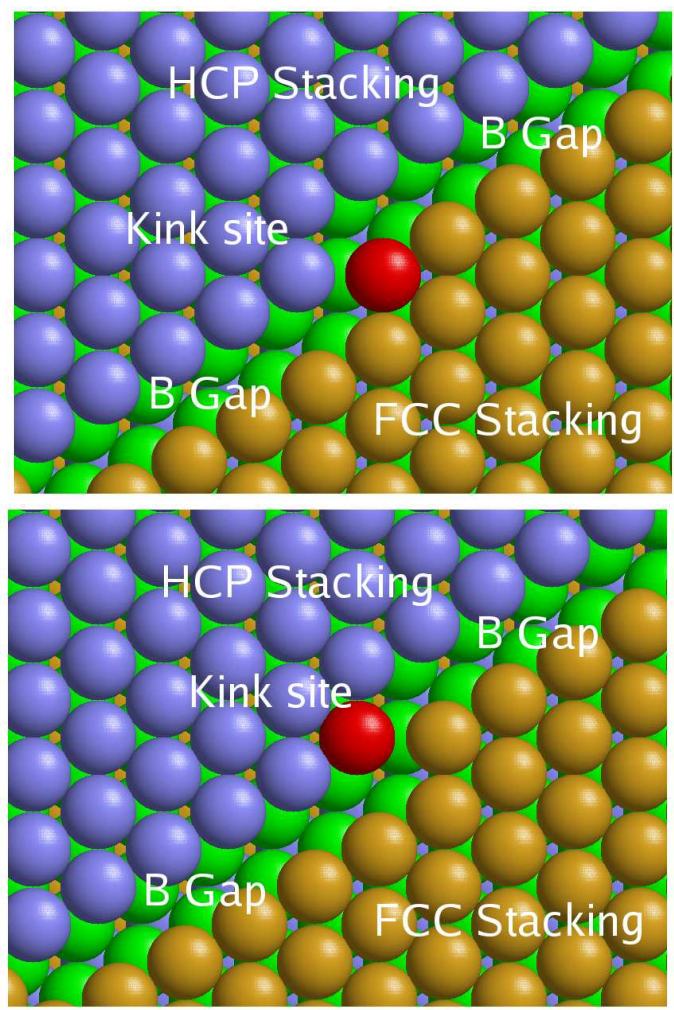

Figure 4.13: The top image shows the red atom about to make a "kink flip" move across the B-gap and join the HCP stacking island. The bottom image shows the red atom after completing the "kink flip" move. The kink site has moved along the B-gap.

When a section of a B-gap in a grain boundary does not have kink sites, it is necessary for them to be created before a sequence of "kink flip" moves can occur. In these cases kink sites are most easily produced when an atom at a corner site crosses the grain boundary. One or more positive (outward) kinks are formed on the island the atom has just moved to, while a negative (inward) kink remains on the island the atom has moved from. In Figure 4.14 we see an atom making such a "corner crossing" move and producing kink sites. Figure 4.15 shows a pink coloured atom subsequently making a "kink flip" move into one of the newly created kink sites and thus shifting part of the B-gap on the left side of the FCC 
island. The next figure, Figure 4.16 demonstrates an alternative "kink flip" move by the grey coloured atom, into the other newly created kink site. This moves part of the A-gap on the right of the FCC island.

In the case of a grain boundary being in the zigzag configuration the islands are separated exclusively by a pair of A-gaps. Figure 4.17 shows this situation. After the corner atom has made a "corner cross" move, a short B-gap (composed of two kink sites) is produced as shown in Figure 4.17 (bottom). Atoms can then cross this short B-gap by moving into the kink sites, as seen in the top image in Figure 4.18. Once that configuration has been reached, "kink flip" moves can then occur, which will result in moving part of the B-gap (sited on the left of) or Agap (sited on the right of) the FCC island. Such moves are shown in Figure 4.18 (bottom) and Figure 4.19 (bottom) respectively.

The relative frequency of atomic moves which alter the location of the grain boundaries can be assessed from the energy barriers that must be overcome for these moves to take place. The following energy barriers for these atomic moves were determined using the NEB method (described in chapter 3). These NEB calculations used a slab of atoms 21 atomic rows wide, 28 atomic rows high and 6 atomic layers deep. A chevron shaped grain boundary was formed across the center of the top layer of the slab, separating FCC and HCP stacking islands. For some cases, one arm of the grain boundary was formed by a B-gap and the other by an A-gap. In other cases both arms of the grain boundary were formed by Agaps. Atoms within a distance of $4 \sigma$ of the moving atom were free to move while those further away were fixed. Using MD the initial and final configurations were relaxed by slow cooling. Then a chain of seven intermediate configurations was interpolated between the initial and final configurations. The chain of images was then relaxed with elastic band forces applied to determine the minimum energy path between the initial and final configurations. The maximum value of the energy along the minimum energy path less the energy of the initial configuration is the energy barrier for the configurational change or atomic move. 
Table 4.1: Energy Barriers

\begin{tabular}{ccc} 
Atomic move & Figure & Energy barrier $\epsilon$ \\
\hline FCC to HCP & & \\
"kink flip" B-gap & 4.13 & 0.71 \\
"corner cross" A-gap B-gap & 4.14 & 0.72 \\
"kink flip" A B corner & 4.15 & 0.72 \\
"corner cross" A-gap A-gap & 4.17 & 0.41 \\
"kink flip" A A corner & 4.18 & 0.71 \\
HCP to FCC & & \\
"kink flip" B-gap & & 0.74 \\
"kink flip" A B corner & & 0.74 \\
"kink flip" A A corner & & 0.77
\end{tabular}

The energy barriers in Table 4.1 are all of the order $0.70 \epsilon$, except for the "corner cross" at an intersection of A-gaps. This lower energy barrier reflects that fact that the moving atom leaves a site with only two, rather than three, in-plane nearest neighbours.

Based on these energy barriers and the geometric constraint that atoms in Agaps have limited motion, the "kink flip" across a B-gap appears to be the key atomic process for grain boundary migration for the slab geometry and temperature range studied in this work.

Given its lower energy barrier, the "corner cross" at an intersection of A-gaps occurs more frequently than the "kink flip". Once such a move has occurred, the changed atomic geometry allows "kink flip" moves to occur. A series of "kink flip" moves which are (in aggregate) in the same direction can move a grain boundary by one atomic row, in which case the average observed energy barrier per move for the displacement of a grain boundary by an atomic row (due to a sequence of atomic moves) will be approximately $0.70 \epsilon$.

The energy barriers for the "kink flip" moves from HCP stacking to FCC stacking are a little higher than for the "kink flips" from FCC to HCP. This will give 
a slight preference to HCP stacking over FCC stacking during the course of a simulation.

Note that in respect of the migration of stacking fault grain boundaries in Iridium, it has been argued by Busse [146] that "kink flip" moves are the key processes.
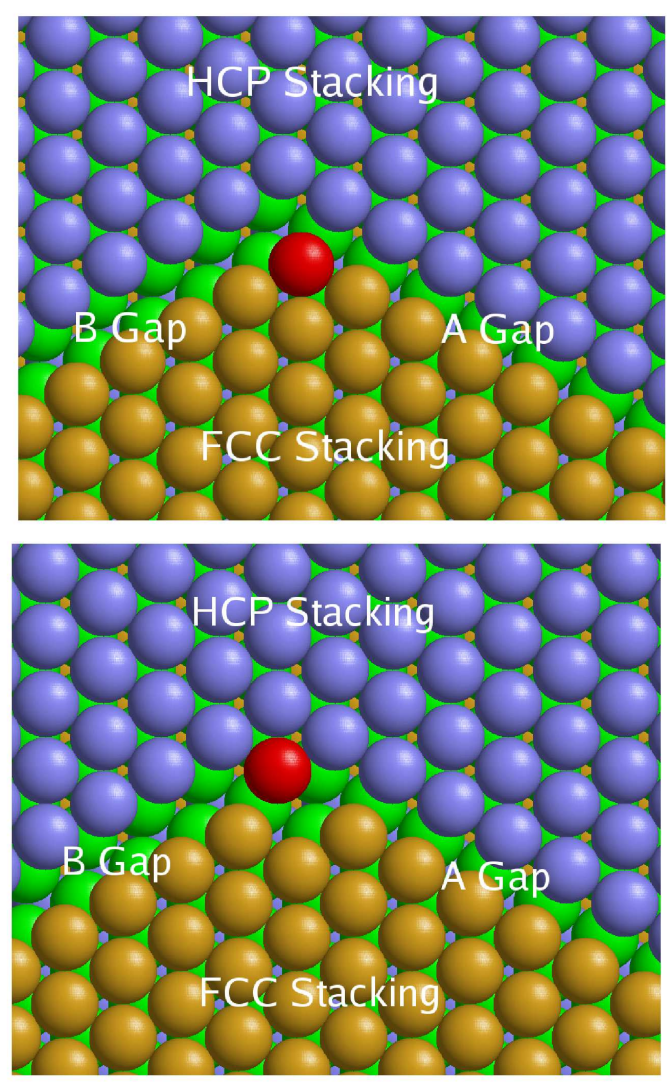

Figure 4.14: The top image shows the atom at the apex of the FCC island (coloured red) about to "corner cross" and join the HCP stacking island. In the bottom image the red coloured atom has just completed a "corner cross" move. 

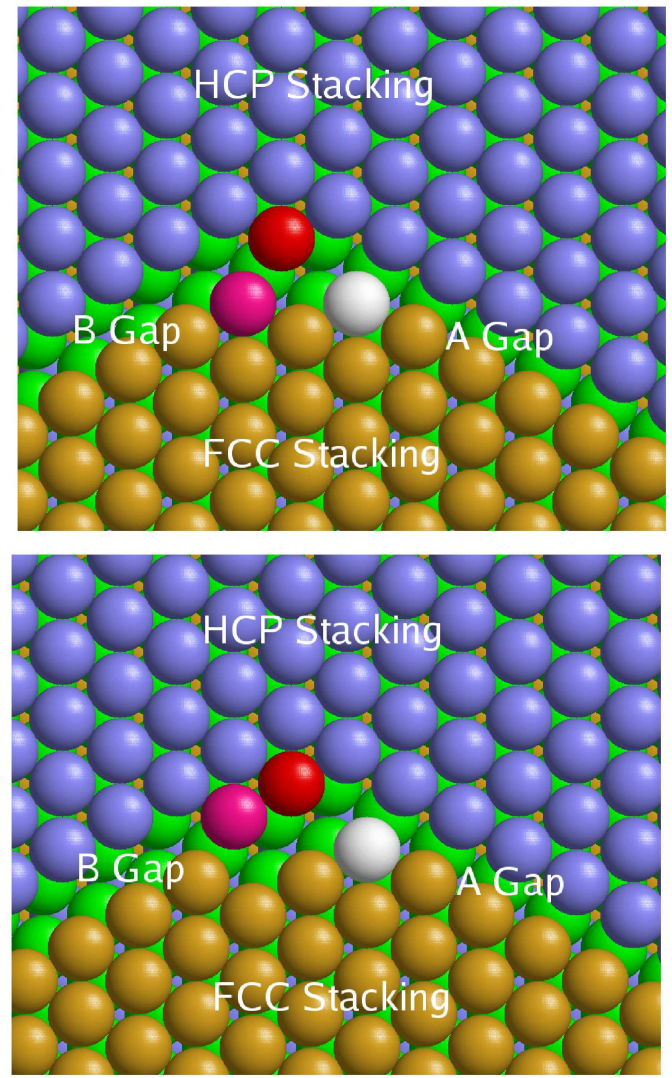

Figure 4.15: In the top image the red coloured atom has attached to the HCP stacking island creating kink sites on the HCP island. The bottom image shows the pink coloured atom after it has just completed a "kink flip" move into one of the kink sites. 

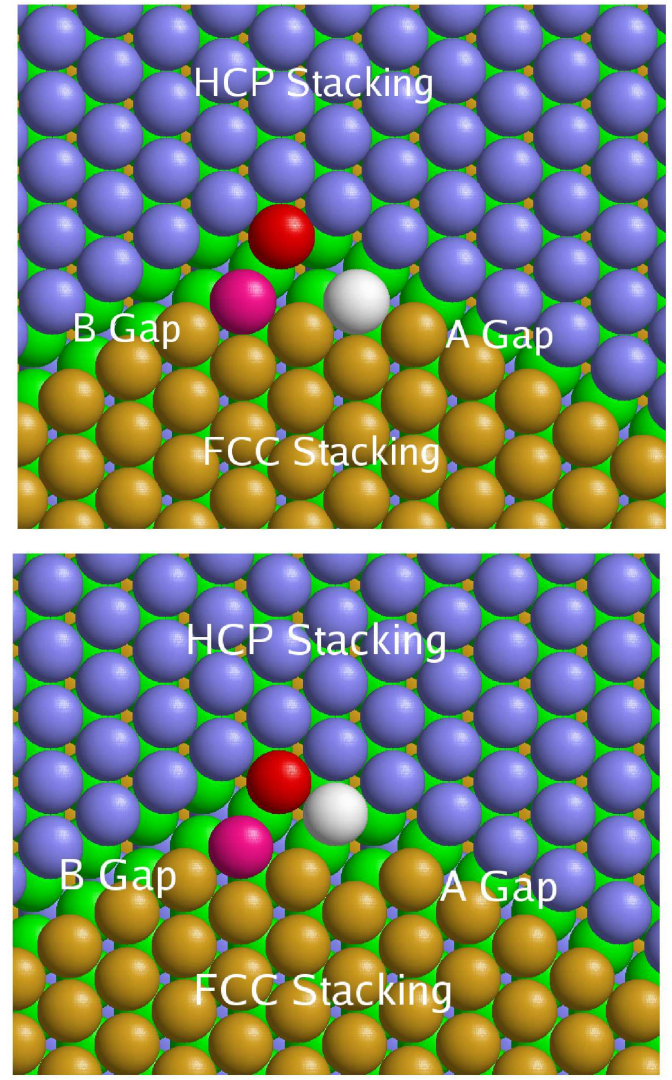

Figure 4.16: In the top image the red atom has attached to the HCP stacking island, creating kink sites on the HCP island. The bottom image shows the grey coloured atom after it has just completed a "kink flip" move, and attached itself to the HCP island. Repeated "kink flip" moves will relocate the A-gap by one atomic row. 

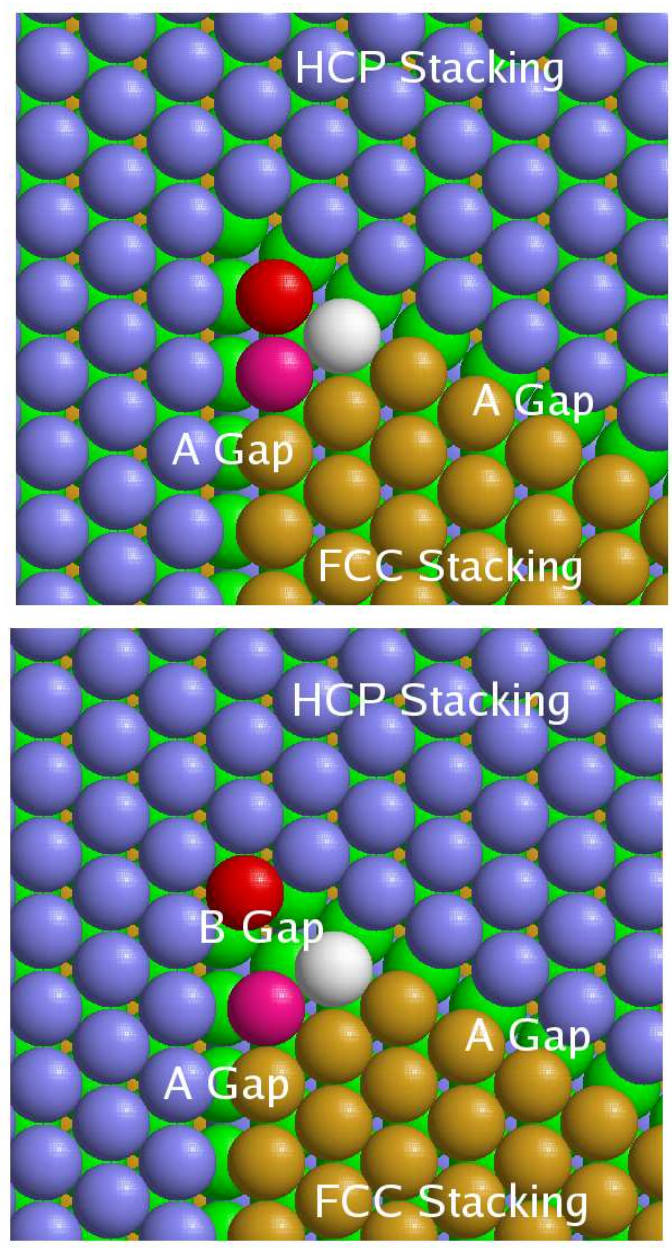

Figure 4.17: This pair of images shows a triangular FCC island meeting an HCP island producing a pair of intersecting A-gaps. The top image shows the red atom about to "corner cross" from the FCC island to the HCP island. The lower figure shows the short B-gap formed after the red atom has attached to the HCP island. 

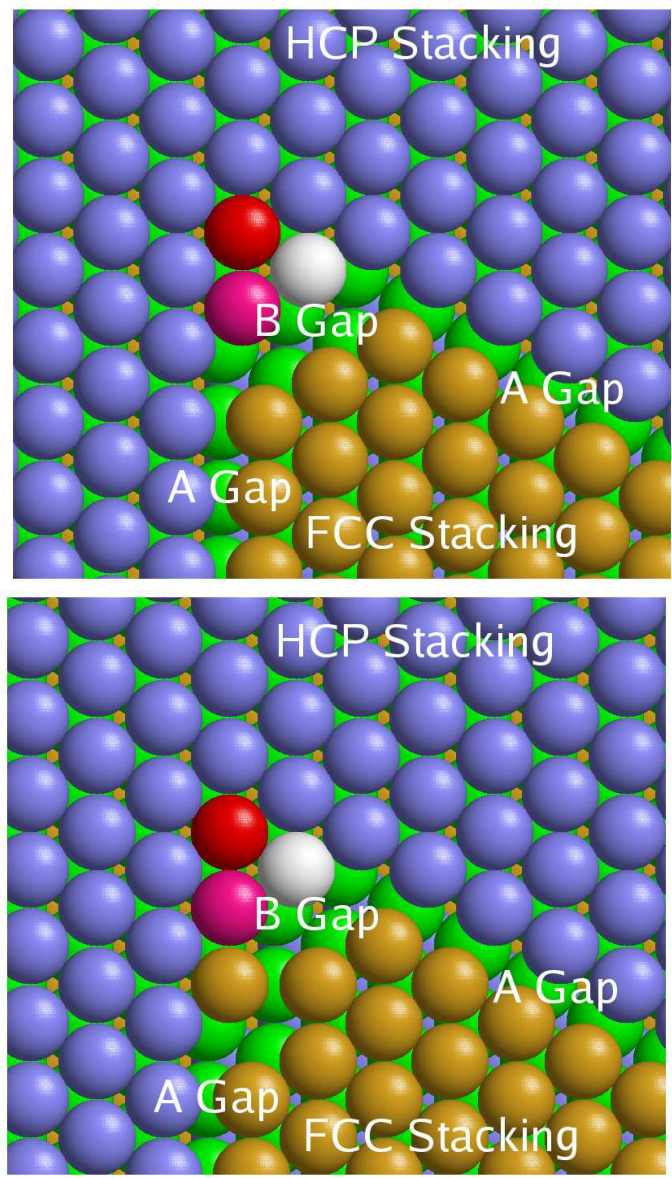

Figure 4.18: In this figure the top image shows a short B-gap formed after the red, grey and pink coloured atoms have moved from the FCC island and become part of the HCP island. In the lower image a gold coloured atom has made a "kink flip" move across the B-gap at the apex of the FCC island and has attached itself to the HCP island. 

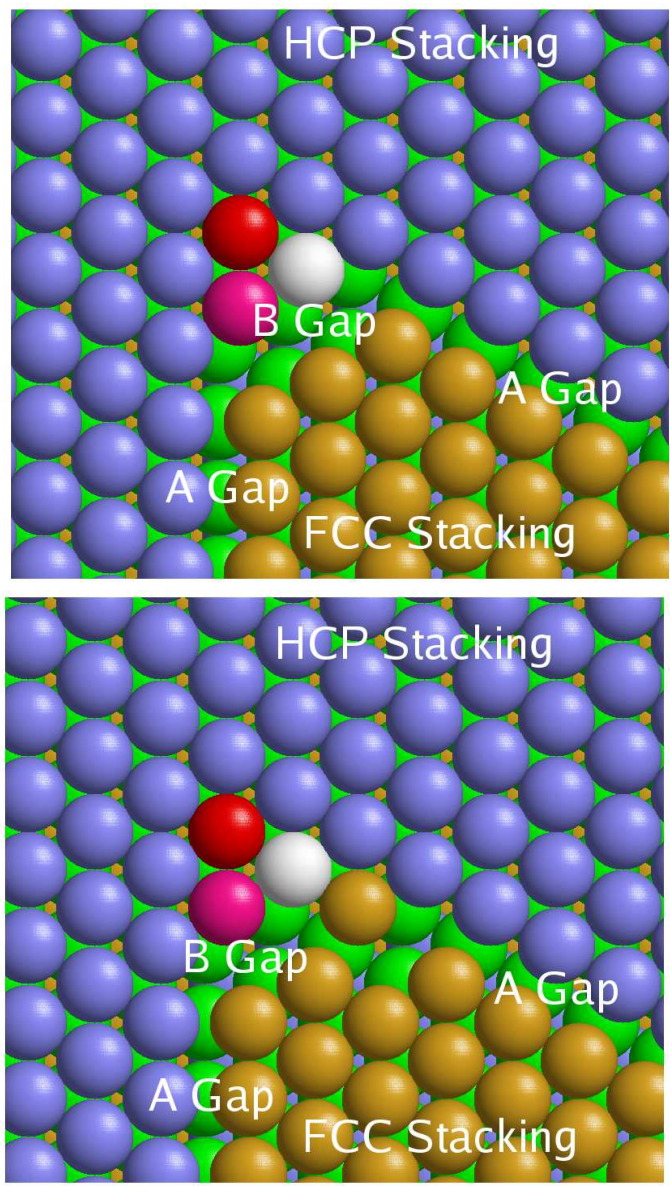

Figure 4.19: The top image in this figure also shows a short B-gap formed after the red, grey and pink coloured atoms have moved from the FCC island and become part of the HCP island. In the lower image a gold coloured atom on the right hand side of the FCC island has made a "kink flip" move across the B-gap at the apex of the FCC island and joined the HCP island. 


\subsection{Grain Boundary Migration}

\subsubsection{Changes in Grain Boundary Position}

At each time step the position of a grain boundary was determined as the average position of the rows of atoms along the length of the slab that contained a mixture of atoms in FCC sites and HCP sites. During a simulation run the positions of the grain boundaries along the length of the slab vary. Atoms cross back and forth, attaching to and detaching from the two different stacking islands. A series of "kink flip" moves can "zipper" along a grain boundary and move it by one atomic row. A sequence of such one row moves in the same direction will enlarge one island at the expense of the other and reposition the grain boundary sited between them.

Figure 4.20 demonstrates such a migration of grain boundary positions over time. In each simulation run a distinct random seed is used to provide unique random velocities to all the atoms, consistent with the chosen temperature for the simulation. In this way each simulation run provides a distinct instance of grain boundary behaviour. It was found that the detailed motion of the grain boundary positions can differ significantly between separate simulation runs at the same temperature, from the extensive motion as shown in Figure 4.20 to very limited motion, and in some cases meeting and annihilating each other due to the periodic boundary conditions. The migrations are composed of periods of stability or steady progression, interspersed with occasional rapid excursions. 


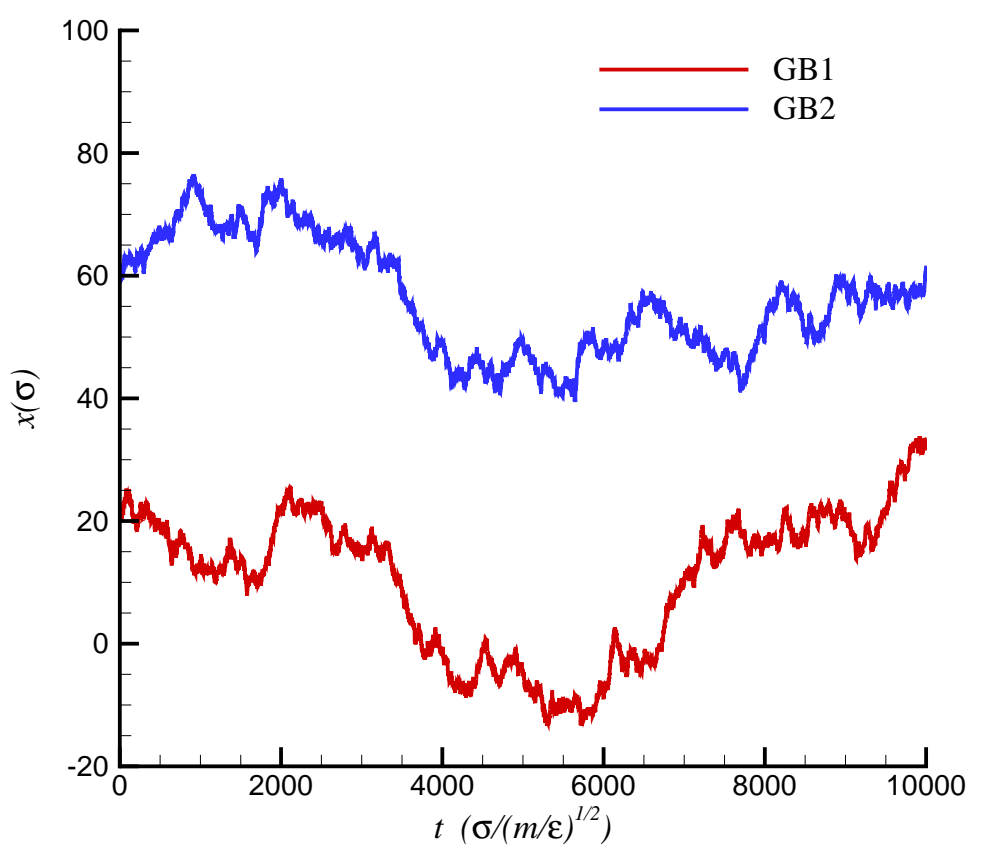

Figure 4.20: This graph is a time trace of the migration of the two grain boundaries over the surface of the slab during a simulation. The slab is located along the yaxis of the graph, with the lower grain boundary (GB1) initially positioned at $25 \%$ of the length of the slab and the upper grain boundary (GB2) initially positioned at $75 \%$. There is considerable rapid short range motion which makes the graph appear as a broad band approximately $5 \sigma$ thick. Over a longer time period the grain boundaries can migrate across a substantial proportion of the surface of the slab. Because periodic boundary conditions are applied, grain boundaries reaching the end of the slab are able to "wrap around". Here the graph of GB1 has been unwrapped and extended beyond the end of the slab (hence the negative range for $\sigma)$.

\subsubsection{Random Walk}

For simulations at temperature $T=0.35$, it was found that the grain boundary migration could be quantified in terms of the mean square displacement (MSD) of 
the grain boundary position

$$
\left\langle\Delta x(\Delta t)^{2}\right\rangle=\left\langle(x(t+\Delta t)-x(t))^{2}\right\rangle
$$

in a time interval $\Delta t$. The MSD curves differ significantly between individual grain boundaries. However when the MSD curves of 30 individual grain boundaries were averaged, a clear linear trend emerged, as seen in Figure 4.21. The diffusion coefficient $D$ for two dimensional motion is related to the mean square displacement by

$$
D=\frac{\left\langle\Delta x(\Delta t)^{2}\right\rangle}{4 \Delta t}
$$

The linear trend (constant slope) in the averaged MSD curve indicates that grain boundary migration is a diffusive process with $D=0.016$. That is, the grain boundaries appear to be performing a simple random walk over the surface of the slab. 


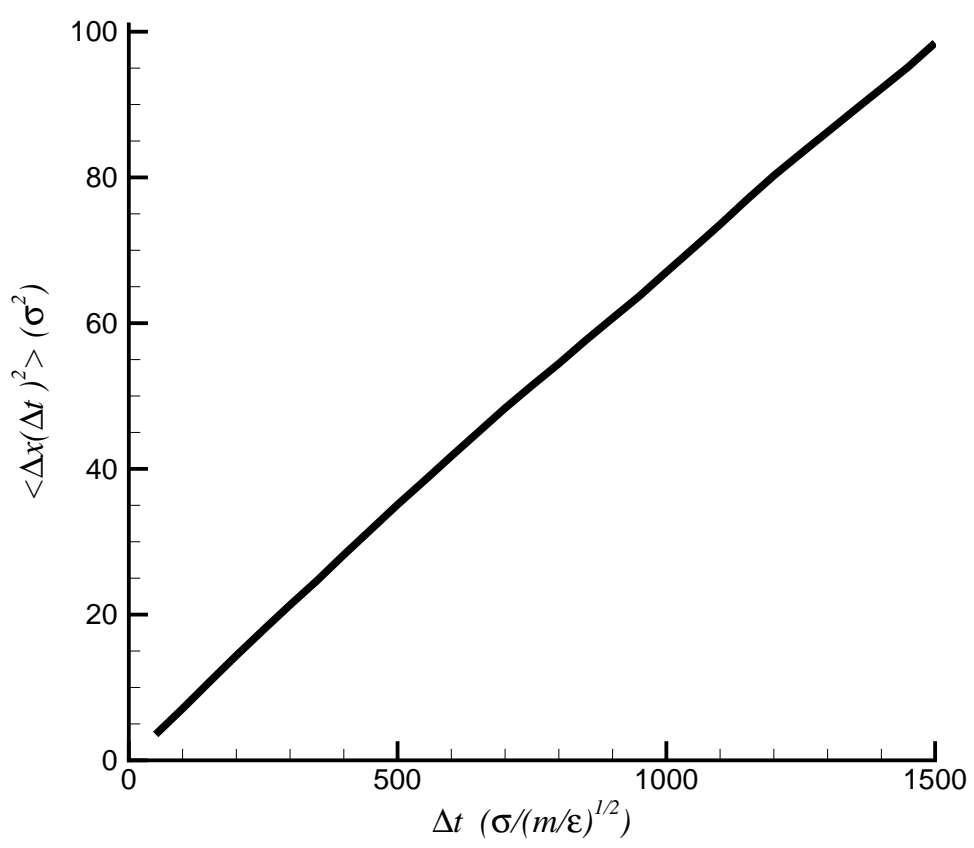

Figure 4.21: The average grain boundary mean square displacement $\left.\left\langle\Delta x(\Delta t)^{2}\right\rangle\right)$ versus $\Delta t$ from MD simulations. This linear trend implies that the grain boundaries are are essentially following a diffusive random walk.

\subsubsection{Temperature Dependence}

The rate of grain boundary migration is found to be temperature dependent. Figure 4.22 shows grain boundary MSDs averaged over a number of MD simulations, at a range of temperatures $T: 0.35,0.40,0.45$ and 0.50 . In each of these cases, $\left\langle\Delta x(\Delta t)^{2}\right\rangle$ shows a distinct linear trend with $\Delta t$. Higher temperatures produce a more rapidly diffusing grain boundary, because energy barriers for atoms shifting to another site are more likely to be surmounted at higher temperatures. The respective diffusion coefficients are given in Table 4.2. 


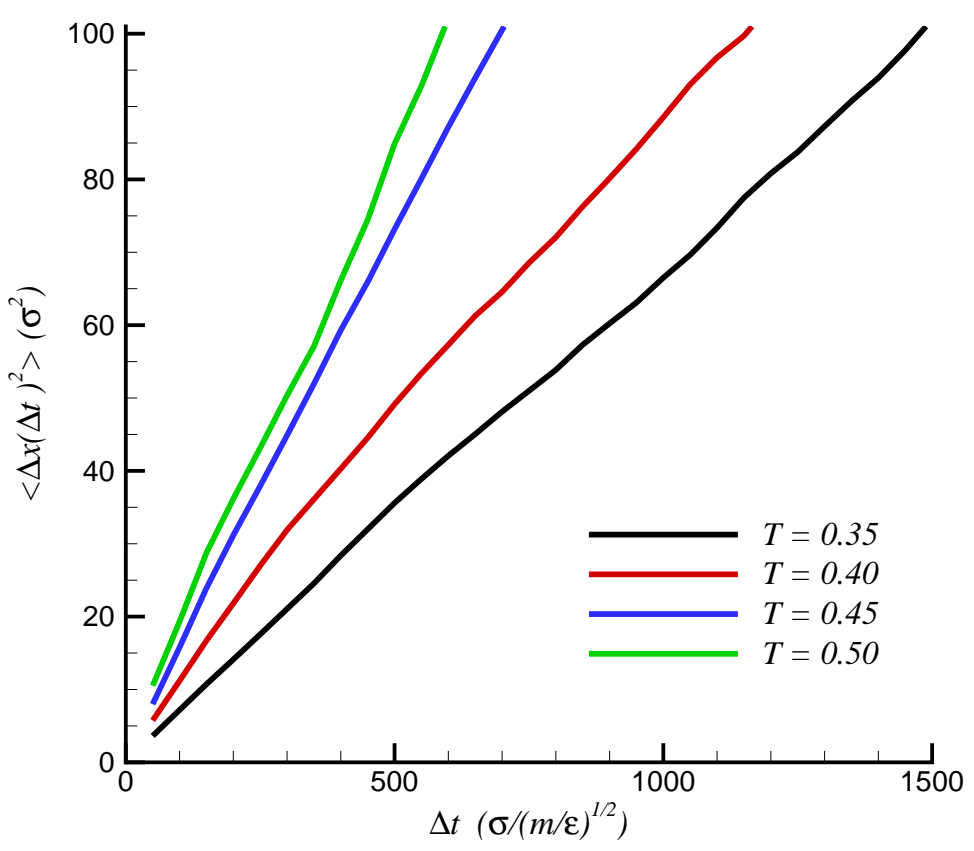

Figure 4.22: This shows the average grain boundary mean square displacement $\left.\left\langle\Delta x(\Delta t)^{2}\right\rangle\right)$ versus $\Delta t$ for various temperatures from MD simulations. The linear relationship indicates that the motion is diffusive. The Diffusion coefficient (which is proportional to the slope of graph) increases with the simulation temperature.

Table 4.2: Diffusion coefficient as a function of Temperature.

\begin{tabular}{cc}
$T$ & $D$ \\
\hline 0.35 & 0.016 \\
0.40 & 0.022 \\
0.45 & 0.037 \\
0.50 & 0.046
\end{tabular}




\subsubsection{Grain Boundary Collision}

The pair of grain boundaries on the slab are initially separated by $50 \%$ of the length of the slab. Over time they may move closer together as a result of grain boundary migration. In the case where both grain boundaries collide, the stacking fault defect between the pair of islands is eliminated and the whole surface of the slab then comprises a single stacking layer. From 31 independent MD cases of duration $10000 t$ at temperature $T=0.35$, the grain boundaries collided and the stacking fault was eliminated in 13 cases. The elapsed time until collision varied widely, from $2100 t$ to $9500 t$. When stacking faults had been eliminated in this way, there were no instances in any of the simulations of spontaneous reformation of stacking fault grain boundaries from a single stacking island.

\subsubsection{Drift}

The positions of grain boundaries on the slab may exhibit some systematic drift, as suggested by the motion of grain boundary two in Figure 4.24, in addition to random diffusion along the length of the slab. A cause of such drift may be the slight preference for HCP stacking islands over FCC stacking islands, (subsection 4.3.2). The slab in these simulations has a central FCC island with HCP islands at either end, and so growth of the islands at the ends of the slab is favoured at the expense of the central island. This stacking preference is independent of the distance between the grain boundaries.

We now show that when drift is present, the MSD will display some nonlinearity. In the case of drift as a simple linear function of time, let $b$ be the drift coefficient and $\Delta x_{r w}(\Delta t)$ the displacement for a purely diffusive random walk (one without any drift). Then the displacement of the grain boundary position with drift $\Delta x_{d}(\Delta t)$ at time separation $\Delta t$ can be expressed as:

$$
\Delta x_{d}(\Delta t)=\Delta x_{r w}(\Delta t)+b \Delta t
$$

thus

$$
\Delta x_{d}(\Delta t)^{2}=\Delta x_{r w}(\Delta t)^{2}+2 \Delta x_{r w}(\Delta t) b \Delta t+b^{2} \Delta t^{2}
$$


and since

$$
\left\langle\Delta x_{r w}(\Delta t)\right\rangle=0
$$

we have

$$
\left\langle\Delta x_{d}(\Delta t)^{2}\right\rangle=\left\langle\Delta x_{r w}(\Delta t)^{2}\right\rangle+\left\langle b^{2} \Delta t^{2}\right\rangle .
$$

Therefore when there is linear drift as well as random diffusion, the MSD will show some parabolic curvature upwards.

In Figure 4.23 we see a comparison at $T=0.35$ between the average MSD of all grain boundaries from a set of simulations and the average MSDs for the lower and the upper grain boundaries on each slab separately. The average MSD for all grain boundaries is seen to be well approximated by a diffusive random walk with slope 0.066. Also we note that at large time separations the MSDs of the upper and lower grain boundaries diverge from the MSD for all cases. These deviations suggest some drift in grain boundary positions at large time separations. However we must keep in mind that the aggregate MSD is formed as the average of the MSDs for the upper and lower grain boundaries, and so must lie between them.

We can assess the size of the drift coefficients for the upper and lower grain boundaries by fitting quadratic polynomials to their MSDs. In both cases the drift coefficients were found to be very small. For grain boundary one in Figure 4.23 the polynomial had a linear term (slope) coefficient of 0.072 and a coefficient of $-6.1 \times 10^{-6}$ for the quadratic term. This negative sign for the quadratic coefficient i.e. $b^{2}$, is inconsistent with the linear drift model. The polynomial fit to grain boundary two had a linear coefficient of 0.068 and a coefficient of $9.4 \times 10^{-7}$ for the quadratic term, giving $b=0.001$. These fitted polynomials had $r^{2}=0.99$, indicating that $99 \%$ of the variance in the MSDs is explained by the fitted polynomials. The result for grain boundary two is consistent with a small linear drift component in the random walk.

The time traces of the average positions of the two grain boundaries shown in Figure 4.24 suggest a slight drift of the grain boundaries toward each other over long time periods. As mentioned earlier, this drift could be due to the slight preference for HCP stacking islands over FCC stacking islands. 


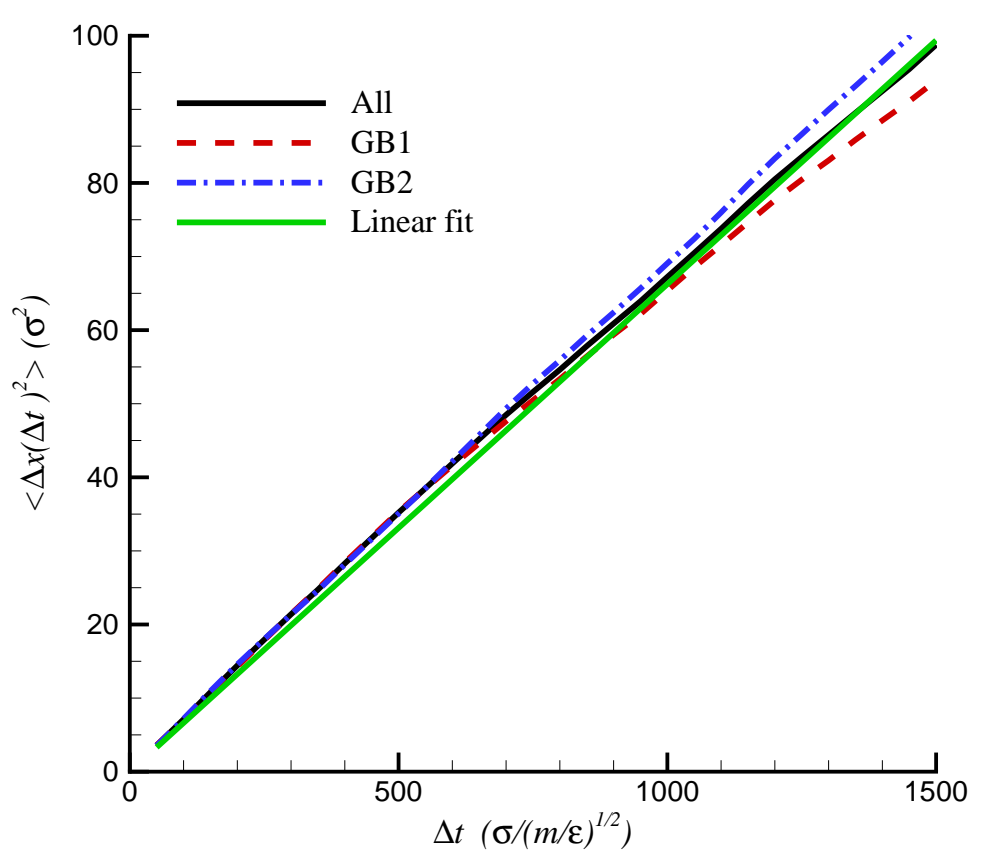

Figure 4.23: In this figure the average grain boundary mean square displacement $\left\langle\Delta x(\Delta t)^{2}\right\rangle$ versus $\Delta t$ is shown for three cases. The cases are: all grain boundaries (black) in aggregate, the lower grain boundaries (GB1) only (red) and the upper grain boundaries (GB2) only (blue). These results are from 18 MD simulations at temperature $T=0.35$ in which the grain boundaries did not merge during the $10,000 t$ duration of the simulations. A linear fit, plotted in green, has been made to the all grain boundaries data. The slope of this linear fit, 0.066 is proportional to the diffusion coefficient of the grain boundaries at this temperature. 


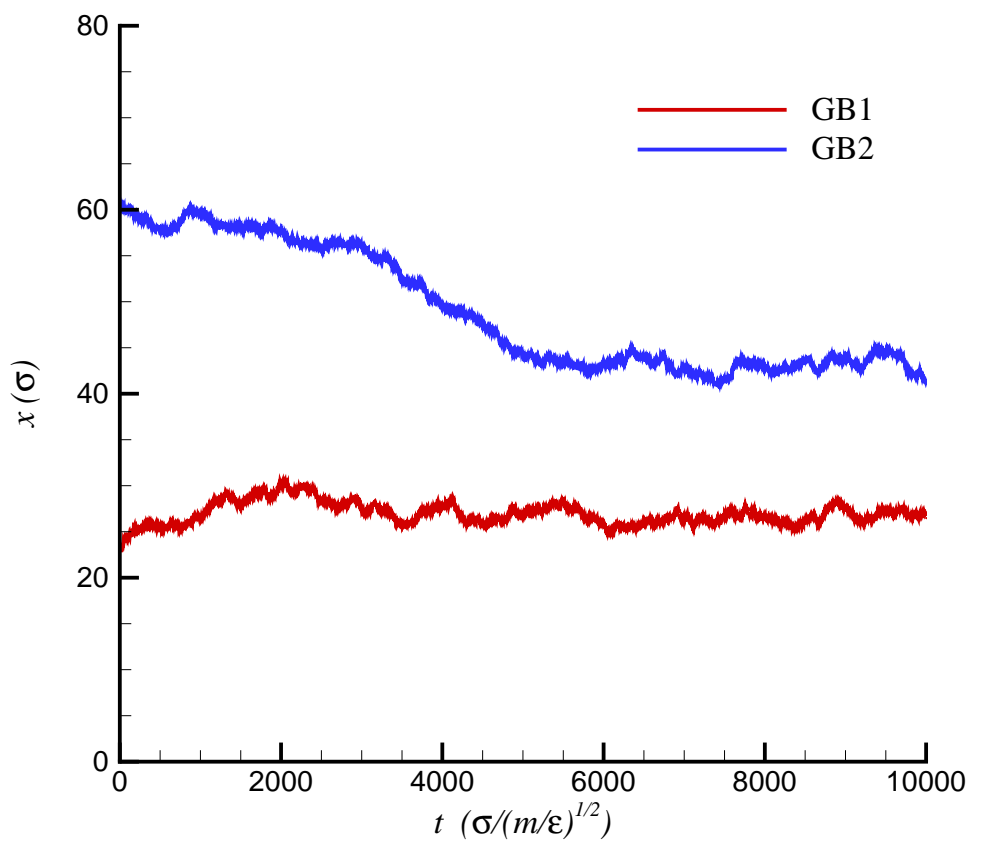

Figure 4.24: This figure shows time traces of the average positions of GB1 (red) and GB2 (blue). At each time step, the average positions of each of GB1 and GB2 are determined by averaging the positions of the respective grain boundaries from the 18 cases in Figure 4.23. Note that in these cases, the grain boundaries did not collide during the simulations. The average position of GB1 shows no significant change. GB2 trends downwards over time.

\subsection{Occurrence of Atomic Rearrangements}

We have undertaken extensive MD simulations of grain boundary migration over a range of temperatures, allowing us to identify the rate limiting step in boundary diffusion. For each of the temperatures $T=0.30$ to $T=0.45$, in steps of 0.05 , the average mean squared displacement of grain boundary motion was determined based on 24 to 28 grain boundaries at each temperature. At each temperature a regression line was fitted to the mean squared displacement graph. 
When the grain boundary diffusion follows a thermally activated Arrhenius process we have

$$
D=A e^{-\frac{\Delta E}{k_{B} T}}
$$

This can be rearranged (as $k_{B}=1$ in reduced units) to

$$
\ln D=\ln A-\Delta E \frac{1}{T} .
$$

Thus in such cases there is a linear relation ship between $\ln D$ and inverse temperature, with the negative of the slope giving the activation energy.

Figure 4.25 shows an Arrhenius plot, i.e. $\ln D$ vs $1 / T$, of the grain boundary diffusion coefficient $D=\left\langle\Delta x(\Delta t)^{2}\right\rangle / 4 \Delta t$, which demonstrates a linear relationship. The slope of the line gives an energy barrier of $\Delta E=0.70 \epsilon$, which presumably corresponds to that of the rate limiting step in grain boundary diffusion.

As expected, this is very close to the NEB calculated energy barrier for the "kink flip" move in section 4.5, supporting the hypothesis that the "kink flip" move is the key mechanism for grain boundary migration over the range of parameters studied. That is, for constant temperature situations with temperatures in the range $T=0.30, \ldots 0.45$, without deposition of adatoms and without significant multiple-atom concerted moves. 


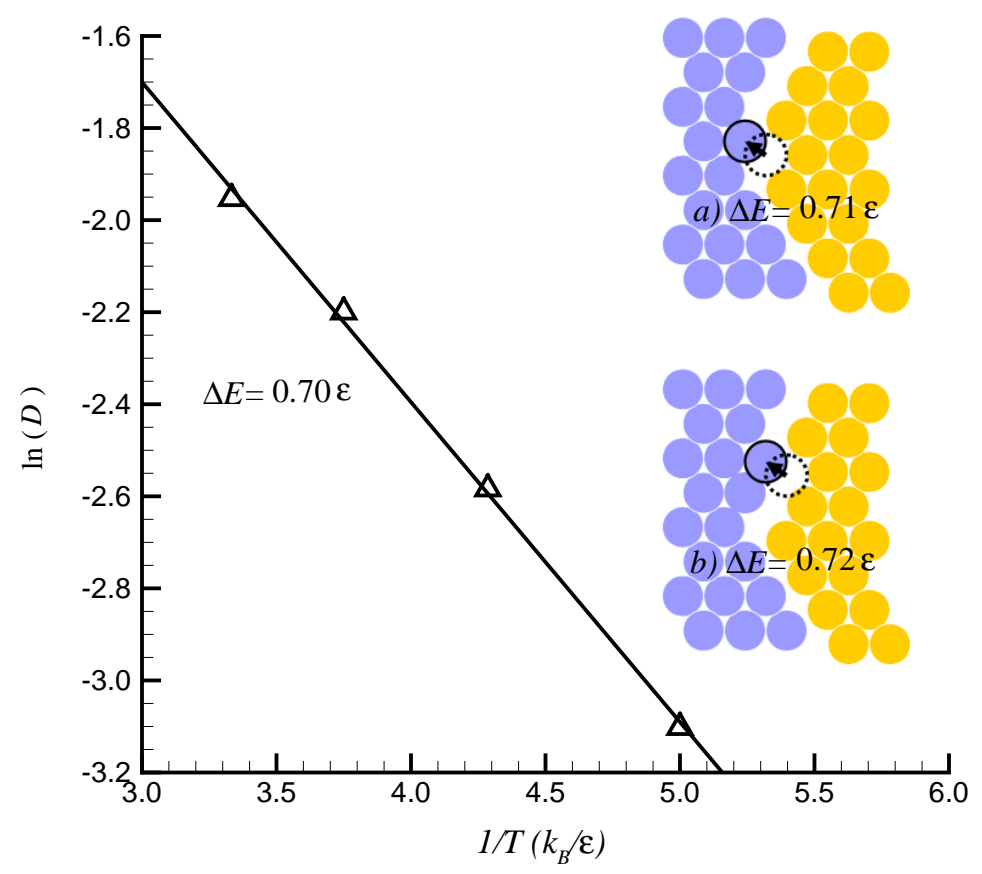

Figure 4.25: The dependence of the log of the grain boundary diffusion coefficient, $D$, on temperature, calculated using MD. The slope of the plot gives an energy barrier $\Delta E=0.70 \epsilon$. As expected this is very close to the barrier for the "kink flip" move (shown in the inset) calculated using the NEB method, which limits the diffusion of the boundaries. 


\subsection{Summary}

In this chapter we have used MD simulations with the Lennard-Jones potential to model dynamic stacking fault grain boundaries in an epitaxial layer on a (111) surface. These simulations reproduce experimentally observed features where stacking fault grain boundaries are composed of a mix of A-gaps and B-gaps. The grain boundaries continually alter their shape and position. The grain boundaries migrate over the surface following a temperature dependent random walk. Over long time periods a slight drift in grain boundary position is observed, in addition to the random diffusion. A pair of grain boundaries can meet and annihilate each other, eliminating the stacking fault. The key atomic process for grain boundary migration is shown to be the "kink flip" move, as proposed by Busse [146]. 


\section{Chapter 5}

\section{Hybrid MD KMC Model}




\subsection{Hybrid Methodology}

A hybrid MD KMC modelling methodology has been developed to deal with the long time scales involved in modelling epitaxial growth, while maintaining the fidelity provided by MD in key domains. In this work the hybrid methodology has been applied to follow the propagation of a stacking fault grain boundary between FCC and HCP islands nucleating on a FCC (111) surface.

This hybrid methodology combines Molecular Dynamics and Kinetic Monte Carlo methodologies within a single concurrent atomistic simulation. Different domains within this hybrid simulation are modelled with different methodologies (MD or $\mathrm{KMC}$ ) and as the simulation progresses the methodology applied to a given domain is changed (perhaps several times) as necessary, so that the stacking fault grain boundary is always modelled with MD, as it moves about the surface.

A great advantage of this hybrid methodology is that when compared to an equivalent full MD simulation, the computer time for the hybrid simulation is reduced in proportion to $\frac{M}{N}$, where $M$ is the number of atoms in MD domains and $N$ is the total atoms. The reduction in computational cost of the hybrid methodology comes largely from avoiding the computation of forces between pairs of KMC atoms. If the system is described by a short-range potential with a cut-off, then the MD force calculation employing Verlet lists (Chapter 3) would ordinarily scale as $O(N)$, where $N$ is the total number of atoms. In this case, a domain decomposition with distinct KMC domains that are significantly larger than the force cut-off will maximise the number of pairs of $\mathrm{KMC}$ atoms that can be ignored in the force calculation.

\subsection{Features of the Hybrid Method}

\subsubsection{Methodology Domains}

In this work our goal is to follow the propagation of grain boundaries between FCC and HCP islands nucleating on an FCC (111) surface. 
The KMC methodology is only able to simulate a set of predetermined events, whereas MD simulates all changes resulting from the forces between the atoms. Therefore our hybrid approach applies MD to those domains with the most complexity, i.e. in proximity to the grain boundaries. The remaining atoms are contained in KMC domains. The system is adaptively partitioned so that the grain boundaries are always contained in MD domains. This split of methodologies into distinct domains reduces the computational cost compared to applying MD to all the domains. The MD domains contain the grain boundaries so that accuracy is retained where it is important to do so.

\subsubsection{Domain Interface}

However, there are several artefacts that may potentially arise at the interface between the MD and KMC domains. The first involves the reflection of waves from the rigid atoms in the KMC lattice. This problem also arises in hybrid MDcontinuum methods, and several techniques have now been proposed to deal with this issue.

To and Li [147] have developed an approach called the Perfectly Matched Layer (PML). The PML is sited between regions modelled with distinct methodologies, where it damps out spurious reflections of waves. In the PML, a damping term proportional to velocity is applied to atom motion. In addition, the spacing between the atoms is adjusted, thereby changing the stiffness of the system. E and Huang [148] approach the problem of minimising the reflection of waves from an atomistic-continuum interface by effectively applying additional forces at the interface. Cai et al. [149] develop time dependent boundary conditions in order to reduce the reflections of elastic waves.

We note that the rigidity of the KMC domain will also bias the effective hopping rates of $\mathrm{MD}$ atoms near the interface. As the primary object of interest in this study is the migration of grain boundaries, we have dealt with these artefacts by i) applying a Langevin thermostat [96] to the atoms in the MD domains to damp the reflection of waves (in view of the use of such damping in the PML scheme) and 
to keep the MD atoms in thermal equilibrium, and ii) increasing the widths of the MD domains until we observed the convergence of the grain boundary diffusion coefficient.

\subsection{Initial Configuration}

\subsubsection{Arrangement of Atoms}

We have simulated the behaviour of a stacking fault grain boundary in the top atomic layer of a (111)-terminated FCC slab of atoms. As discussed in Chapter 4, parts of the top atomic layer are in HCP stacking, producing a stacking fault grain boundary where they meet the FCC stacking atoms in the top layer. The stacking fault grain boundary is initialised as a missing row of atoms across the narrow direction (width) of the slab. The details of the slab of atoms are given in Chapter 4.

\subsubsection{Domain Geometry}

The initial configuration of a slab with an FCC stacking island in its centre and HCP islands on its ends is shown in Figure 5.1 (Top). A close-up of one of the grain boundaries on this slab, in its initial configuration, is shown in Figure 5.1 (Bottom). 


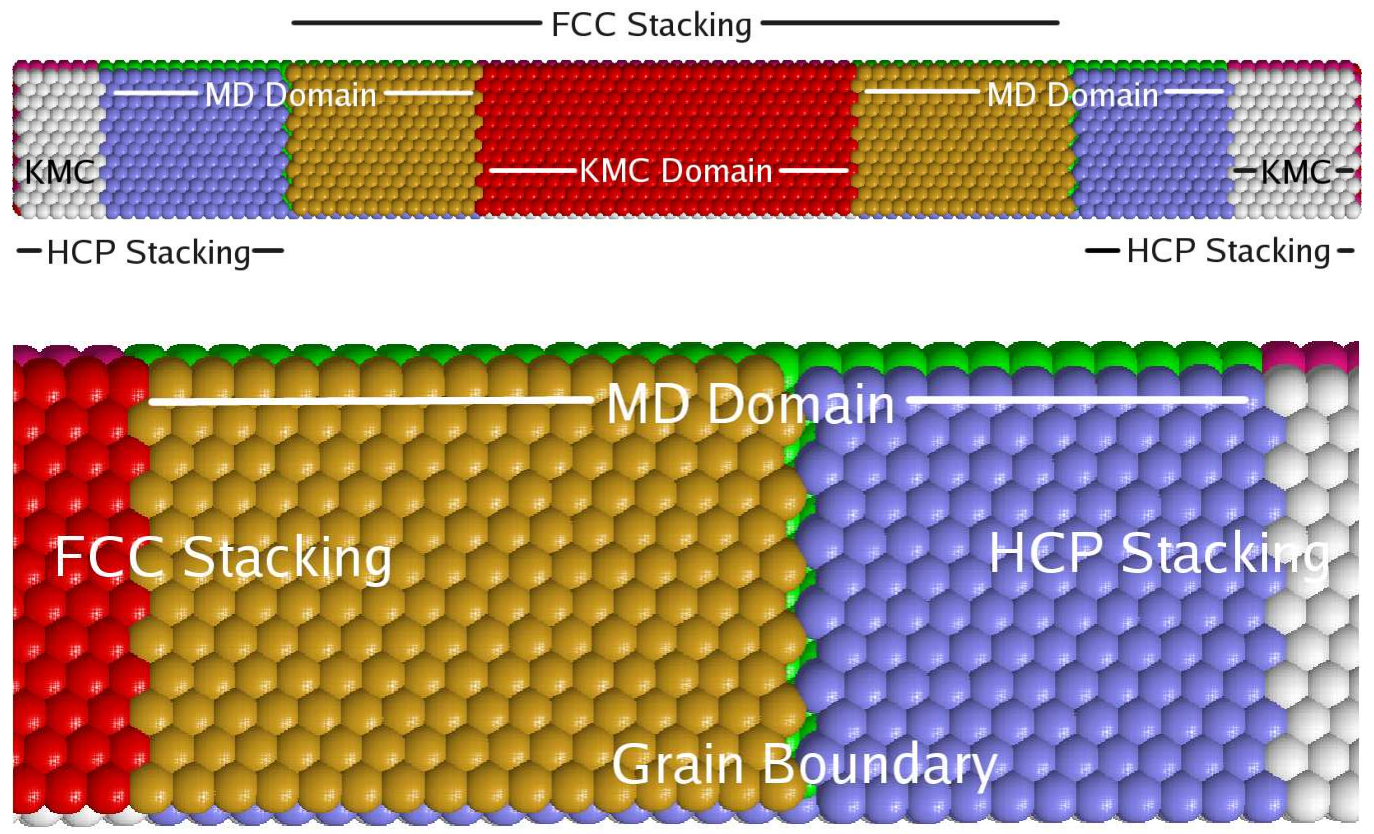

Figure 5.1: (Top) View from above looking down on the initial configuration of grain boundaries on a slab. The blue and gold atoms in the MD domains are on HCP and FCC sites respectively, while the grey and red KMC atoms are located on $\mathrm{HCP}$ and FCC sites respectively. The grain boundaries lie in the MD domains at the juncture of the blue and gold MD atoms. (Bottom) Close up of a grain boundary in its initial configuration. Because the blue and gold MD atoms are on different sites, their respective islands cannot interlock at the grain boundary. The atomic scale "gap" at the grain boundary is sufficient to allow atoms to move back and forth at random across the grain boundary from one stacking island to the other. Over time this atomic motion changes the shape of the grain boundary and also alters its location along the slab, as one island type grows at the expense of the other.

The slab is of the same design as the slab used in the MD simulations in Chapter 4. The pair of grain boundaries are contained in separate MD domains, with $\mathrm{KMC}$ domains encompassing the rest of the atoms in the slab. Because of the periodic boundary conditions, the domains at either end of the slab are effectively 
one. When the configuration of the slab is written out, the atoms are colour coded to identify the MD and KMC domains on the slab. This colour coding also shows which stacking, FCC or HCP, applies to a particular domain. Table 5.1 gives the correspondence between domains, stacking and colours. The colour coding enables the location and extent of the domains and stacking islands to be followed visually during a simulation.

Table 5.1: Atom Colour Code

\begin{tabular}{ccc} 
Domain & Stacking & Colour \\
\hline KMC & HCP & Grey \\
KMC & FCC & Red
\end{tabular}

MD HCP Blue

MD FCC Gold

Initially there are four domains, two MD domains (containing their respective grain boundaries) and two longer KMC domains. One KMC domain is between the two MD domains and the other "split" around the ends of the slab. As the simulation progresses, migration of the grain boundaries towards each other may result in the (temporary or permanent) coalescence of the two MD domains into one, eliminating the central KMC domain as well, as occurred in the MD simulations.

The domains are rectangular in shape covering the width and depth of the slab. The interface between adjacent MD and KMC domains is a flat surface parallel to the narrow end of the slab, covering the width and depth of the slab. At this interface, the MD atoms "feel" a wall of immobile KMC atoms.

The MD domains are each of length $\mathrm{L}$ with the KMC domains making up the remaining length of the slab. 


\subsubsection{Domain Length}

The length of the MD domain, L, is a key element in the hybrid methodology developed here. The MD domain needs to be of sufficient length that the dynamics of the grain boundary contained within it is unaffected by the presence of the blocks of immobile atoms comprising the adjacent KMC domains. At the same time the number of MD atoms, and hence L, should be kept small to minimise the computational cost of the hybrid model, which is dominated by the computational cost of MD. Figure 5.2 shows the results of several simulations using the domain decomposition algorithm without $\mathrm{KMC}$ events. Atoms in the $\mathrm{KMC}$ domains remain fixed on the lattice unless the grain boundary migrates, in which case the adaptive domain decomposition converts these domains to MD domains to keep the MD domain centred on the grain boundary.

Using this approach we have examined the dependence of grain boundary migration on the length L of the MD partition. In Figure 5.3, we plot the mean square displacement in a time interval versus this time interval for a variety of MD domain lengths, including a full MD simulation without partitioning. Once again the linear growth with time indicating a diffusive random walk is seen. Note the convergence of $\left\langle\Delta x(\Delta t)^{2}\right\rangle$ to that of the full MD result as the length of the MD domains increases with good agreement when $\mathrm{L}=30 \sigma$.

The default length $\mathrm{L}$ of an MD domain is thus set to be $30 \sigma$. As a simulation progresses, a grain boundary can become more convoluted and take up a greater length of the slab, i.e. the width of the grain boundary may grow as discussed in Chapter 4. A sufficiently wide grain boundary may spread, requiring an increase in the size of the MD domain. The algorithm used will increase the size of the MD domain where necessary during the course of the simulation, as discussed further in section 5.4.3. The configuration of a grain boundary can fluctuate considerably during a simulation. Thus the length of its encompassing MD domain can also vary, subject to the minimum length of $30 \sigma$. 

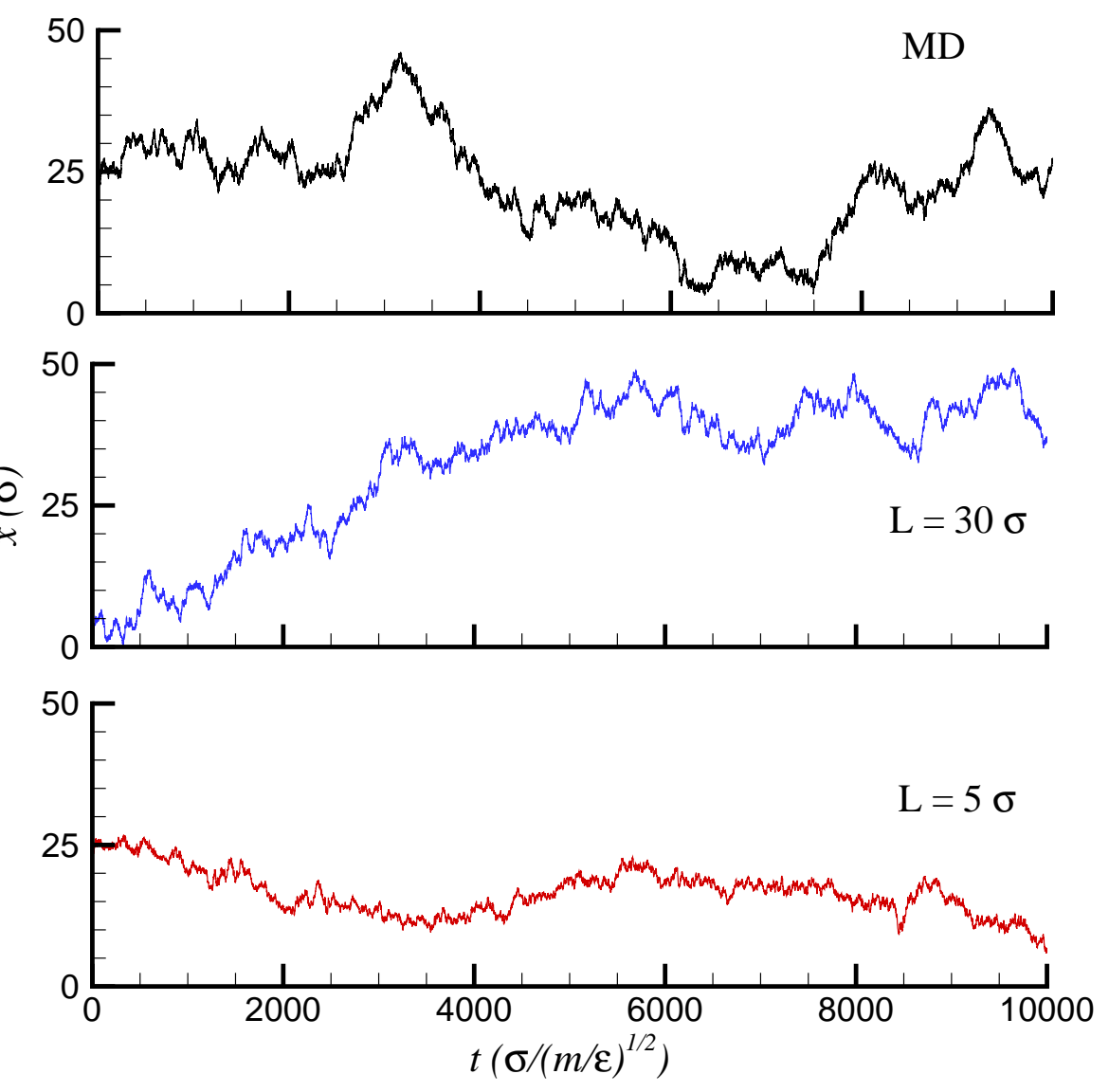

Figure 5.2: Evolution of the grain boundary position (being the average position of the boundary across the width of the slab) during typical simulations using the MD method, the hybrid method with $\mathrm{L}=5 \sigma$ and the hybrid method with $\mathrm{L}=30 \sigma$. The mobility of the boundary in the $\mathrm{L}=5 \sigma$ MD domain is clearly suppressed. 


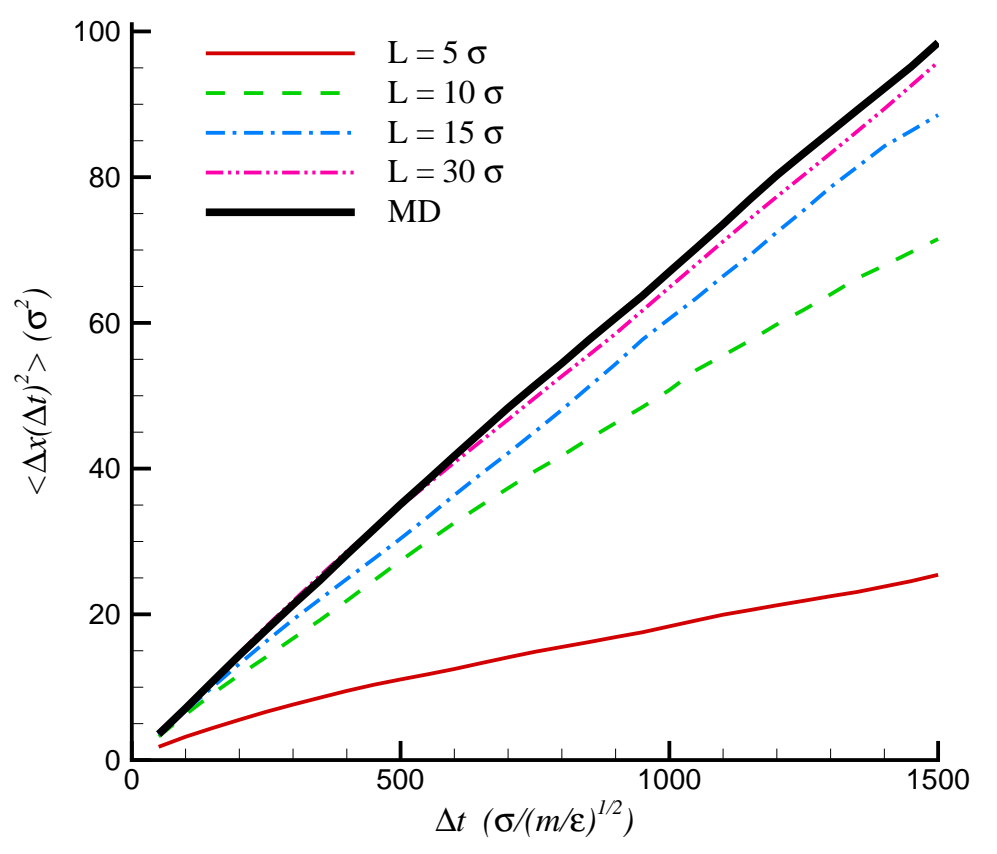

Figure 5.3: The mean square displacement $\left\langle\Delta x(\Delta t)^{2}\right\rangle$ versus $\Delta t$ for the MD simulation and for the hybrid method for various MD domain sizes. The slight sublinearity observed in the MD simulation graph is just due to limited statistics - as the results of more runs are included in the average the graph becomes linear for longer durations. 


\subsubsection{KMC Lattice}

Because of the existence of both FCC and HCP sites on the (111) surface of the slab, the usual triangular lattice is not sufficient for the KMC lattice in this simulation. The grain boundaries separate islands of atoms on FCC sites from islands on HCP sites, so the KMC lattice must be constructed to allow both FCC and HCP sites to be identified. A "refined lattice" with a finer triangulation mesh than the (111) nearest neighbour triangulation was used. The KMC atoms occupy positions on this refined triangular lattice given by $\mathbf{r}=n_{a} \mathbf{a}+n_{b} \mathbf{b}+n_{c} \sqrt{2} \mathbf{c}$, where $|\mathbf{a}|=|\mathbf{b}|=|\mathbf{c}|$ and the $\mathbf{a}$ and $\mathbf{b}$ axis lie in the (111) plane, the plane of the slab. They cross at a sixty degree angle and the $\mathrm{c}$ axis is normal to the surface of the slab. In this coordinate system an atom at position $\mathbf{r}$ in a close-packed facet will have three nearest neighbours at either $\{\mathbf{r}+\mathbf{a}+\mathbf{b}, \mathbf{r}+\mathbf{a}-2 \mathbf{b}, \mathbf{r}-\mathbf{a}-\mathbf{b}\}$ or $\{\mathbf{r}+2 \mathbf{a}-\mathbf{b}, \mathbf{r}-2 \mathbf{a}-\mathbf{b}, \mathbf{r}-\mathbf{a}+2 \mathbf{b}\}$, as shown in Figure 5.4. Note that the nearest sites will be unoccupied in a closest packed plane, but in the course of a simulation any of the sites in the lattice could become occupied at some point. The nearest neighbour distance for an FCC crystal in this coordinate system is $\sqrt{3}|\mathbf{a}|$.

$\mathrm{KMC}$ atoms can only move to or be deposited on sites which are unoccupied, have unoccupied neighbouring sites and are supported by a triad of nearest neighbor atoms in the layer below. Movements of KMC atoms in the plane are restricted to a subset of the six neighbouring lattice sites $\{\mathbf{r} \pm \mathbf{a}, \mathbf{r} \pm \mathbf{b}, \mathbf{r} \pm(\mathbf{a}-\mathbf{b})\}$, to prevent atoms being sited within the nearest neighbour distance of each other. 


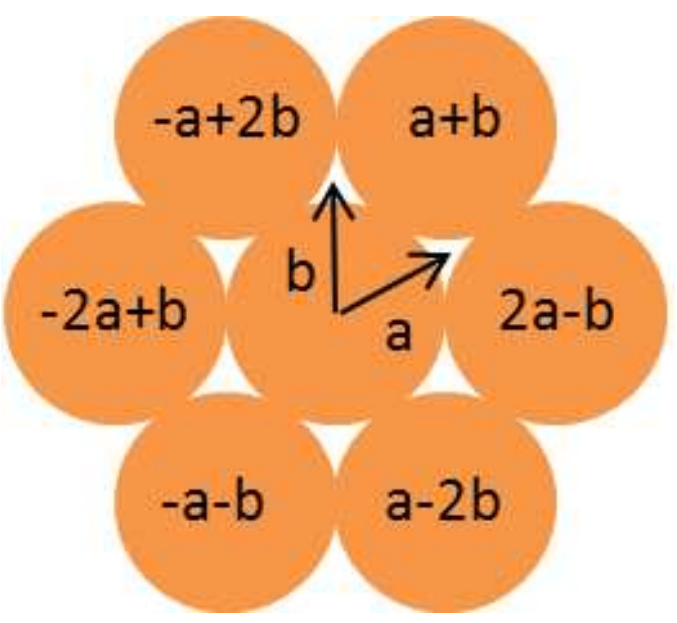

Figure 5.4: The in the (111) plane refined lattice vectors $a$ and $b$ are shown with respect to the site of the central atom. The six in plane nearest neighbour atoms are labelled with their relative positions in terms of $a$ and $b$.

\subsection{Hybrid Algorithm - Domain Adaptivity}

\subsubsection{Algorithm}

The Algorithm for the hybrid Methodology is composed of the following steps.

- Initialisation

- Initial Domain Identification

- Dynamic Steps: repeat the following steps for the duration of the simulation

- KMC Step

- MD Step

- Domain Identification

- Data Collection

The initialisation step reads in the coordinates of the atoms in the slab in its initial configuration. Atoms are then given velocities consistent with the temperature 
set for the simulation. In the domain identification step the slab is divided up into MD and KMC domains. The MD domains are set so that they contain the grain boundaries. Grain boundaries are recognised when rows of atoms across the width of the slab contain a mix of FCC sited and HCP sited atoms. The dynamic phase now follows, where for the duration of the simulation there is a sequence of a KMC step applied to the KMC domains, followed by an MD step. Each KMC step carries out a KMC event on a KMC domain and increases the amount of time simulated. The subsequent MD step performs an MD simulation for a period equal to the time increase from the previous KMC step. At the start of the MD step, any atoms that were in a KMC domain in the previous iteration but are now encompassed in an MD domain are assigned velocities consistent with the temperature, i.e. their kinetic energies are determined from the temperature of the simulation using the equipartition theorem. Before going onto the next KMC step the division of the slab into MD and KMC domains is checked and adjusted if necessary. When a grain boundary changes its extension along the slab or its average position, the encompassing MD domain may need to have its size or location adjusted and the adjacent KMC domains are also adjusted accordingly. Periodically, data on grain boundary positions and the atomic configuration of the slab are written to files.

\subsubsection{Initialisation}

In the initialisation stage of a simulation, a status list classifying each atom as mobile or fixed is constructed. This list is subsequently used to determine which simulation methodology will apply to a given atom at a particular time in the simulation. Those atoms classified as mobile are subject to the MD methodology, while those classified as fixed are treated by KMC. At first all the atoms are classified as being mobile. A list of the Langevin damping parameter $\gamma$ (see Chapter 3 ) to be applied to each atom is also constructed. The standard value is $\gamma=1.0$ in these simulations. All atoms in the initial configuration are given random initial velocities such that their initial kinetic energies correspond to the kinetic tem- 
perature chosen for the simulation. The standard temperature for the simulations is a reduced temperature of $T=0.35$. The average velocity is then subtracted (component-wise) from each atom's velocity, to remove any linear momentum of the slab as a whole. This ensures that velocity is measured in the center of mass frame and the thermostat maintains the temperature set. For each simulation a different random seed is used to generate the initial velocities, thus making each simulation a distinct realisation of the evolution of the grain boundaries.

\subsubsection{Initial Domain Identification}

For the purposes of determining the location of the MD and KMC domains, the length of the slab is divided into bins, each covering the full width and depth of the slab but of length $10 \sigma$. These bins are effectively a series of stripes traversing the slab along its length. Each atom in the slab is then allocated into the bin that encompasses it. For each bin the siting (FCC or HCP) of its top layer atoms is calculated, based on the lattice site of each atom (see section 5.3.4). Only those bins with a mixture of HCP and FCC sited atoms contain a grain boundary. The bins that contain a grain boundary, plus the adjacent bin on either side (buffer bins), together comprise an MD domain. Initially a grain boundary is fully encompassed by a single bin. In between updates of the location and extent of the MD domain, the buffer bins of dynamic atoms on either side provide some space for the grain boundary to migrate or to take up a more convoluted shape, without being impeded by fixed KMC atoms. The atoms in the remaining bins have their status set to fixed and comprise the KMC domains. The atoms in the bottom layer of the slab remain static throughout the simulation.

\subsubsection{Dynamic Steps}

Once initialisation is complete and the location and extent of the initial MD and KMC domains have been determined, a series of dynamic steps is carried out. Each dynamic step comprises a KMC step followed by an MD step for the same 
duration of simulation time. These steps update the atom configuration of the slab. Before the next dynamic step, the MD and KMC domains are reviewed and adjusted if there has been any significant change in the location or extent of the grain boundaries.

\subsubsection{KMC Step}

For atoms in the KMC domains the energy barriers to hop to a neighbouring site are determined from the number of nearest neighbours, as discussed in Chapter 3. The KMC routine attempts to choose a KMC atom from one of the KMC domains to hop from its current lattice site to an empty adjacent site. The fixed atoms which are all in KMC domains are located exactly on lattice sites. However, the dynamic atoms in the MD domains are not in general exactly on lattice sites, as they continually vibrate about their equilibrium positions (the lattice sites). Consequently, for KMC atoms at the interface between a KMC domain and an MD domain, both the number of their nearest neighbours and the number and location of adjacent vacant sites is uncertain. To resolve this issue, the KMC routine notionally allocates each MD atom to its nearest vacant lattice site, which in most instances is where it would reside if it had been simulated as a KMC atom from the start.

A table of nearest neighbours and adjacent vacant sites for each $\mathrm{KMC}$ atom is then built. This gives the list of possible hops for the current atomic configuration. One hop is picked at random (but in proportion to the hop rates of the currently possible hops) from this list. The chosen atom is then moved to its new location.

This hop advances the simulation time by an increment $t_{n}$ [138], which depends on the hopping rate for the chosen hop. If the hop has a low energy barrier (i.e. the chosen atom has few neighbours) compared to the energy available ( $k_{B} T$ ), then such a hop would be expected to occur frequently and occur in a short time, with a much longer time expected when the energy barrier is larger. For example, with an attempt frequency of $10^{13}$, temperature of 0.35 and energy barrier of 0.3 the expected time for a hop is on the order of $10^{-13}$, while for a larger energy barrier of 3.3 the expected time is on the order of $10^{-7}$. Thus the time increment 
$t_{n}$ is essentially this expected occurrence time. The rate for a KMC hop depends on the location of neighbouring MD (as notionally allocated to lattice sites) and KMC atoms. The more neighbours, the more difficult it is for an atom to hop away from its current site and the lower its hop rate. If the advance in time $t_{n}$ is less than $1 t$, the KMC step is repeated (as often as necessary) until the aggregate time advance $\sum t_{n}$ exceeds $1 t$. This ensures a minimum time period of $1 t$ for the subsequent MD step to cover. Setting this minimum time avoids short MD steps as these would not produce any significant change in atomic configurations while adding to the computational cost.

\subsubsection{MD Step}

Now there is a period of MD simulation in the same way as in Chapter 4. Any atoms that are new to an MD domain because of resizing of the domains since the last MD step are assigned velocities consistent with the simulation temperature. To ensure that the MD and KMC domains interact with contemporaneous versions of each other, the evolution of the MD and KMC domains is synchronised as much as possible. Thus the MD step, in which the positions and velocities of all the dynamic MD atoms are updated (stepped forward in time) by an MD time step $\delta t=0.01$, is run $\frac{\sum t_{n}}{\delta t}$ times to move forward the MD domains by the same amount of simulation time as the KMC domains.

\subsubsection{Inter-Domain Transfers}

As a result of the hop(s) during the $\mathrm{KMC}$ step, an atom that was in a $\mathrm{KMC}$ domain may now be located in a MD domain. In that case its status is changed to a mobile $\mathrm{MD}$ atom and it is given a random velocity consistent with the temperature $T$ as in the initialisation stage. Also, as a result of (subsequent) MD steps, an MD atom may diffuse into a KMC domain; its status is then immediately changed to a fixed $\mathrm{KMC}$ atom and it is placed on the nearest vacant lattice site. 


\subsubsection{Domain Location Update}

At the end of each dynamic step the atoms are repartitioned into KMC and MD categories, using the same methodology employed in the initial decomposition. The time interval between these updates to the domain locations, is usually of the order of $1 t$ or $100 \delta t$. From the square root of the slope of the MSD graph for full MD simulation (Figure 5.3), we can estimate the typical displacement of a grain boundary as $0.26 \sigma$ per $1 t$. In the repartitioning, those atoms with $y$ coordinate (long axis of the slab) within $15 \sigma$ of the corresponding $y$ position of a grain boundary become MD atoms while the rest are classified as KMC atoms. In this way the MD domains remain centred around the grain boundaries even as the grain boundaries change their shape and migrate over the surface of the slab.

\subsubsection{Data Collection}

During each simulation, information on the positions of the grain boundaries are recorded for each integer value of $t$. The positions of the grain boundaries were tracked using the same binning approach employed in identifying the MD domains encompassing the grain boundaries, but using much finer bins of length $1.7 \sigma$. This data was subsequently used to provide time traces of the migration of grain boundaries along the surface of the slab, and to enable calculations of the grain boundary diffusion coefficients to be made. Snapshots of the full atomic configurations were recorded approximately every $100 t$.

\subsubsection{Total Simulation Run-Time}

The required simulated time is a parameter fed into the model at commencement of a simulation run. Typically, simulations were run until the simulation time $t$ reached 10,000, i.e. 1 million MD steps of duration $\delta t$ each. Runs of this length allow for the possibility of grain boundaries diffusing most of the length of the slab, meeting and then coalescing. 


\subsection{Off-Lattice Events}

Any events that are off-lattice (with respect to the lattice used here) will not be captured by KMC. However, the simulations have shown (see section 5.7.1) that atoms on off-lattice sites are important in grain boundary pinning. The use of MD is thus clearly necessary to observe effects such as these which have not already been built into the $\mathrm{KMC}$, and this is a key strength of this hybrid methodology.

\subsection{Effect of Proximity to a KMC Domain}

The atoms in the KMC domain sit rigidly on lattice sites while in the MD domain atoms vibrate about their equilibrium positions. These equilibrium positions are the analogues of the KMC lattice sites. As can be seen in figure 5.5, an extension of the KMC lattice into the MD domain would closely match lattice sites with equilibrium positions in most cases.

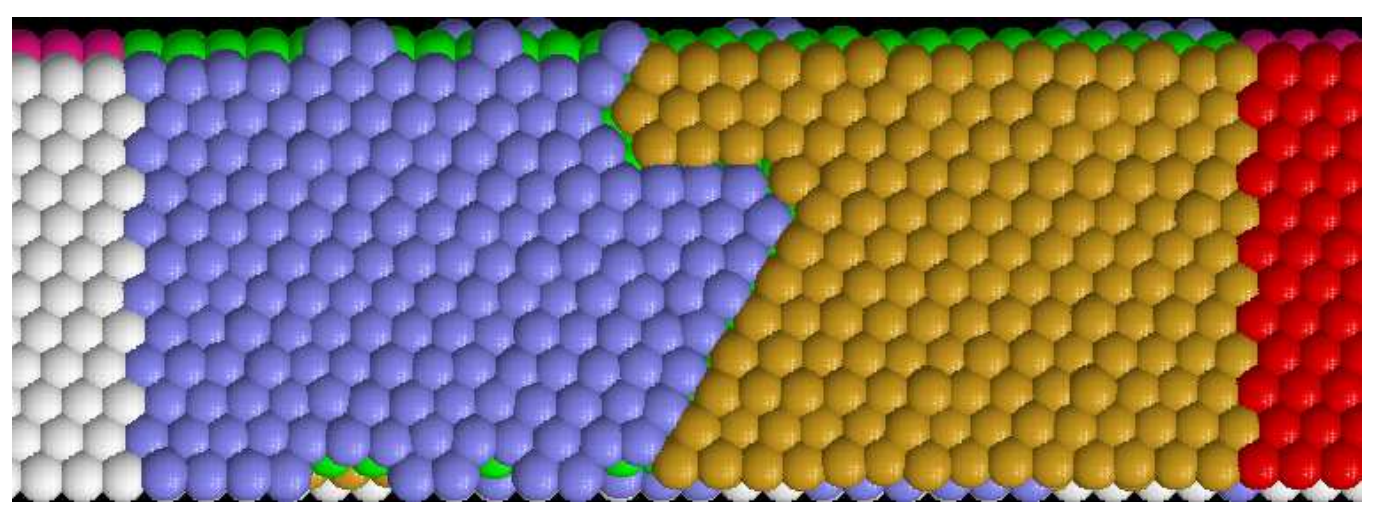

Figure 5.5: Image of a grain boundary in an MD domain which has transformed into the zigzag conformation. The edges of the adjoining KMC domains are visible on the left and right sides of the image. The equilibrium positions of the MD atoms are closely aligned to a continuation of KMC lattice sites

MD atoms further than the force cut-off distance from the KMC domain only 
experience direct forces from other similarly mobile MD atoms. There is the possibility of indirect interactions between MD and KMC atoms further than the cut-off distance apart, due to reflections of elastic waves off rigid $\mathrm{KMC}$ atoms. In contrast, MD atoms within the force cut-off of the KMC domain directly experience forces from the rigid KMC atoms. This alters their motion compared to the rest of the MD atoms. This can be seen from the energy barriers (depths of potential wells) that MD atoms in different locations experience. The energy barriers for key atomic motions (hops between equilibrium positions) when the $\mathrm{MD}$ atoms were in close proximity to the KMC domain were calculated using the NEB method. Figure 5.6 shows the results of these calculations. These calculations were carried out using an MD domain of length $30 \sigma$, with the grain boundary established at a number of different distances from the KMC domain in each case. When atoms in the grain boundary are within approximately $5 \sigma$ of the KMC domain, the energy barriers for "corner crossing" and "kink flip" hops are affected. The "kink flip" hop energy barrier increases, making "kink flip" hops less frequent. Conversely, the "corner crossing" hops are slightly more frequent. Overall the net effect is a small reduction in grain boundary mobility when they are close to KMC domains.

This effect of the proximity of rigid KMC atoms on the motion of MD atoms also indicates a minimum size for L, the length of the MD domain. Figure 5.7 shows the change in "kink flip" and "corner crossing" energy barriers for various values of L. These show larger changes than in Figure 5.6, because MD atoms are now in close proximity to two KMC domains. The distance of MD atoms in the grain boundary from a wall of $\mathrm{KMC}$ atoms needs to be more than approximately $8 \sigma$ to avoid any effects. Hence on these grounds $\mathrm{L}$ should be at least $16 \sigma$.

These effects of the proximity of KMC domains (and their walls of rigid KMC atoms) on MD atom energy barriers, and hence motion, are small and localised to a range of approximately $8 \sigma$. Consequently they are not sufficient to fully explain the reduction in grain boundary mobility observed when the MD domain length $\mathrm{L}$ is less than some $30 \sigma$. A further small part in the explanation may be some reduction in MD atom vibration rates due to the proximity of KMC domains. A 
greater part of the explanation, however, is thought to be due to elastic interactions between MD atoms and the walls of rigid KMC atoms. Any elastic waves propagating in the MD domain that meet the KMC walls will be completely reflected, without diminution, back into the MD domain. In contrast, such waves would continue to propagate much further, and likely be attenuated, in a simulation that was fully comprised of MD atoms. We think that such nonphysical reflected waves may interfere with the propagation of the grain boundaries. The reflected waves may interact with the normal modes and thereby alter the attempt frequency as determined by the Vineyard formula in Chapter 3. Altering the attempt frequency changes the hop rates of the events that move the grain boundaries. This could be tested by adding wave attenuation regions between the MD and KMC domains and measuring the effect of their presence on grain boundary propagation. In Chapter 6 we discuss ways of reducing such reflections to minimise this artefact and potentially enable a smaller MD domain length $\mathrm{L}$ to be used in the hybrid model.

\subsection{Grain Boundaries and Adatoms}

To demonstrate the full hybrid scheme we have investigated the effect of adatoms on grain boundary mobility. It is important that the rates for events in the KMC model are consistent with corresponding processes in the MD domain. To ensure this we simulated adatom diffusion in a purely MD system at temperatures up to $0.2 \epsilon / k_{B}$. The resulting diffusion coefficient showed the usual Arrhenius dependence on temperature with an energy barrier of $0.29 \epsilon$. This compares well with the barrier height estimated using the NEB method of $0.31 \epsilon$. In addition, we were able to compute the prefactor for the KMC rate using the MD simulations which has a value of $0.38\left(\mathrm{~m}^{2} / \epsilon\right)^{1 / 2}$. As the main role of $\mathrm{KMC}$ is to model adatom diffusion, rates for other processes were computed using this prefactor, and the energy barrier calculated using the NEB method combined with a bond counting scheme. 


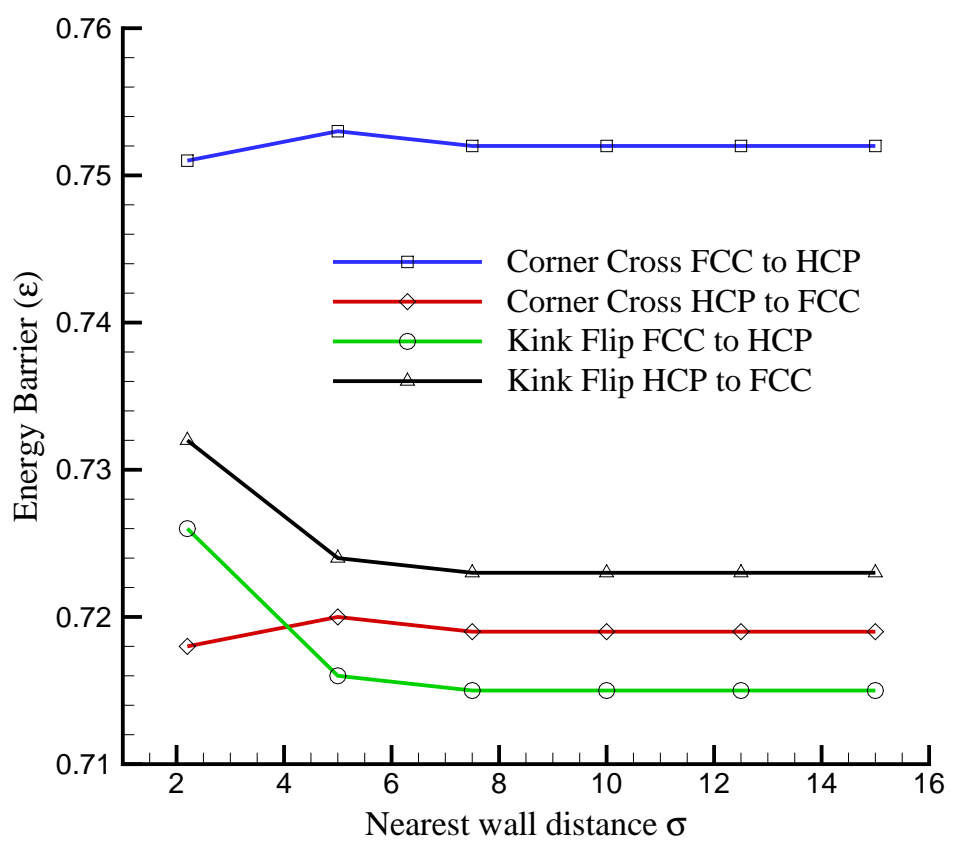

Figure 5.6: This figure shows how the energy barriers for the "kink flip" and "corner crossing" hops are effected by proximity to the KMC domain and its rigid atoms. Once the grain boundary is more than approximately $5 \sigma$ from the KMC domain there is essentially no effect on these energy barriers 


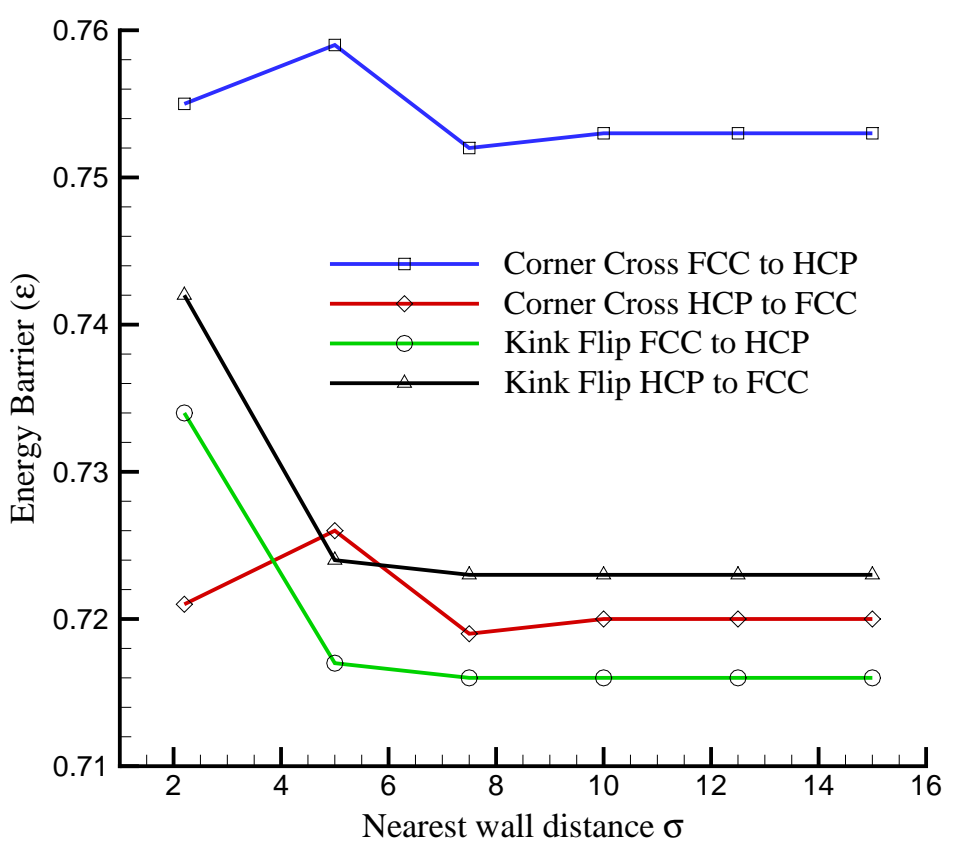

Figure 5.7: The energy barriers for the "kink flip" and "corner crossing" hops in this figure were carried out using grain boundarys centrally placed in MD domains of various lengths $\mathrm{L}$, from $3 \sigma$ to $30 \sigma$. In each case the "kink flip" and "corner crossing" energy barriers were determined using the NEB method. The grain boundary needs to be more than approximately $8 \sigma$ away from any KMC domain to avoid artefacts. 


\subsubsection{Pinning by Adatoms}

Using the set-up discussed above we simulated boundary movement using the synchronised hybrid method for a variety of adatom coverages. In these cases the adatoms were initially randomly distributed over the top surface of the slab. They may diffuse across the boundaries between MD and KMC domains, in which case they are treated in the same way as inter-domain transfers. These simulations used a reduced temperature of $T=0.35$ and a Langevin damping parameter $\gamma=1$. They were run for 10,000 reduced time units. Figure 5.10 compares the displacement in time of a boundary at $2.5 \%$ adatom coverage with a boundary at $30 \%$ adatom coverage. Clearly, at $30 \%$ coverage the grain boundary becomes pinned for long periods of time by adatoms or adatom islands, as indicated by the steps in the $30 \%$ trace. At intermediate levels of coverage, intermittent pinning of grain boundaries can be observed. In Figure 5.8 the time trace shows temporary pinning of a grain boundary at $10 \%$ adatom coverage, by interaction with an adatom island. This pinning effect is also summarised in Figure 5.11, which shows $\left\langle\Delta x(\Delta t)^{2}\right\rangle$ vs $\Delta t$ for a variety of adatom coverages. Also shown in the inset is one of the principal pinning mechanisms identified, where a kink site has become decorated by a diatomic island. Note that the pinning atom is in an off-lattice position, sitting in a fourfold hollow above the A-gap. This effect has recently been observed experimentally in homoepitaxial growth on Ir (111) [146]. Other possible mechanisms for pinning include: a line of adatoms decorating an A-gap away from a kink site, adatoms inserting into the space at the intersection of an A-gap and a B-gap, and an adatom inserting into the top layer of atoms either in a B-gap portion of a grain boundary or in a vacancy site close to the grain boundary. Highly convoluted grain boundaries may offer more sites for adatom insertion and subsequent boundary motion pinning.

\subsubsection{Trapping by Adislands}

Trapping or constraints on grain boundary motion are also caused by larger adislands (aggregations of adatoms). The presence of such adislands makes it difficult 
for grain boundaries to propagate past them. Consequently when such an adisland forms (which can only happen at higher coverage levels), grain boundary motion can be constrained to continually "bounce off" the adisland as shown in Figure 5.8. Similarly a pair of adislands can constrain a grain boundary to oscillate between them. In order for a grain boundary to propagate past an adisland, the atomic stacking in all the surface layer atoms directly beneath the adisland must be changed to the alternate stacking, but the presence of the adisland atoms above hinders this change. The larger the adisland, the more difficult it is for a grain boundary to propagate past the adisland. It would appear that it is only when the part of the grain boundary that meets the edge of the adisland happens to have the appropriate local configuration, e.g. a kink site in the grain boundary meeting the adisland edge, that the boundary can propagate under the adisland. The adislands themselves do not necessarily change their stacking as the result of a grain boundary passing under them.

\subsection{Effect of Varying Langevin Parameter $\gamma$}

The parameter $\gamma$ acts as a drag force opposing the velocity of the MD atoms in a simulation. The temperature is maintained by random velocity "kicks" which, on average, offset the damping due to $\gamma$.

To investigate the effect of $\gamma$ in the hybrid model, sets of hybrid MD KMC runs were carried out with $\gamma=2$ and $\gamma=4$ respectively. In figure 5.12 we show the mean squared displacement of the grain boundary position for these runs, compared to the equivalent results for the standard cases.

As $\gamma$ is increased there is a distinct reduction in the mean squared displacement. That is, by damping the motion of the individual atoms, the mobility of the grain boundary is also reduced.

To gain further insight into how the variation in $\gamma$ altered the mobility of the grain boundary, the effect of varying $\gamma$ on the diffusion of an adatom was studied. For these simulations an adatom was placed on top of a small slab with periodic boundary conditions applied in both in-plane directions. Since there were rela- 


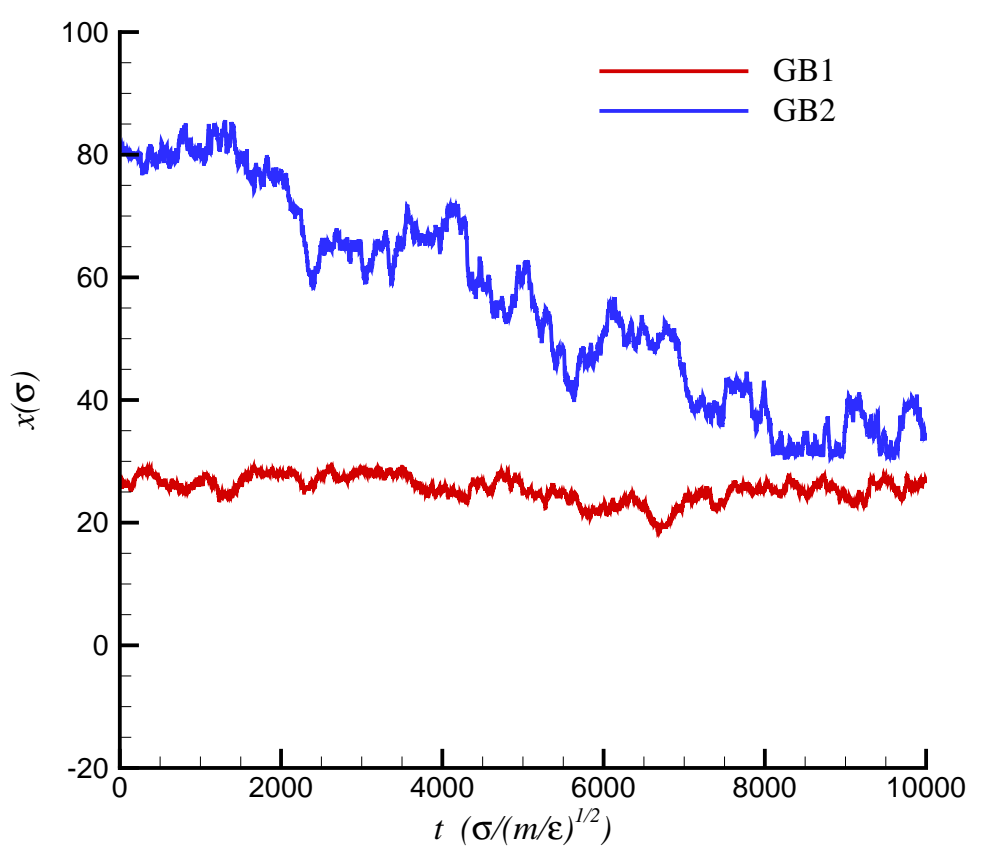

Figure 5.8: Time trace of two grain boundaries in a single simulation with $10 \%$ adatom coverage. Over the time period 8000-9000 the downward motion of the upper grain boundary (GB2) was constrained (as indicated by the flattening of the bottom of the time trace), due to interaction with an adatom island that had nucleated between the two grain boundaries. The motion of GB1 was constrained throughout the simulation. The slab used here was of length $108 \sigma$. 


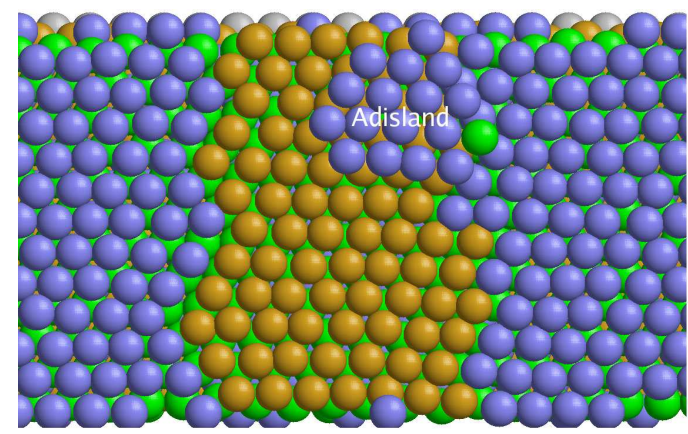

Figure 5.9: This image corresponds to time $8400 t$ in the time trace in Figure 5.8 where the grain boundaries are in close proximity. GB1 is on the left and GB2 is on the right. The adisland that has nucleated on top of the FCC stacking island is preventing GB2 from propagating any further to the left (down the slab in Figure 5.8).

tively few atoms in this full MD simulation, it was feasible to carry out a single run for a relatively long time $300,000 t$, in order to produce sufficient data for statistics. Runs were carried out with a range of values of $\gamma$. In figure 5.13 we see the mean squared displacement of the adatom at different $\gamma$. The same trend of decreasing mobility with increasing $\gamma$ is evident here. The reduction in slope of the mean squared displacement lines, and hence the diffusivity, from the $\gamma=1$ to $\gamma=2$ cases is approximately three times larger than the reduction from the $\gamma=2$ to $\gamma=3$, and $\gamma=3$ to $\gamma=4$ cases. This appears to be distorted somewhat by the upward curve in the $\gamma=1$ case at large time separation. Making an allowance for this, the reduction in slope from $\gamma=1$ to $\gamma=2$ is still approximately twice the reduction of these other two cases.

When the effect of $\gamma$ is only indirect, i.e. $\gamma$ is applied to the atoms in the slab but not the adatom, the pattern of decreasing diffusivity with increasing $\gamma$ is no longer found. In Figure 5.14, it is shown that in this case the mean squared displacement and hence diffusivity are approximately the same for all values of $\gamma$, without any clear trend as $\gamma$ varies. This result makes it seem unlikely that changes in $\gamma$ affect grain boundary mobility through an indirect mechanism. Consequently the effect of variations in $\gamma$ on the grain boundary mobility is expected to be due 

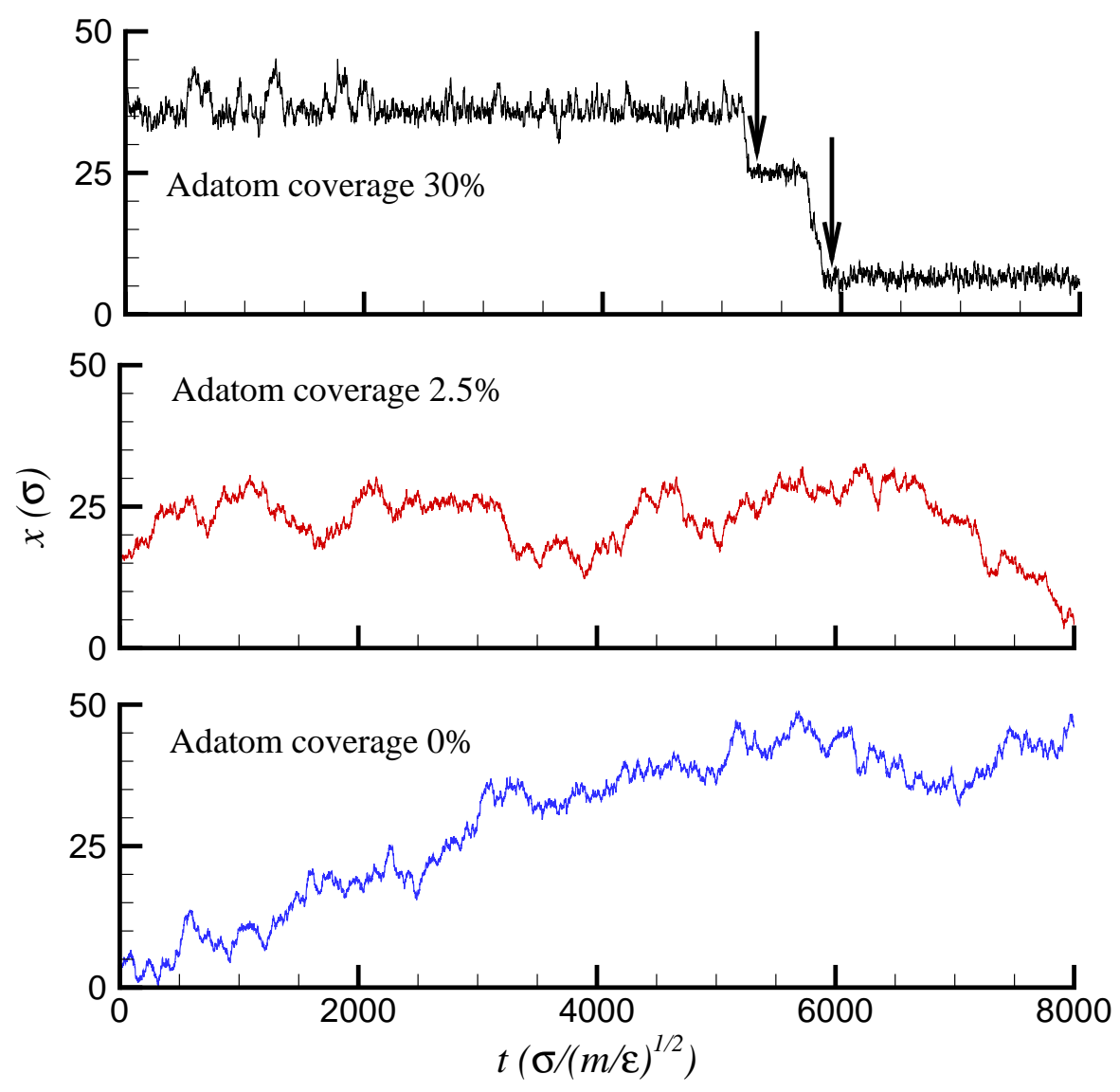

Figure 5.10: Time series showing the movement of grain boundaries at different adatom coverages $(30,2.5$ and $0 \%$ respectively). At $30 \%$ coverage the boundaries are generally pinned by adatoms and adatom islands, although motion can occur when the boundaries pass under islands (two such events are noted by arrows). At $2.5 \%$ the pinning is not observed although mobility is impaired when the boundaries encounter adatoms. 


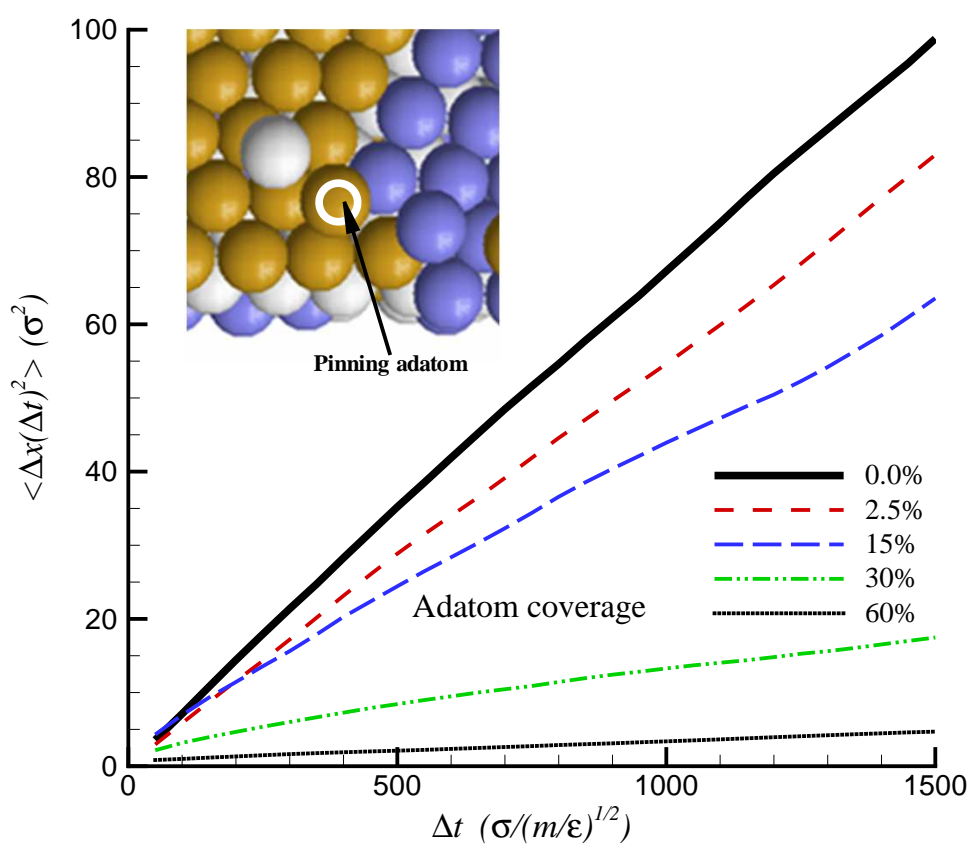

Figure 5.11: The mean square displacement $\left\langle\Delta x(\Delta t)^{2}\right\rangle$ of the boundary versus $\Delta t$ for a range of adatom coverages from $0 \%$ to $60 \%$. The inset shows the main mechanisms for pinning of the boundaries: a kink site decorated by an adatom in an off-lattice position. 
to the direct action of $\gamma$ on those atoms that comprise either side of the grain boundary at any given time.

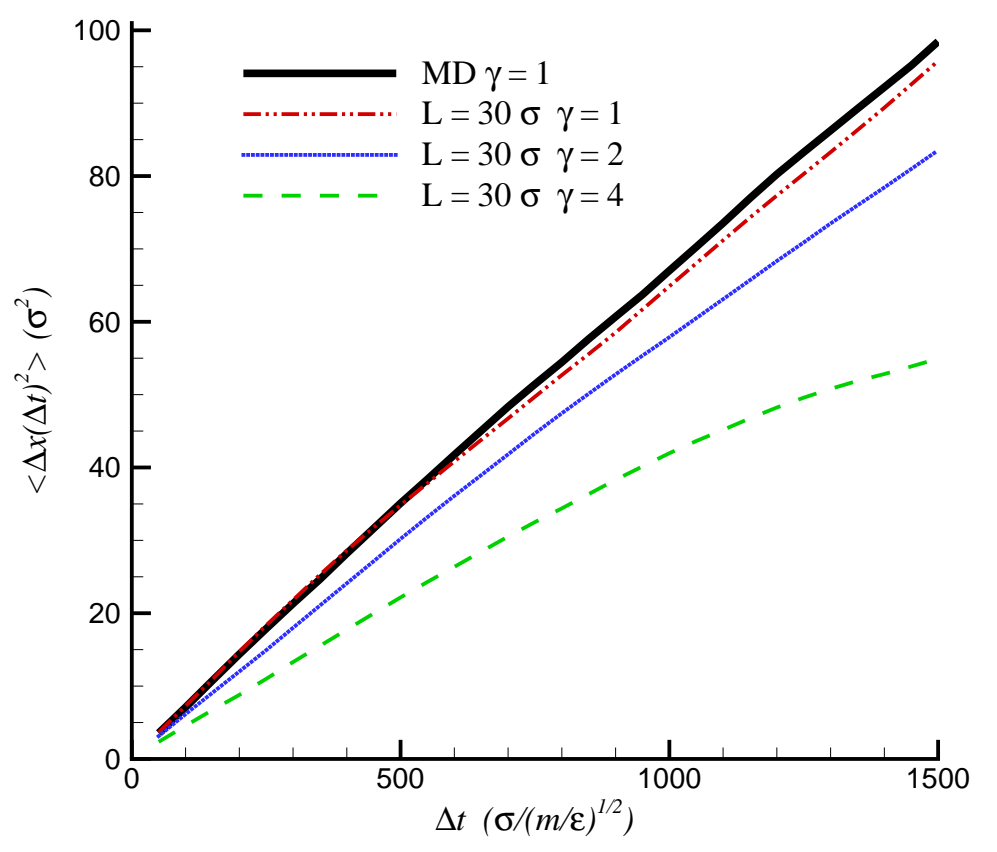

Figure 5.12: In the MD cases and the base MD KMC case, the Langevin parameter is at it standard value $\gamma=1$. From this plot it is clearly seen that the mean squared displacement of grain boundary position reduces as the Langevin damping parameter $\gamma$ increases. In the $\gamma=4$ case, the downward curve of the graph at longer time separations is attributed to the smaller sample size at these longer time separations. The sample size was smaller because of the shorter time series in this case. 


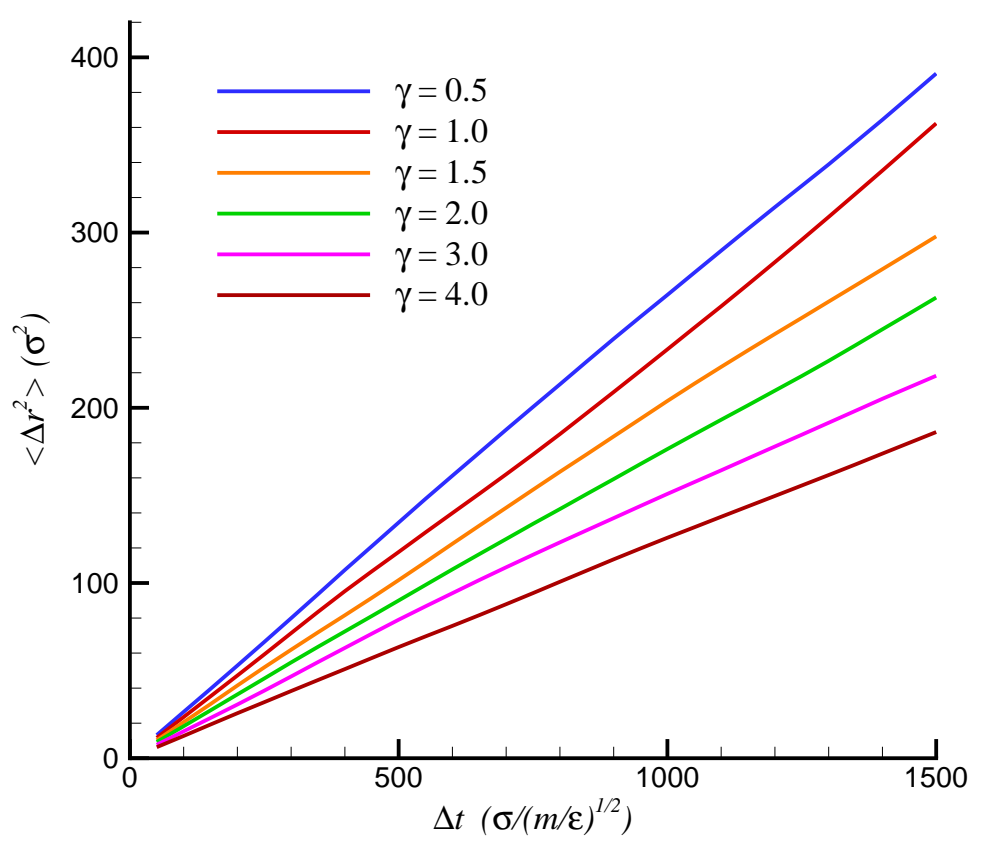

Figure 5.13: This plot shows the mean squared displacement for a single adatom diffusing over a (111) surface. The slope of these lines reduces and hence the adatom's diffusivity declines as the Langevin parameter $\gamma$ increases. In this work the standard value for this parameter is $\gamma=1$. In these adatom diffusion simulations $\gamma$ has been applied to all mobile atoms, including the diffusing adatom. 


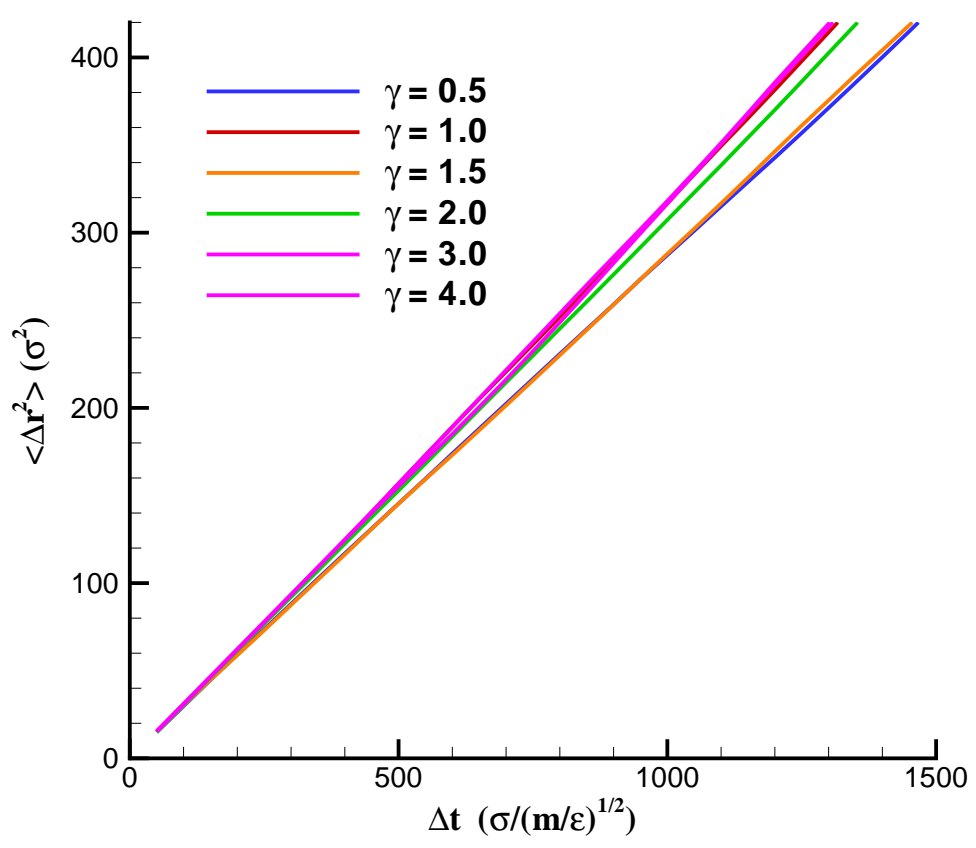

Figure 5.14: The mean squared displacement for a single adatom diffusing over a (111) surface. However in this case, unlike Figure 5.13, $\gamma$ has NOT been applied to the diffusing adatom. Hence when $\gamma$ only indirectly influences the adatom, there is no longer any consistent effect on the adatom's diffusivity.

\subsection{Benefits of the Hybrid Scheme}

\subsubsection{Computational Cost Reduction}

The following table compares the run times for full MD and the hybrid scheme in different sized (total atom number) simulations. Case B uses a slab of atoms more than twice the size of that used in Case A. In all cases, atoms in the bottom layer of the slab are kept fixed to ensure that the slab retains its shape. Hence even in the full MD cases these fixed atoms are not MD atoms (they are not explicitly 
included in the force calculation). All cases ran for a simulated time of $100 t$. The run time is in seconds. In the hybrid scheme, the MD atoms only exist in domains around the grain boundaries, so the number of MD atoms does not scale with the size of the simulation. However, in the hybrid scheme when a grain boundary changes length or curvature, the size of the MD domain encompassing it is adjusted accordingly, altering the numbers of MD and KMC atoms. In this table the average number of MD atoms and KMC atoms during each run is shown.

Table 5.2: Run time comparison.

\begin{tabular}{cccccc} 
Model & Case & Total atoms & MD atoms & KMC/Fixed atoms & Run time (s) \\
\hline Hybrid & A & 5182 & 3235 & 1947 & 2620 \\
MD & A & 5182 & 4318 & 864 & 3356 \\
Hybrid & B & 12350 & 3245 & 9105 & 5808 \\
MD & B & 12350 & 10298 & 2052 & 16599
\end{tabular}

Case A: The reduction in MD atoms in the hybrid run relative to the full MD run is $(4318-3235) / 4318=0.251$ i.e. $25.1 \%$. The run time reduction relative to MD is $(3356-2620) / 3356=0.215$ i.e. $22 \%$. So here a $25.1 \%$ reduction in the number of MD atoms gives a $22 \%$ reduction in run time, relative to the full MD case.

Case B: The reduction in MD atoms in the hybrid run relative to the full MD run is $(10298-3245) / 10298=0.684$ i.e. $68.5 \%$. The run time reduction relative to MD is $(16599-5808) / 16599=0.65$ i.e. $65 \%$. Thus in Case $\mathrm{B}$ a $68.5 \%$ reduction in the number of MD atoms produces a $65 \%$ reduction in run time, with respect to the full MD case.

Overall the decrease in run time equates to the proportionate reduction in MD atoms. This result is consistent with the fact that in MD simulations the force calculation consumes almost all the run time. Consequently, MD run time scales with the number of MD atoms as Verlet lists are used, while in the KMC algorithm used in the hybrid scheme, the run time is essentially independent of the number of atoms and constitutes a negligible part of the computation time. 


\subsubsection{Active Simulation in KMC Domains}

There is as already discussed a saving in run time, since with approximately $32 \%$ of the MD atoms of a full MD run in the runs used to collect the data collected in this Chapter, the hybrid runs took approximately $1 / 3$ of the time of corresponding full MD runs.

Also, it can clearly be seen that the grain boundaries, and hence their encompassing MD atoms, are moving beyond the range of their initial MD domain (length $30 \sigma$ ). This illustrates the adaptive nature of the hybrid scheme.

A most significant feature of the hybrid method is demonstrated in the trace shown at $30 \%$ adatom coverage. In this case the grain boundary is pinned by a structure that has nucleated and grown within the KMC domain before the arrival of the grain boundary. This makes it clear that the KMC domains are not merely passive "walls of atoms" acting only as mechanical boundaries for the active MD domains. In fact the KMC domains are actively simulating atomic interactions and the MD domains and $\mathrm{KMC}$ domains evolve in conjunction, indepenently but consistently. In terms of atomic dynamics the KMC domains act as "coarse grained" versions of the MD domains.

\subsection{Summary}

In this Chapter we have demonstrated the application of our adaptive hybrid method, coupling MD and $\mathrm{KMC}$ in a domain decomposition, to the inhomogeneities that develop in epitaxial growth. This method leads to a speed-up over conventional $\mathrm{MD}$, reducing the execution time by a factor approximately equal to the relative fraction of remaining MD atoms. We have shown that it is possible to reproduce grain boundary mobilities from full MD simulations with the domain decomposition method in the absence of KMC events, when the MD domains are sufficiently large (length $30 \sigma$ ). A lower bound on the MD domain length needed to reproduce grain boundary mobilities is approximately $19 \sigma$. This accommodates the average grain boundary spread/length and maintains sufficient distance from the edges of 
KMC domains. An MD domain length of less than $30 \sigma$ may be effective if propagation of elastic waves within the MD domain can be reduced. This would further reduce the computational cost of the hybrid method compared to full MD for a given simulation. The diffusion of the grain boundaries is shown to reduce as the Langevin damping parameter $\gamma$ is increased. We have also used the method to study the effect of over-layer adatoms on the mobility of the boundaries, demonstrating that boundaries can become trapped by adatom islands. These effects would have been difficult to capture in a conventional KMC simulation. 


\section{Chapter 6}

\section{Enhancing the Hybrid Model}




\subsection{Introduction}

The hybrid MD KMC methodology developed in this thesis employs the two distinct simulation methodologies, Molecular Dynamics and Kinetic Monte Carlo.

These two methodologies are applied in separate regions of the slab of atoms used in a simulation. The atoms in an MD region vibrate about their equilibrium positions and may move to a nearby position. Atoms in a $\mathrm{KMC}$ region are fixed to lattice sites and are only moved one at a time, whenever a KMC event occurs and the configuration of $\mathrm{KMC}$ atoms is changed. MD regions and $\mathrm{KMC}$ regions are positioned alternately along the long axis of the slab.

$\mathrm{MD}$ atoms near an interface between adjacent $\mathrm{MD}$ and $\mathrm{KMC}$ regions cannot impart any of their motion to the rigid KMC atoms. Therefore any waves or patterns of vibrations in the MD region cannot propagate further along the axis of the slab, but are reflected back into the MD region. This is in contrast to the case of using MD exclusively, when such waves would propagate along the slab. Such reflections of waves from an MD KMC interface are artefacts of the hybrid methodology.

These artefacts alter the dynamics of the grain boundaries when compared to a full MD simulation. It is expected that reduction of these wave reflections will enable the hybrid model to operate successfully with smaller MD domains, so further reducing the computational cost of the hybrid model.

In this chapter we investigate the application of a frictional damping force, of the same form as the Langevin parameter $\gamma$, to the reduction of the amplitude of reflected waves. This force is applied in a limited damping region next to the interface between $\mathrm{MD}$ and $\mathrm{KMC}$ regions. A simple 1-D model is developed to estimate the effect of such a damping region within the hybrid MD KMC model.

\subsection{Wave Reflections}

We analyse the theory of wave reflections from a boundary using the simple model of a 1-D linear chain of atoms. Atoms are equally spaced along the 1-D linear 
chain. All the atoms have the same mass. The atoms have nearest neighbour interactions via linear spring forces. Initially all the atoms are at rest in their equilibrium positions. A wave propagating along the chain of atoms is registered as the displacement of the atoms from these equilibrium positions. A boundary to wave propagation occurs when there is a change in the mass of the atoms or in the strength of the spring force or at the termination of the chain. We introduce the following notation:

For the 1-D chain:

$U_{n}$ is the displacement (from equilibrium position) of particle $\mathrm{n}$.

$K$ is the spring constant (assuming a harmonic potential).

$m$ is the particle mass.

$a$ is the equilibrium spacing between particles.

For a wave propagating along the 1-D chain:

$A$ is the wave amplitude, $t$ is time, $k$ is angular wavenumber and $\omega$ is angular frequency.

\subsection{Equation of Motion and Dispersion Relation}

The force on particle $\mathrm{n}$ due to the 'spring' to its left is

$$
K U_{n-1}-K U_{n}
$$

Similarly the force on particle $\mathrm{n}$ due to the 'spring' to its right is

$$
K U_{n+1}-K U_{n}
$$

This gives the following equation of transverse or longitudinal motion for particle $\mathrm{n}$ (using $m a=\mathrm{F}$ ),

$$
m \ddot{U}_{n}=K\left(U_{n-1}-2 U_{n}+U_{n+1}\right) .
$$

Assume that a sinusoidal wave (travelling to the right) is a solution to the equation of motion, i.e. $U_{n}=A e^{i(\omega t-k a n)}$. The elements in Eq. (6.1) can be 
expressed in terms of $U_{n}$ as follows: $\ddot{U}_{n}=-\omega^{2} U_{n}, U_{n-1}=e^{i k a} U_{n}$ and $U_{n+1}=$ $e^{-i k a} U_{n}$. Substituting these into Eq. (6.1) and rearranging we obtain the following

$$
\omega=2 \sqrt{\frac{K}{m}}\left|\sin \left(\frac{k a}{2}\right)\right|
$$

We take the positive root as $\omega$ is a nonnegative quantity. The formula for $\omega^{2}$ is thus:

$$
\omega^{2}=2 \frac{K}{m}(1-\cos (k a)) .
$$

Equation (6.2) is the dispersion relation for the discrete 1-D chain, which expresses the frequency $\omega$ as a Function of the wave number $k$ and the material properties $K$ and $m$. Note that the dispersion relation can be rearranged to express $k$ as a Funtion of $\omega$.

For a fixed value of $a$, the dispersion relation only depends on the ratio $\sqrt{\frac{K}{m}}$. The graph of $\omega$ is shown in Figure 6.1. The graph for $\omega$ rises to a maximum of $2 \sqrt{\frac{K}{m}}$ at $k a=\pi$. This maximum frequency is a feature of waves on a discrete chain of this nature.

Note that the range of $k a$ is limited to $-\pi<k a<\pi$. A wave number of $\frac{\pi}{a}$ means the wave length equals $2 a$; twice the interatom spacing. Waves with wave length shorter than $2 a$ cannot be distinguished on the discrete chain. The motion of atoms on the chain for a wave length less than $2 a$ is indistinguishable from the motion due to some other wave length longer than $2 a$. This is incorporated in the dispersion relation whereby (due to the symmetry of $\sin \left(\frac{k a}{2}\right)$ about $\frac{\pi}{2}$ ) a wave number greater than $\frac{\pi}{a}$ gives the same frequency $\omega$ as some wave number smaller than $\frac{\pi}{a}$.

\subsection{Reflection of Waves}

If a propagating wave encounters a region with different material properties, i.e. a change in $K, m$ or $a$, then some portion of the wave will be reflected from the interface between the different regions. 


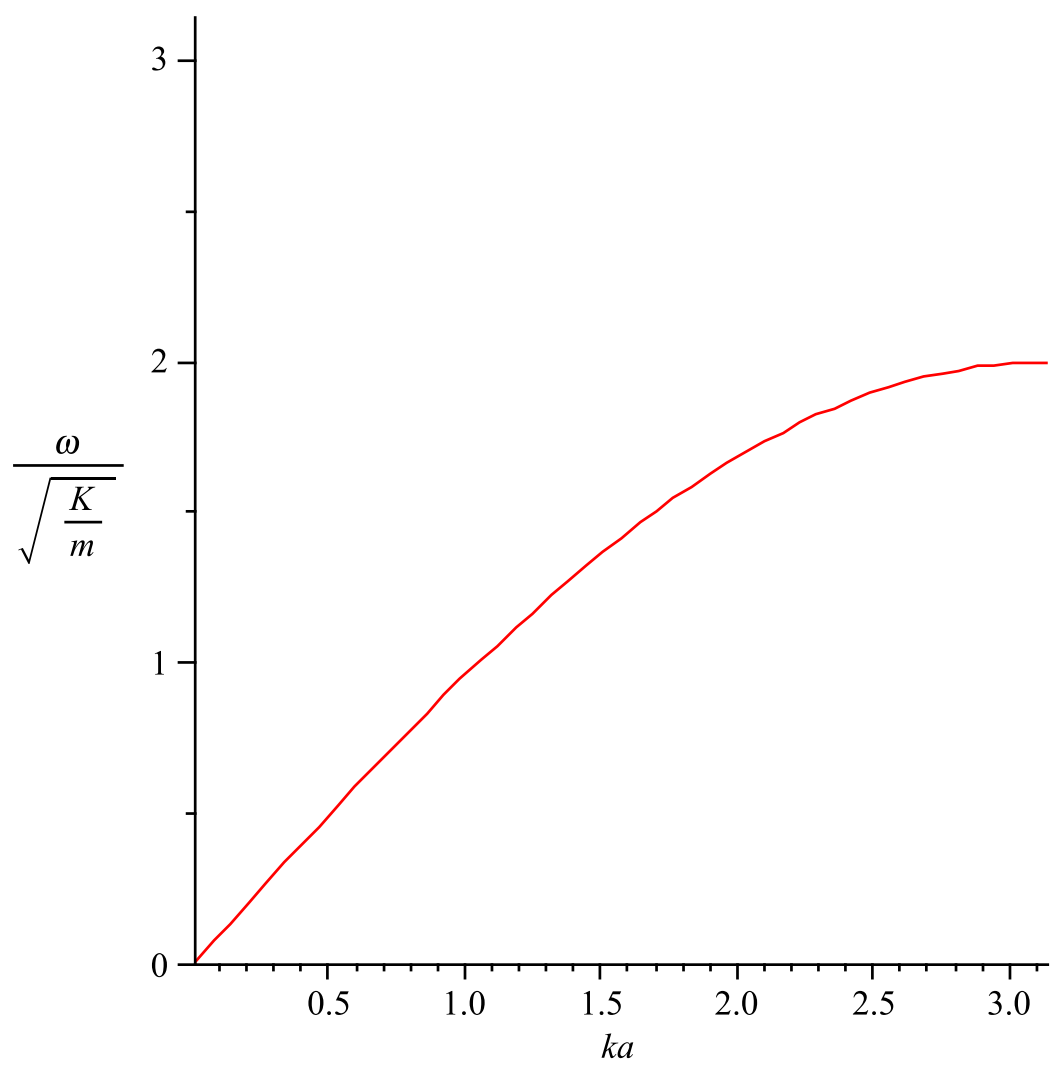

Figure 6.1: The dispersion relation for the discrete 1-D chain. In this figure $\sqrt{\frac{K}{m}}=$ $\sqrt{\frac{4}{4}}=1$.

\subsubsection{Reflection Coefficient $R_{c}$}

When a wave is reflected from an interface, the ratio of the amplitude of the reflected wave to that of the incident wave defines the reflection coefficient $R_{c}$.

Consider a 1-D discrete chain that exhibits a change in particle mass at $n=0$. Particles to the left of zero have mass $m$ and those at zero and rightwards have mass $M$. The equations of motion are then:

for $n \leq-1$

$$
m \ddot{U}_{n}=K\left(U_{n-1}-2 U_{n}+U_{n+1}\right)
$$


while for $n=0$

$$
M \ddot{U}_{0}=K\left(U_{-1}-2 U_{0}+U_{1}\right) .
$$

An incident wave with unit amplitude travels from the left to the right. At $n=0$ part of the incident wave is reflected back to the left and part continues to travel on to the right.

For $n \leq-1$ the displacement is a superposition of the incident and reflected waves, where $R_{c}$ is the amplitude of the reflected wave. We define two regions along the 1-D chain. Region $L$ is to the left of zero (i.e $n<0$ ) and region $R$ encompasses zero and rightwards (i.e. $n \geq 0$ ).

The displacement in region $L$ is given by the following equation

$$
U_{n}=e^{i\left(\omega t-k_{L} a n\right)}+R_{c} e^{i\left(\omega t+k_{L} a n\right)} .
$$

Note that the equation for the reflected (leftward travelling) wave differs from the equation of the incident (rightward travelling) wave by the change of the sign of the wave number $k_{L}$ in region $L$, and by multiplication by the reflection coefficient $R_{c}$.

At $n=0$ the displacement due to the superposition of the incident and reflected waves must equal the displacement due to the transmitted wave:

$$
U_{n}=e^{i\left(\omega t-k_{L} a n\right)}+R_{c} e^{i\left(\omega t+k_{L} a n\right)}=T_{c} e^{i\left(\omega t-k_{R} a n\right)}
$$

i.e.

$$
U_{0}=e^{i \omega t}+R_{c} e^{i \omega t}=T_{c} e^{i \omega t}
$$

hence $1+R_{c}=T_{c}$.

For $n>0$ the transmitted wave moves to the right:

$$
U_{n}=T_{c} e^{i\left(\omega t-k_{R} a n\right)}
$$

A smooth transmission of waves across the interface requires that the slope of the displacement of the superposition of waves for $n \leq 0$ is equal to the slope of the displacement for the transmitted wave for $n \geq 0$ at $n=0$. The respective slopes (derivatives with respect to distance $n$ ) are:

$$
\left.\frac{d U_{n}}{d n}\right|_{L}=-k_{L} a e^{i\left(\omega t-k_{L} a n\right)}+k_{L} a R_{c} e^{i\left(\omega t+k_{L} a n\right)}
$$


and

$$
\left.\frac{d U_{n}}{d n}\right|_{R}=-k_{R} a T_{c} e^{i\left(\omega t-k_{R} a n\right)} .
$$

Equating these at $n=0$ gives:

$$
-k_{L} a e^{i \omega t}+k_{L} a R_{c} e^{i \omega t}=-k_{R} a T_{c} e^{i \omega t}
$$

Canceling the $a$ and $e^{i \omega t}$ terms and using the fact that $1+R_{c}=T_{c}$, this equation then becomes

$$
-k_{L}+k_{L} R_{c}=-k_{R}\left(1+R_{c}\right) .
$$

Note that we are equating a wave with frequency $\omega$ for $n \leq 0$ with a wave transmitted past $n=0$ that also has frequency $\omega$. We assume that the interatomic spacing $a$ is the same for all values of $n$.

Solving for $R_{c}$ now gives the following equation for the reflection coefficient in terms of the wave numbers in the two regions $\left(k_{L}\right.$ in region $L$ and $k_{R}$ in region $R)$ :

$$
R_{c}=\frac{k_{L}-k_{R}}{k_{L}+k_{R}}
$$

From this equation it follows that when there is no difference between region $L$ and region $R$, i.e. $K$ and $m$ are the same in both regions, their respective wave numbers $k_{L}$ and $k_{R}$ will be the same. The reflection coefficient $R_{c}$ will then be zero, and the transmission coefficient $T_{c}$ will be equal to 1 in this instance. As the difference between the regions gets larger, so does the difference between their respective wave numbers and consequently $\left|R_{c}\right|$ (the magnitude of the reflection coefficient) increases, giving rise to larger amplitude reflected waves from the interface. When the magnitude of $k_{R}$ exceeds that of $k_{L}, R_{c}$ becomes negative, indicating a $180^{\circ}$ phase shift (inversion) of the reflected wave relative to the incident wave.

\subsection{Dispersion Relation with Damping}

Adding a constant frictional damping coefficient $\gamma$ to each atom in the discrete 1-D chain gives the following equation of motion: 


$$
m \ddot{U}_{n}=K\left(U_{n-1}-2 U_{n}+U_{n+1}\right)-\gamma \dot{U}_{n} .
$$

Where the effect on atom $n$, from the damping applied to atoms $n-1$ and $n+1$, feeds through in their altered displacements (compared to the undamped case).

Again, for the same assumed sinusoidal wave solution, we have in addition that $\dot{U}_{n}=i \omega U_{n}$. Thus, following the derivation above gives the dispersion relation for this case of constant damping:

$$
\omega^{2}=4 \frac{K}{m} \sin ^{2}\left(\frac{k a}{2}\right)+i \omega \frac{\gamma}{m},
$$

i.e.

$$
\omega=\frac{i \omega \frac{\gamma}{m} \pm \sqrt{\left(-i \omega \frac{\gamma}{m}\right)^{2}+16 \frac{K}{m} \sin ^{2}\left(\frac{k a}{2}\right)}}{2} .
$$

We assume a complex wave number $k=k_{r e}-i k_{i m}$ because the real and imaginary parts of such a wave number play distinct roles. The real part $k_{r e}$ becomes the wave vector for the damped wave. The imaginary part $k_{i m}$ gives rise to exponential damping of wave amplitude with distance, with damping multiple $e^{-k_{i m} a n}$.

Using $k=k_{r e}-i k_{i m}$, we solve for $\omega$ as follows:

$$
\begin{aligned}
& \left.\omega^{2}=4 \frac{K}{m} \sin ^{2}\left(\frac{k_{r e} a-i k_{i m} a}{2}\right)\right)+i \omega \frac{\gamma}{m} \\
& =\frac{K}{m}\left(2-2 \cos \left(k_{r e} a-i k_{i m} a\right)\right)+i \omega \frac{\gamma}{m} .
\end{aligned}
$$

This can be split into its Real part

$$
\omega^{2}=2 \frac{K}{m}\left(1-\cosh \left(k_{i m} a\right) \cos \left(k_{r e} a\right)\right)
$$

and Imaginary part

$$
0=-2 \frac{K}{m}\left(\sinh \left(k_{i m} a\right) \sin \left(k_{r e} a\right)\right)+\omega \frac{\gamma}{m} .
$$

Solving for $\cosh \left(k_{i m} a\right)$ gives:

$$
\cosh \left(k_{i m} a\right)=\mathrm{C} \pm \mathrm{D}
$$


where

$$
\mathrm{C}=\frac{-\gamma^{2} \cos \left(k_{r e} a\right)}{4 K m\left(1-\cos ^{2}\left(k_{r e} a\right)\right)}
$$

and

$$
\mathrm{D}=\sqrt{\mathrm{C}^{2}+1+\frac{\gamma^{2}}{2 K m\left(1-\cos ^{2}\left(k_{r e} a\right)\right)}} .
$$

Note that as $K, m$ and $\left(1-\cos ^{2}\left(k_{r e} a\right)\right)$ are non-negative, all the terms inside the square root are also non-negative. Therefore $\mathrm{D}$ is real and positive.

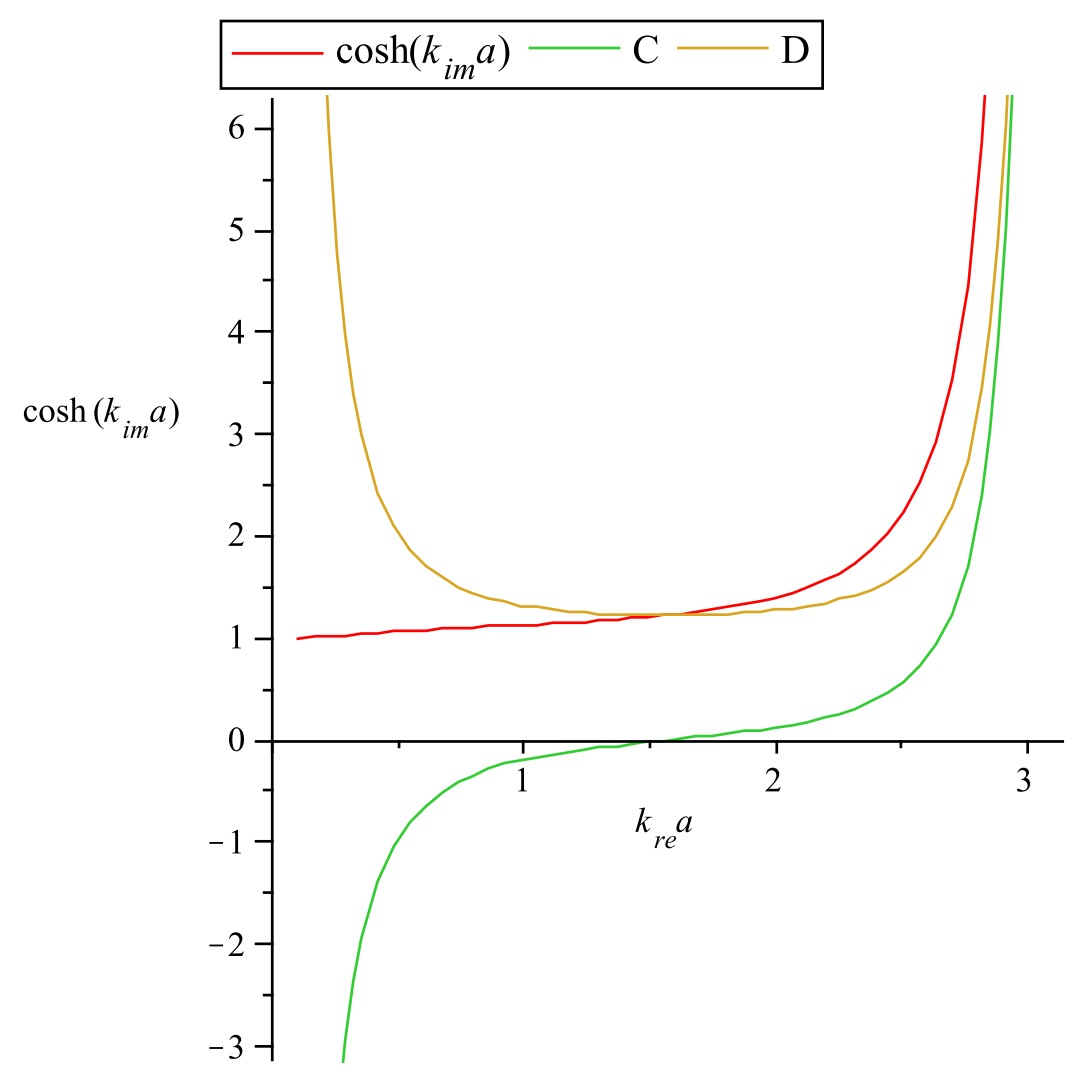

Figure 6.2: The red curve is $\cosh \left(k_{i m} a\right)$. It is formed by summation of the other two curves. 
$\mathrm{C}$ is negative overall for $k_{r e} a$ from 0 to $\frac{\pi}{2}$, because $\cos \left(k_{r e} a\right)$ is nonnegative over that range. So over this range $\cosh \left(k_{i m} a\right)=-|\mathrm{C}|+\mathrm{D}$ is less than $\mathrm{D}$. For $k_{r e} a>\frac{\pi}{2}, \mathrm{C}$ has positive sign and $\operatorname{so} \cosh \left(k_{i m} a\right)$ is the sum of both terms.

Figure 6.2 shows this construction of $\cosh \left(k_{i m} a\right)$ (red curve) from its two terms, when $K, m, a$, and $\gamma$ are all 1 . In this case $\cosh \left(k_{i m} a\right)$ is fairly flat for $k_{r e} a$ less than approximately 2 , after which $\cosh \left(k_{i m} a\right)$ rises steeply. When $\cosh \left(k_{i m} a\right) \approx$ 1 then $k_{i m} a \approx 0$ and there is very little damping (see section 6.5.2).

The rapid increase in $\cosh \left(k_{i m} a\right)$ as $k_{r e} a$ tends towards $\pi$ is due to the $\frac{1}{\left(1-\cos ^{2}\left(k_{r e} a\right)\right)}$ elements in both $\mathrm{C}$ and $\mathrm{D}$.

The imaginary part of the wave vector $k_{i m}$, has the same shape as $\cosh \left(k_{i m} a\right)$, but with a reduced magnitude and a minimum value of zero (when $k_{r e} a=0$ ).

In Figure 6.3 the effect of varying $\gamma$ on $\cosh \left(k_{i m} a\right)$ and hence $k_{i m}$ is shown. From the equation of $\cosh \left(k_{i m} a\right)$, it can be seen that this curve depends on the ratio $\frac{\gamma^{2}}{K m}$. This ratio is a dimensionless quantity, which is referred to as the damping number in this thesis. Two different sets of $\gamma, K$ and $m$, with the same damping number, have identical $\cosh \left(k_{i m} a\right)$ curves.

As $\gamma$ becomes very large (relative to $K$ and $m$ ), the graph of the First term in $\cosh \left(k_{i m} a\right)$ tends to a vertical line through $k_{r e} a=\frac{\pi}{2}$. This results in the graph of $\cosh \left(k_{i m} a\right)$ tending to infinity as $k_{r e} a$ approaches $\frac{\pi}{2}$, as displayed in Figure 6.3.

This equation for $\cosh \left(k_{i m} a\right)$ effectively expresses $k_{i m}$ in terms of $k_{r e}$. Therefore $\omega$ can now be evaluated in terms of $k_{r e}$ by substituting for $\cosh \left(k_{i m} a\right)$ in the Real part formula Eq. (6.6), repeated here:

$$
\omega^{2}=2 \frac{K}{m}\left(1-\cosh \left(k_{i m} a\right) \cos \left(k_{r e} a\right)\right)
$$

This is the same form as for $\omega^{2}$ in the undamped case Eq. (6.3), with the additional $\cosh \left(k_{i m} a\right)$ factor multiplying the $\cos \left(k_{r e} a\right)$ term. When $\gamma$ is zero, $\cosh \left(k_{i m} a\right)=1$ and $k_{i m}=0$, so that the usual dispersion relation for the 1-D discrete chain Eq. (6.2), is recovered.

By definition $\cosh \left(k_{i m} a\right)$ is greater than or equal to 1 , for all values of $k_{i m} a$. As $k_{r e} a$ increases from 0 to $\frac{\pi}{2}$, the $\cos \left(k_{r e} a\right)$ term declines from 1 to 0 . Therefore for $0<k_{r e} a<\frac{\pi}{2}$, the damped $\omega^{2}$ is less than or equal to the undamped case, with 


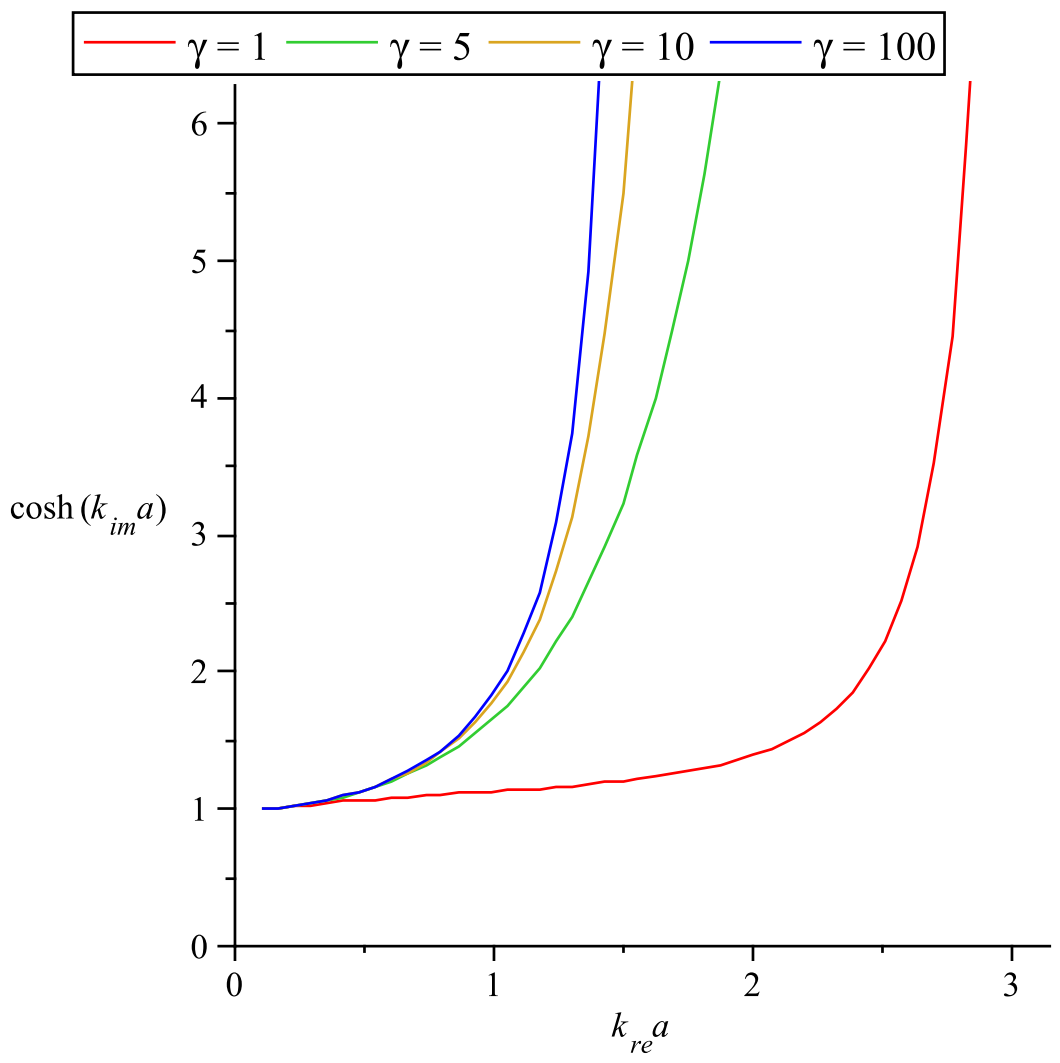

Figure 6.3: $\cosh \left(k_{i m} a\right)$ for a range of $\gamma$ values.

equality at $k_{r e} a=\frac{\pi}{2}$. Over the range $\frac{\pi}{2}<k_{r e} a \leq \pi$ the $\cos \left(k_{r e} a\right)$ term is negative, finally reaching -1 . Hence over this range $-\cosh \left(k_{i m} a\right) \cos \left(k_{r e} a\right)>0$, and so the damped $\omega^{2}$ exceeds the undamped case.

When $k_{r e} a \approx \pi$, then $\cos \left(k_{r e} a\right) \approx-1$, so that the damped $\omega^{2}$ is:

$$
\omega^{2} \approx 2 \frac{K}{m}\left(1+\cosh \left(k_{i m} a\right)\right) .
$$

Figure 6.4 shows the damped 1-D dispersion relation i.e. $\omega$ versus $k_{r e} a$ curves, for a range of $\gamma$ values. These curves follow from the corresponding $\cosh \left(k_{i m} a\right)$ curves discussed previously. For a fixed $a$, the damped dispersion relation only depends on $\sqrt{\frac{K}{m}}$ and the damping number. The undamped case (red curve), where $\gamma=0$, rises to reach its maximum value $2 \sqrt{\frac{K}{m}}$ when $k_{r e} a=\pi$, as seen previously 
in Figure 6.1. Note that in contrast to the undamped case discussed previously, there is no maximum value of $\omega$ in the damped case. As $\gamma$ increases (relative to $K$ and $m$ ), damped $\omega$ falls further below the undamped case when $k_{r e} a<\frac{\pi}{2}$. All the graphs intersect when $k_{r e} a=\frac{\pi}{2}$. Conversely for $k_{r e} a>\frac{\pi}{2}$ the damped curve progressively exceeds the undamped case with increasing $\gamma$.

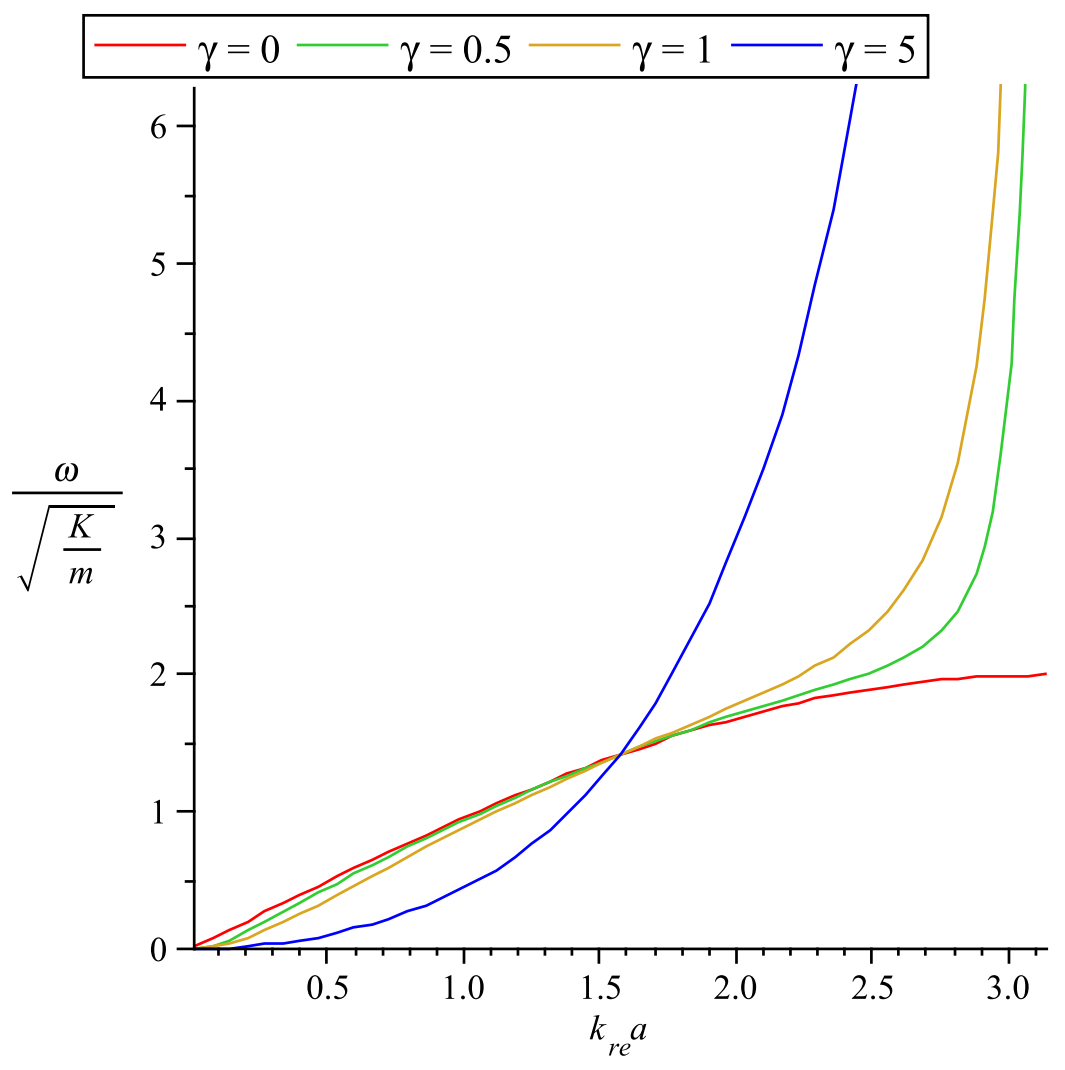

Figure 6.4: The damped 1-D dispersion relation. This figure shows $\omega$ as a function of $k_{r e} a$, for a range of $\gamma$ values. Here $\sqrt{\frac{K}{m}}=\sqrt{\frac{4}{4}}=1$. 


\subsection{1 $k$ as a Function of $\omega$}

The dispersion relation derived previously enables determination of $\omega$ for given $k$. The reflection coefficient $R_{c}$ depends on the values of $k$ in the respective regions. Therefore we rearrange the dispersion relation to express $k$ as a function of $\omega$.

First we effectively solve for $k_{r e}$ by developing a formula for $\cos \left(k_{r e} a\right)$. A simple rearrangement of the Real part formula Eq. (6.6) gives:

$$
\cosh \left(k_{i m} a\right) \cos \left(k_{r e} a\right)=1-\frac{1}{2} \omega^{2} \frac{m}{K} .
$$

Substitute for $\cosh \left(k_{i m} a\right)$ to obtain an equation in $\cos \left(k_{r e} a\right)$

$$
\begin{gathered}
\frac{-\gamma^{2} \cos \left(k_{r e} a\right)^{2}}{4 K m\left(1-\cos \left(k_{r e} a\right)^{2}\right)}+ \\
\sqrt{\frac{\gamma^{4} \cos \left(k_{r e} a\right)^{4}}{4 K^{2} m^{2}\left(1-\cos \left(k_{r e} a\right)^{2}\right)^{2}}+\cos \left(k_{r e} a\right)^{2}+\frac{\gamma^{2} \cos \left(k_{r e} a\right)^{2}}{2 K m\left(1-\cos \left(k_{r e} a\right)^{2}\right)}} \\
=1-\frac{1}{2} \omega^{2} \frac{m}{K} .
\end{gathered}
$$

Solving for $\cos \left(k_{r e} a\right)$ gives the following four solutions

$$
\cos \left(k_{r e} a\right)= \pm \frac{\sqrt{2}}{4 K} \sqrt{\mathrm{P} \pm \sqrt{\mathrm{Q}}}
$$

Where

$$
\mathrm{P}=\omega^{4} m^{2}+\left(\gamma^{2}-4 K m\right) \omega^{2}+8 K^{2}
$$

and

$$
\mathrm{Q}=\omega^{8} m^{4}+\left(2 m^{2} \gamma^{2}-8 m^{3} K\right) \omega^{6}+\left(4 K m-\gamma^{2}\right)^{2} \omega^{4}+16 K^{2} \omega^{2} \gamma^{2}
$$

The first two solutions,

$$
\pm \frac{\sqrt{2}}{4 K} \sqrt{\mathrm{P}+\sqrt{\mathrm{Q}}}
$$

are rejected as they give $\left|\cos \left(k_{r e} a\right)\right|>1$. 
We now show that both of the remaining solutions

$$
\mathrm{S}_{+}=\frac{\sqrt{2}}{4 K} \sqrt{\mathrm{P}-\sqrt{\mathrm{Q}}}
$$

and

$$
\mathrm{S}_{-}=-\frac{\sqrt{2}}{4 K} \sqrt{\mathrm{P}-\sqrt{\mathrm{Q}}}
$$

need to be used, to obtain the correct formula for $\cos \left(k_{r e} a\right)$ (and hence $k_{r e}$ ) in terms of $\omega$ and the other parameters.

Figure 6.5 shows the values of $k_{r e} a$ as a function of $\omega$, given by each of $\mathrm{S}_{+}$ (red curve) and $\mathrm{S}_{-}$(green curve) in the simple case of no damping.

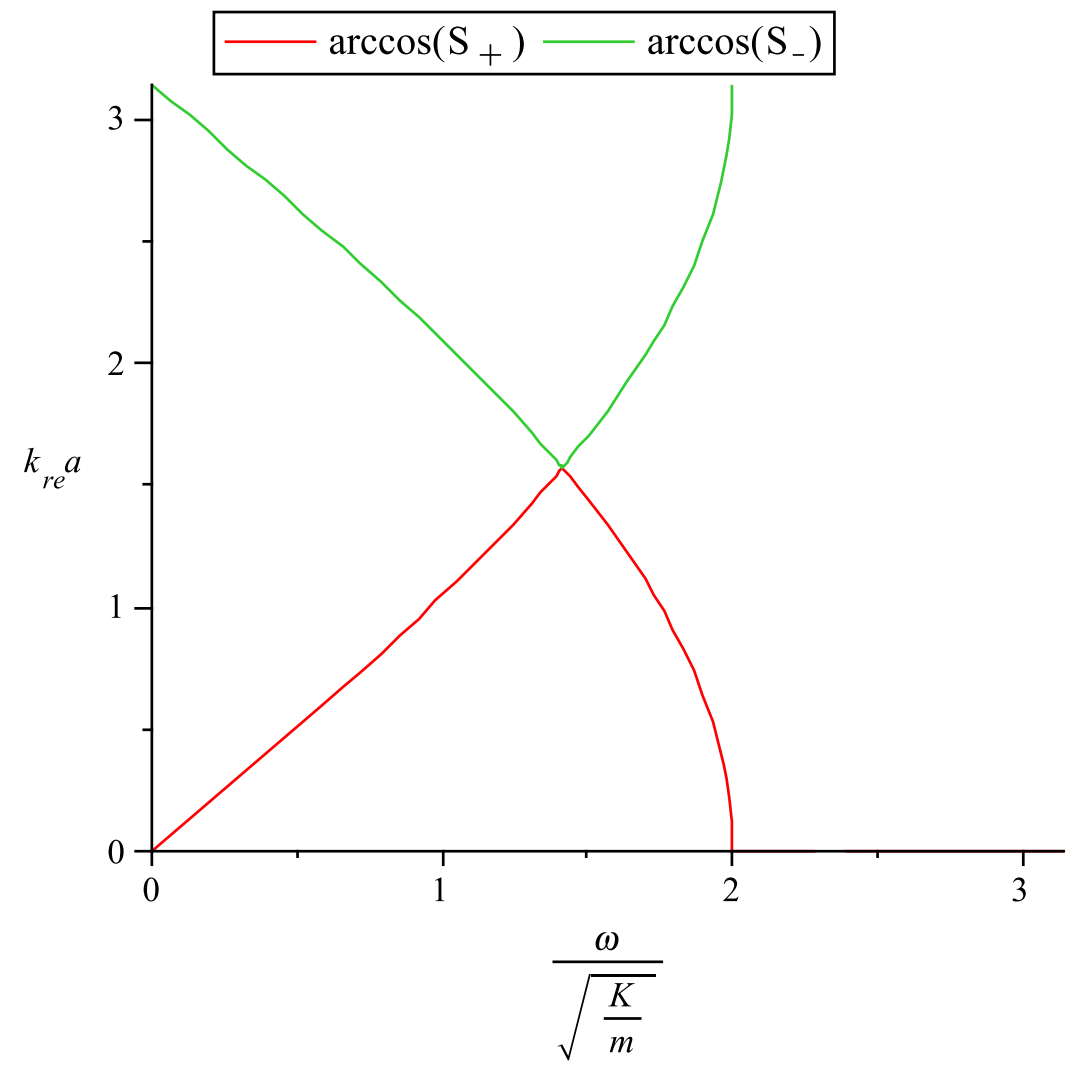

Figure 6.5: This figure shows $k_{r e} a$ as a function of $\omega$ from $\mathrm{S}_{+}$and from $\mathrm{S}_{-}$. In this figure $\sqrt{\frac{K}{m}}=1$. 
The correct relationship between $k_{r e} a$ and $\omega$, under the conditions in Figure 6.5, is the undamped dispersion relation plotted in Figure 6.1. Comparison of these two figures shows that in order to obtain the correct relationship, both solutions are required. $S_{+}$must be used for small values of $\omega$ up until the point at which the two solutions meet. This point is found to be $\sqrt{\frac{2 K}{m}}$. For larger values of $\omega, \mathrm{S}_{-}$is to be used. This dependence on the two solutions can be expressed more concisely by the formula:

$$
\left|\cos \left(k_{r e} a\right)\right|=\frac{\sqrt{2}}{4 K} \sqrt{\mathrm{P}-\sqrt{\mathrm{Q}}} .
$$

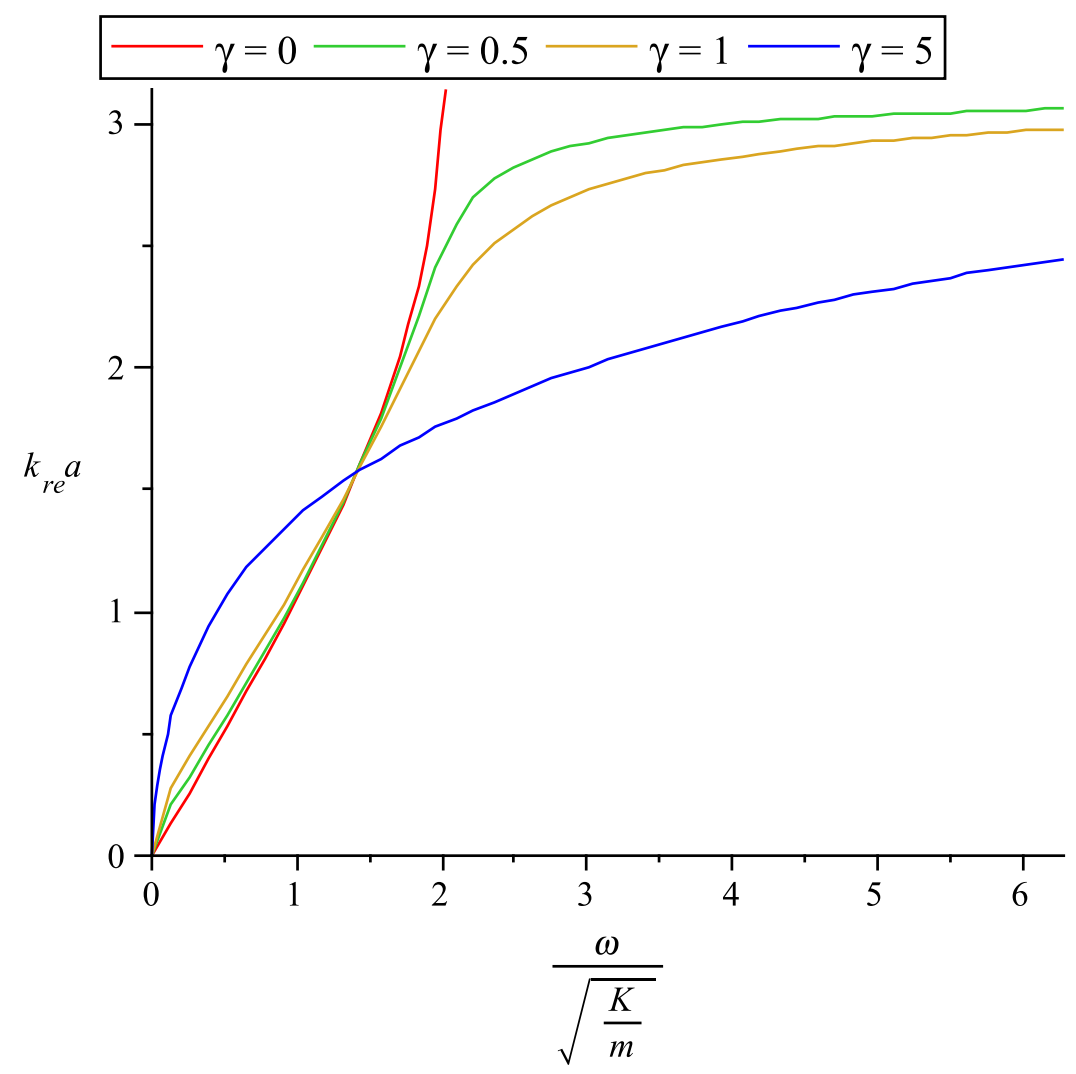

Figure 6.6: This figure shows $k_{r e} a$ as a function of $\omega$ for a range of values of $\gamma$, where $\sqrt{\frac{K}{m}}=1$.

The functional relationship of $k_{r e} a$ upon $\omega$ using both $\mathrm{S}_{+}$and $\mathrm{S}_{-}$, i.e. Eq. 
(6.8), is shown in Figure 6.6. This Figure displays the same relationship as in Figure 6.1, but now with $\omega$ as the independent variable.

The second step is to obtain the functional dependence of $k_{i m} a$ on $\omega$. This is easily achieved using $\cosh \left(k_{i m} a\right)$, which is a function of $\cos \left(k_{r e} a\right)$, and therefore a function of $\omega$.

In Figure 6.7 we show the dependence of $k_{r e} a$ on $\omega$ for a larger range of $\gamma$ values. Figure 6.8 shows the comparable graphs for $k_{i m} a$.

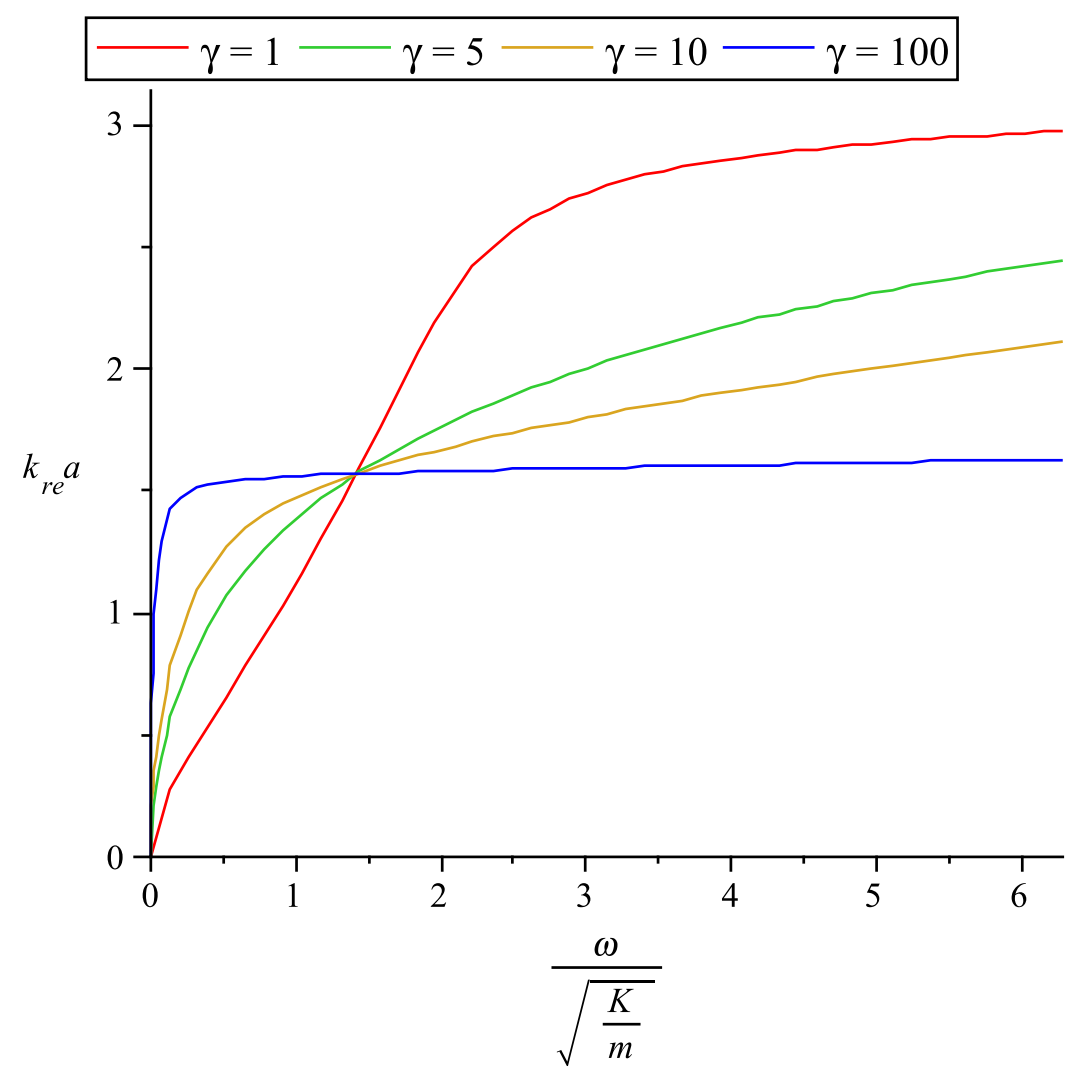

Figure 6.7: This figure shows $k_{r e} a$ as a function of $\omega$ for a broad range of values of $\gamma$. In this figure $\sqrt{\frac{K}{m}}=1$. 


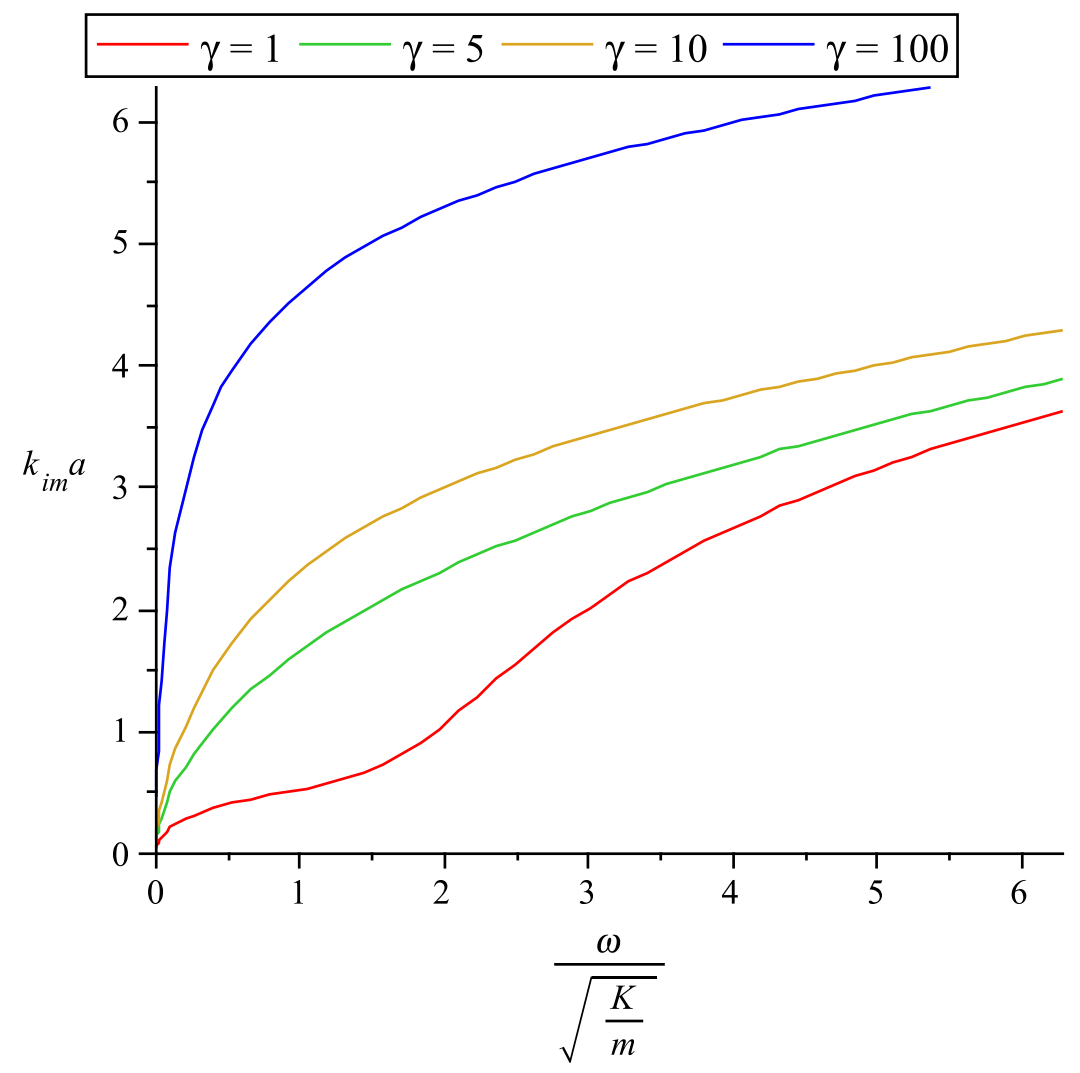

Figure 6.8: This figure plots $k_{i m} a$ as a function of $\omega$ for a broad range of values of $\gamma \cdot \sqrt{\frac{K}{m}}=1$.

\subsubsection{Damping Effect}

With complex wave number $k=k_{r e}-i k_{i m}$, the displacement for a particle on the 1-D chain becomes:

$$
U_{n}=A e^{i\left(\omega t-\left(k_{r e}-i k_{i m}\right) a n\right)}=e^{-k_{i m} a n} A e^{i\left(\omega t-k_{r e} a n\right)} .
$$

As noted earlier, $k_{r e}$ is the wave vector for the damped wave and $k_{i m}$ gives the damping multiple $e^{-k_{i m} a n}$. The size of $k_{i m} a$ determines the magnitude of the damping in wave amplitude over the distance $a$ (one interparticle spacing). As $k_{i m} a$ increases, the damping multiple decreases exponentially, and a given wave 
amplitude is extinguished over a shorter distance.

The dependence of $k_{i m} a$ on $\omega$, for a broad range of damping parameters $\gamma$, is shown in Figure 6.8.

The following features are of note. When $\omega$ is very close to zero, $k_{i m} a$ is effectively zero and thus there is no damping of very low frequency waves. For such small values of $\omega, k_{r e}$ is also very small; see Figure 6.7. Therefore these low frequencies correspond to wave lengths that are extremely long with respect to the interatom spacing $a$. As $\omega$ increases for a given value of $\gamma$, the value of $k_{i m} a$ initially rises rapidly, before reducing to a slower growth rate. For an increased $\gamma$, this rapid rise occurs at a smaller value of $\omega$. Not shown in Figure 6.8 is the case of $\gamma=0$, where $k_{i m} a$ is zero and there is no damping. For a given value of $\gamma$, $k_{i m} a$ increases as $\omega$ increases. Thus, high frequency waves have their amplitudes heavily damped and only propagate short distances before being extinguished. For example, when $\gamma=1$ and $\omega \approx 6$, then $k_{i m} a \approx 3.5$. The corresponding damping multiple over the distance of a single interatomic space (when $a=1$ ) is $e^{-3.5} \approx 0.03$. Therefore in the case of $\gamma=1$, a wave with $\omega \approx 6$ has its amplitude damped down to $\approx 3 \%$ of its previous value, over a distance of one interatomic space.

\subsubsection{Damped Reflection Coefficient}

Here we consider reflections from the interface between two regions, both of which (in the most general case) have damping forces. As previously, the region to the left of the interface is labelled $L$, and the region to the right is labelled $R$. The previous derivation of the reflection coefficient still holds, but in this case the wave numbers in the damped regions are now complex numbers. In the case of the reflected wave, the sign of the real part of the complex wave number is inverted to give a wave travelling to the left. If both regions are damped, the formula for the reflection coefficient $R_{c}$ becomes

$$
R_{c}=\frac{\left(k_{\text {Lre }}-i k_{\text {Lim }}\right)-\left(k_{R r e}-i k_{\text {Rim }}\right)}{\left(k_{\text {Lre }}+i k_{\text {Lim }}\right)+\left(k_{\text {Rre }}-i k_{\text {Rim }}\right)} .
$$


Thus, in the case of damping in both regions (or only one region), the reflection coefficent $R_{c}$ becomes a complex number.

Clearly when there is no damping in either region (zero imaginary components of the wave numbers) this formula reduces to the previous (undamped) formula and $R_{c}$ is purely real.

The complex reflection coefficient $R_{c}$ can be expressed in polar form:

$$
R_{c}=\left|R_{c}\right| e^{i \operatorname{Arg}\left(R_{c}\right)}
$$

When a wave is reflected its wave form is multiplied by $R_{c}$. Therefore the amplitude of the wave is multiplied by $\left|R_{c}\right|$ and the phase of the wave is changed by $\operatorname{Arg}\left(R_{c}\right)$. In the previous undamped case, only amplitude variations (and complete inversion) could occur.

This reflection coefficient and the corresponding transmission coefficient provide the amplitude (and phase change) of the reflected and transmitted waves, respectively, at the interface. As these waves travel, their amplitudes are reduced with distance travelled, due to the corresponding damping multiple $e^{-k_{i m} a n}$.

Consider a wave of a given frequency $\omega$ travelling in region $L$ towards region $R$. In the general case at the interface between region $L$ and region $R$, part of the wave is reflected back into region $L$ and part is transmitted into region $R$. In the particular case of the two regions having exactly the same material properties (i.e. same $K$ and $m$ ) and same damping forces (if any), the wave is fully transmitted without any reflection, giving $\left|R_{c}\right|=0$. A more general statement can also be made. The dispersion relation in a region, and hence the values of $k_{r e}$ and $k_{i m}$, only depends on the ratio $\frac{K}{m}$ and the damping number $\frac{\gamma^{2}}{K m}$ in the region. Consequently, when each of the pair of regions has the same ratio $\frac{K}{m}$ and the same damping number, there will be no reflections from the interface between them.

In order to determine the reflection coefficient $R_{c}$ for a wave of a given frequency $\omega$ using the formula presented above, the complex wave numbers $k_{L}$ and $k_{R}$ in the two regions are required. These are determined using the formulas previously derived, and the relevant material values and damping parameter values in the respective regions. 


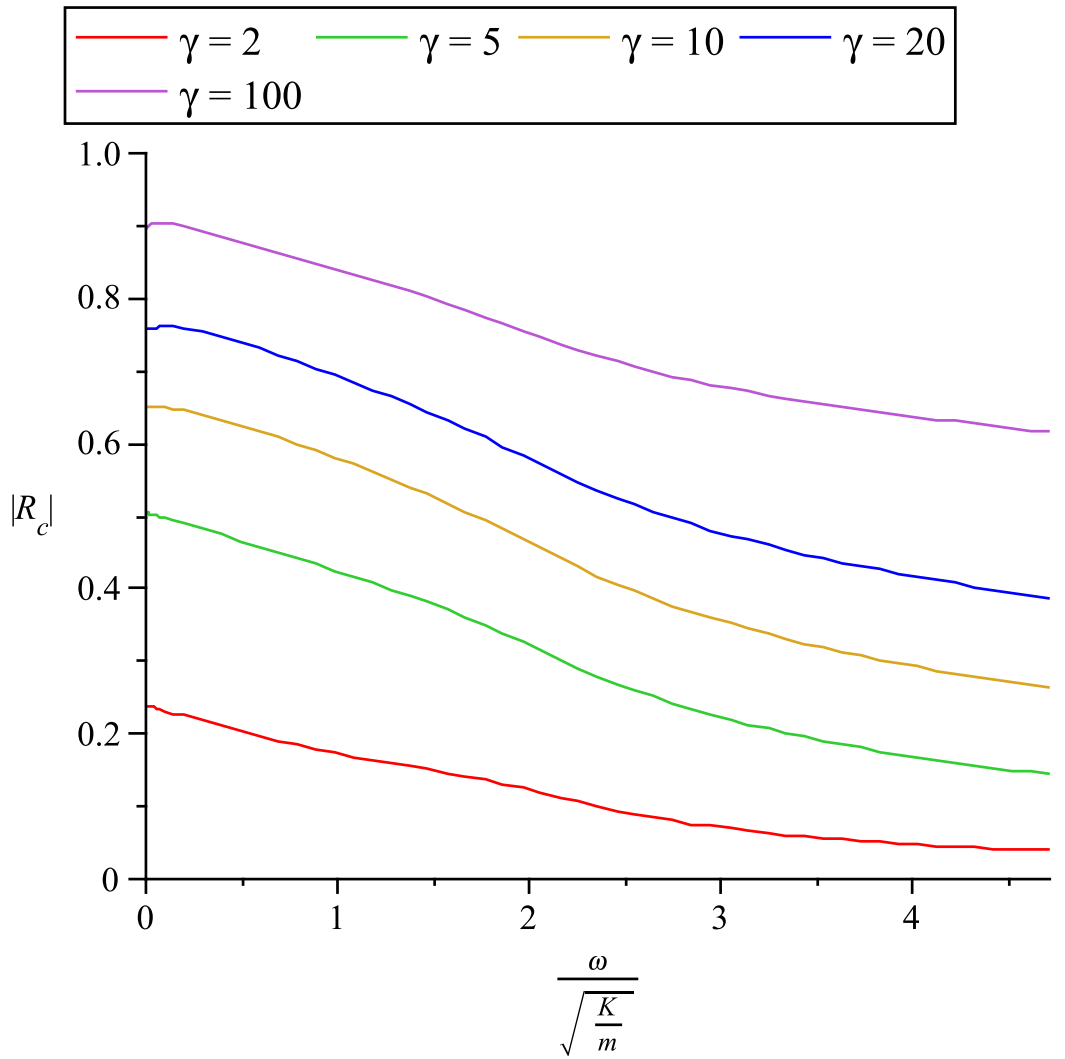

Figure 6.9: This figure shows $\left|R_{c}\right|$ as a function of $\omega$ for $\gamma=1$ in region $L$, and a range of $\gamma$ values in region $R \cdot \sqrt{\frac{K}{m}}=1$.

In Figure 6.9 there are two obvious trends. Firstly, for a given $\gamma$ the reflectivity $\left|R_{c}\right|$ decreases as $\omega$ increases. The decrease in $\left|R_{c}\right|$ is steeper for low values of $\omega$ and flattens out as $\omega$ gets larger. Secondly, for a given frequency $\omega$ the reflectivity $\left|R_{c}\right|$ increases as $\gamma$ increases. Increasing $\gamma$ in region $R$ makes a greater difference between the regions, when $\gamma=1$ is fixed in region $L$. 


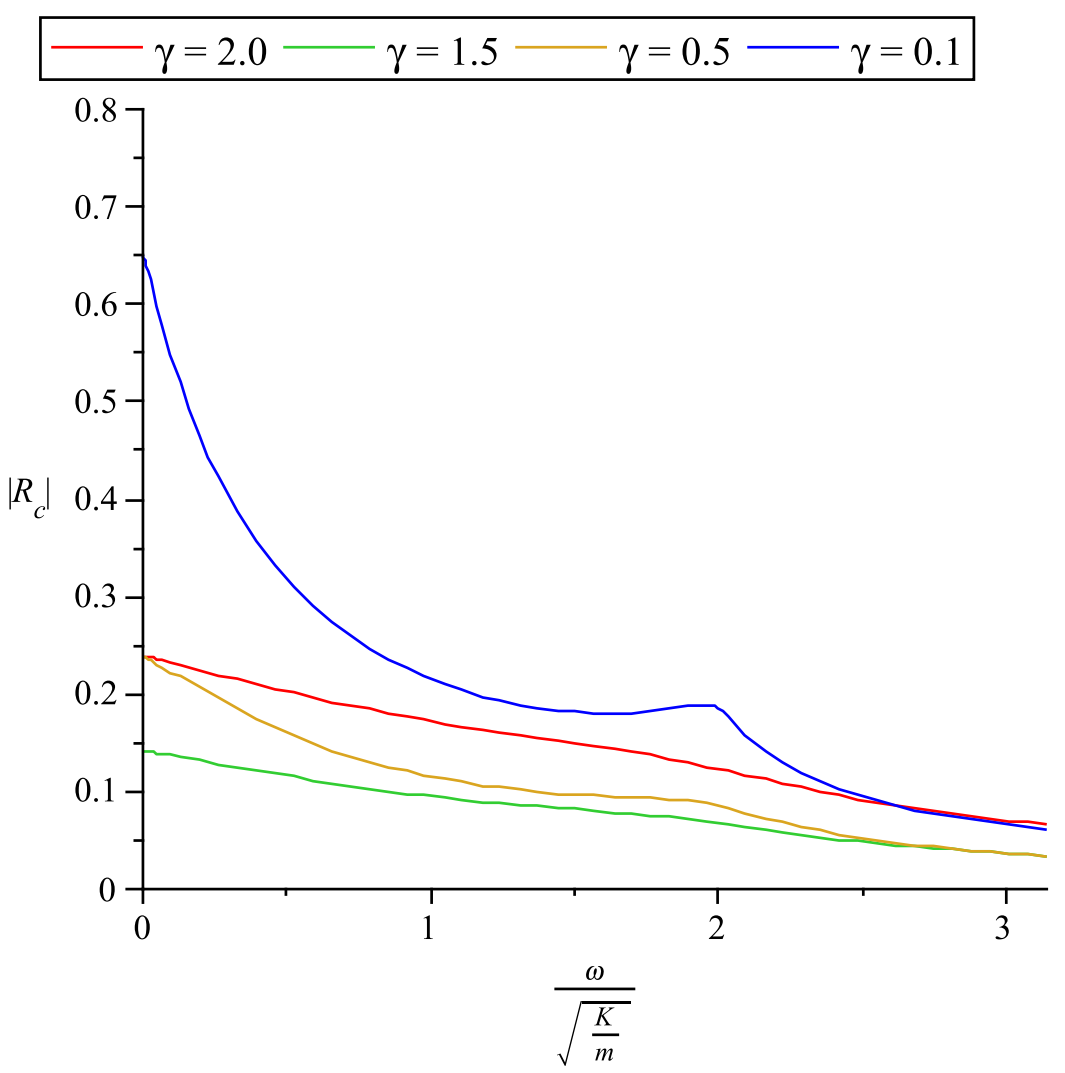

Figure 6.10: This figure shows $\left|R_{c}\right|$ as a function of $\omega$ for $\gamma=1$ in region $L$ and a range of small values of $\gamma$ in region $R \cdot \sqrt{\frac{K}{m}}=1$.

The case of reflections when $\gamma$ in region $R$ becomes less than $\gamma$ in region $L$, is shown in Figure 6.10. As the value of $\gamma$ in region $R$ reduces toward the value of $\gamma$ in region $L$ i.e. 1 , the value of $\left|R_{c}\right|$ decreases throughout the range of $\omega$. However once the value of $\gamma$ in region $R$ goes below 1 , the value of $\left|R_{c}\right|$ increases, particularly at smaller values of $\omega$. This again exhibits the fact that the greater the difference in $\gamma$ between the regions, the greater $\left|R_{c}\right|$, assuming all other parameters remain unchanged.

When $\gamma$ in region $R$ becomes very small, e.g. $\gamma=0.1$ (blue curve), the graph of $\left|R_{c}\right|$ begins to exhibit a hollow followed by a hump. This arises because for very small values of $\gamma$ the dispersion relation is very close to the undamped dispersion 
relation. In such cases $k_{R r e}$ follows the undamped $k_{R}$ curve until its value is close to $\pi$, whereupon $k_{R r e}$ very rapidly increases. This sudden increase in $k_{R r e}$ gives rise to the hump. As $\gamma$ in region $R$ declines further, the hump in $\left|R_{c}\right|$ concentrates into a cusp and its location moves towards $\omega=2 \sqrt{\frac{K}{m}}$, the maximum value of $\omega$ in the undamped case.

\subsection{Reflection with a Damping Region}

Here we investigate the use of a damping region, in which wave amplitude is reduced, resulting in dimished reflections back into the remainder of the MD region.

A damping region is formed in an MD region adjacent to the MD KMC interface. The damping region crosses the full width of the slab, and extends for a few rows of atoms along the axis of the slab away from the KMC region. The MD atoms in the damping region are subject to a damping force of the same form as, but different magnitude to, the Langevin damping parameter $\gamma$ used for temperature control.

Because of the rigidity of the KMC atoms, any waves reaching the MD KMC interface cannot be transmitted and must be fully reflected. Also as the transmitted wave amplitude is zero, the net amplitude of the incident and reflected wave at the interface must also be zero. Therefore the reflected wave must be out of phase with the incident wave, and hence $R_{c}=-1$ at the MD KMC interface.

To model a damping region between an MD region and a KMC region, the case discussed in the previous section of damping in both regions, with $\gamma=1$ in region $L$, is taken a step further. The additonal step is the introduction of a totally reflecting barrier located inside region $R$. This barrier is sited a distance $\mathrm{D}$ to the right of the interface, between region $L$ and region $R$. Region $L$ models the normal $\mathrm{MD}$ region, while the part of region $\mathrm{R}$ to the left of the barrier corresponds to the damping region. The barrier itself represents the MD KMC interface. When there is no difference in material properties and damping parameters between region $L$ and region $R$, there is no reflection from the interface between them. Also, there is no additional damping in region $R$. Increasing the damping in region $R$ will 
create reflections from the interface between region $L$ and region $R$, so waves will only be partly transmitted into region $R$ and then exposed to damping.

Minimising reflections of waves back into the MD region requires a large value of the damping parameter. However a large damping parameter will result in large reflections from the interface between region $L$ and region $R$, reducing the portion of the wave amplitude subject to damping. A balance needs to be struck between these two effects.

A further feature of region $R$ (the damping region), when damping is in effect, is partial internal reflection of waves inside region $R$. The portion of a wave that enters region $R$ and is then reflected (and fully inverted) by the barrier then undergoes a partial reflection at the interface between region $R$ and region $L$. The wave may traverse region $R$ several times (being damped on each traverse), transmitting a portion back into region $L$ each time it reaches the interface between region $R$ and region $L$, until the remnant of the wave inside region $R$ has been extinguished.

The resulting Net Reflectivity $(N R)$ back into the MD region of a wave that travels into the damping region and then undergoes a sequence of partial internal reflections (within the damping region) is now derived.

We introduce the following notation:

$R_{c L R}$ is the reflection coefficient for reflection from the interface between the MD region and the damping region, of a wave travelling (from left to right) into the damping region. The formula for $R_{c L R}$ is the same as the formula for $R_{c}$ derived previously.

$T_{c L R}=1+R_{c L R}$ is the transmission coefficient for transmission through the interface between the MD region and the damping region, of a wave travelling (from left to right) into the damping region.

$R_{c B}=-1$ is the reflection coefficient for reflection from the interface between the damping region and the $\mathrm{KMC}$ region i.e. reflection from the totally reflecting barrier.

$R_{c R L}$ is the reflection coefficient for reflection from the interface between the damping region and the MD region of a wave travelling (from right to left) out 
of the damping region. When $R_{c R L} \neq 0$, partial internal reflection (within the damping region) occurs.

$T_{c R L}=1+R_{c R L}$ is the transmission coefficient for transmission through the interface between the damping region and the MD region of a wave travelling (from right to left) out of the damping region.

$d_{k D}$ is the damping multiple of wave amplitude for a traverse of the damping region. The damping multiple depends on the value of the imaginary part of the wave number in the damping region, and on the number of rows of atoms in the damping region. 


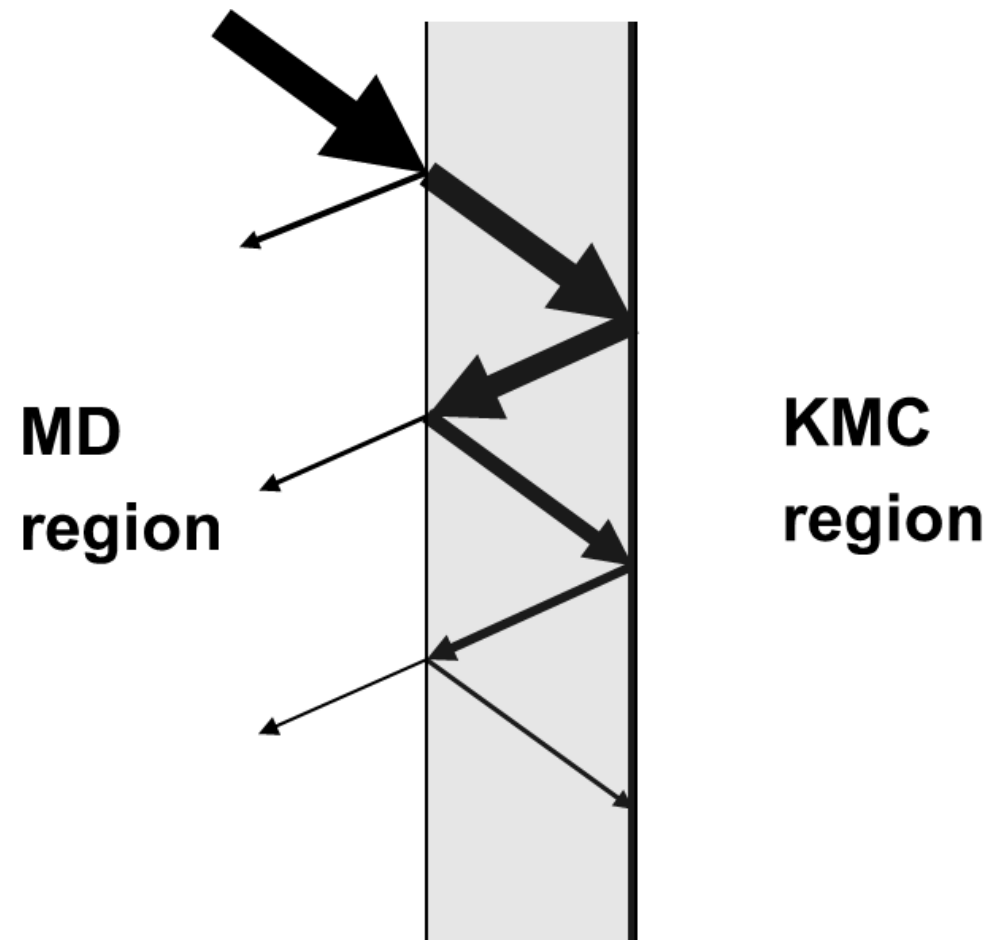

Figure 6.11: This diagram shows a wave (large arrow at top of diagram) travelling from the left out of the MD region into and through the MD damping region (shaded grey) and then reflecting off the interface (thick vertical line) between the MD damping region and $\mathrm{KMC}$ region. The wave is damped (indicated by the reduction in the size of the arrow) on each passage through the MD damping region. At the inteface (thin vertical line) between the MD damping region and the MD region there is both transmission (leftward out into the MD region) and reflection (rightward back into the MD damping region) of the wave. The part of the wave that is internally reflected within the MD damping region is eventually totally damped out.

For a wave of unit amplitude entering the damping region, the net reflectivity back into the MD region is composed of a number of components:

$R_{c L R}$ Reflection from the MD damping region interface.

$T_{c L R}\left(d_{k D} R_{c B} d_{k D}\right) T_{c R L}$ Transmission back into the MD region after a single damping cyle. 
$T_{c L R}\left(d_{k D} R_{c B} d_{k D}\right) R_{c R L}\left(d_{k D} R_{c B} d_{k D}\right) T_{c R L}$ Transmission after two damping cyles.

$$
T_{c L R}\left(d_{k D} R_{c B} d_{k D}\right) R_{c R L}\left(d_{k D} R_{c B} d_{k D}\right) R_{c R L}\left(d_{k D} R_{c B} d_{k D}\right) T_{c R L}
$$

Transmission after three damping cyles.

$\cdots$

$$
T_{c L R}\left(d_{k D} R_{c B} d_{k D}\right)^{m} R_{c R L}^{m-1} T_{c R L} \text { Transmission after m damping cyles. }
$$

Thus the net reflectivity back into the MD region is given by the following summation

$$
R_{c L R}+T_{c L R}\left(d_{k D} R_{c B} d_{k D}\right) T_{c R L} \sum_{m=0}^{\infty}\left(d_{k D} R_{c B} d_{k D} R_{c R L}\right)^{m}
$$

With damping in effect in the damping region, $\left|R_{c R L}\right|<1$ and $\left|d_{k D}\right|<1$, so the summation is a convergent geometric series. The net reflectivity is therefore

$$
N R=R_{c L R}+\frac{T_{c L R} d_{k D}^{2} R_{c B} T_{c R L}}{1-d_{k D}^{2} R_{c B} R_{c R L}} .
$$

From the definitions of $T_{c L R}, R_{c B}$ and $T_{c R L}$ this simply rearranges to

$$
N R=R_{c L R}-\left(1+R_{c L R}\right) d_{k D}^{2} \frac{1+R_{c R L}}{1+d_{k D}^{2} R_{c R L}} .
$$

Note that if the damping effect is set to zero then $d_{k D}=1$ and this formula reduces to

$$
R_{c L R}-\left(1+R_{c L R}\right)=-1
$$

Clearly when there is no damping effect in the damping region, all waves enter the region, are reflected and inverted by reflection from the MD KMC interface. They are then fully transmitted back into the MD region, undamped but inverted. 
The formula for $R_{c R L}$, the reflection coefficient for a wave travelling (right to left) from the damping region back into the MD region, is derived in the same way as the formula for the reflection coefficient $R_{c}$ derived previously, where $R_{c}$ is for waves travelling (left to right) from region $L$ into region $R$.

In the case of $R_{c R L}$, the incident wave and the portion of it that is transmitted travel from right to left, while the portion of the wave that is reflected travels to the right. To obtain leftward travelling incident and transmitted waves, the sign of the real part of the complex wave number for these two waves is inverted. The resulting formula is

$$
R_{c R L}=\frac{\left(k_{\text {Rre }}+i k_{\text {Rim }}\right)-\left(k_{\text {Lre }}+i k_{\text {Lim }}\right)}{\left(k_{\text {Rre }}-i k_{\text {Rim }}\right)+\left(k_{\text {Lre }}+i k_{\text {Lim }}\right)}
$$

Note that now the subscript $L$ (left region) refers to the MD region and the subscript $R$ (right region) refers to the damping region.

The formula for $d_{k D}$ is

$$
d_{k D}=e^{-k_{R i m} D / a} .
$$

The net reflectivity formula Eq. (6.9) results in a complex number. The modulus of this number gives the total amplitude of all the reflected components of the incident wave after damping. The argument of this complex number gives the aggregate phase change.

In Figure 6.12 we show the modulus of the net reflectivity $|N R|$, for a range of $\gamma$ values in the damping region. 


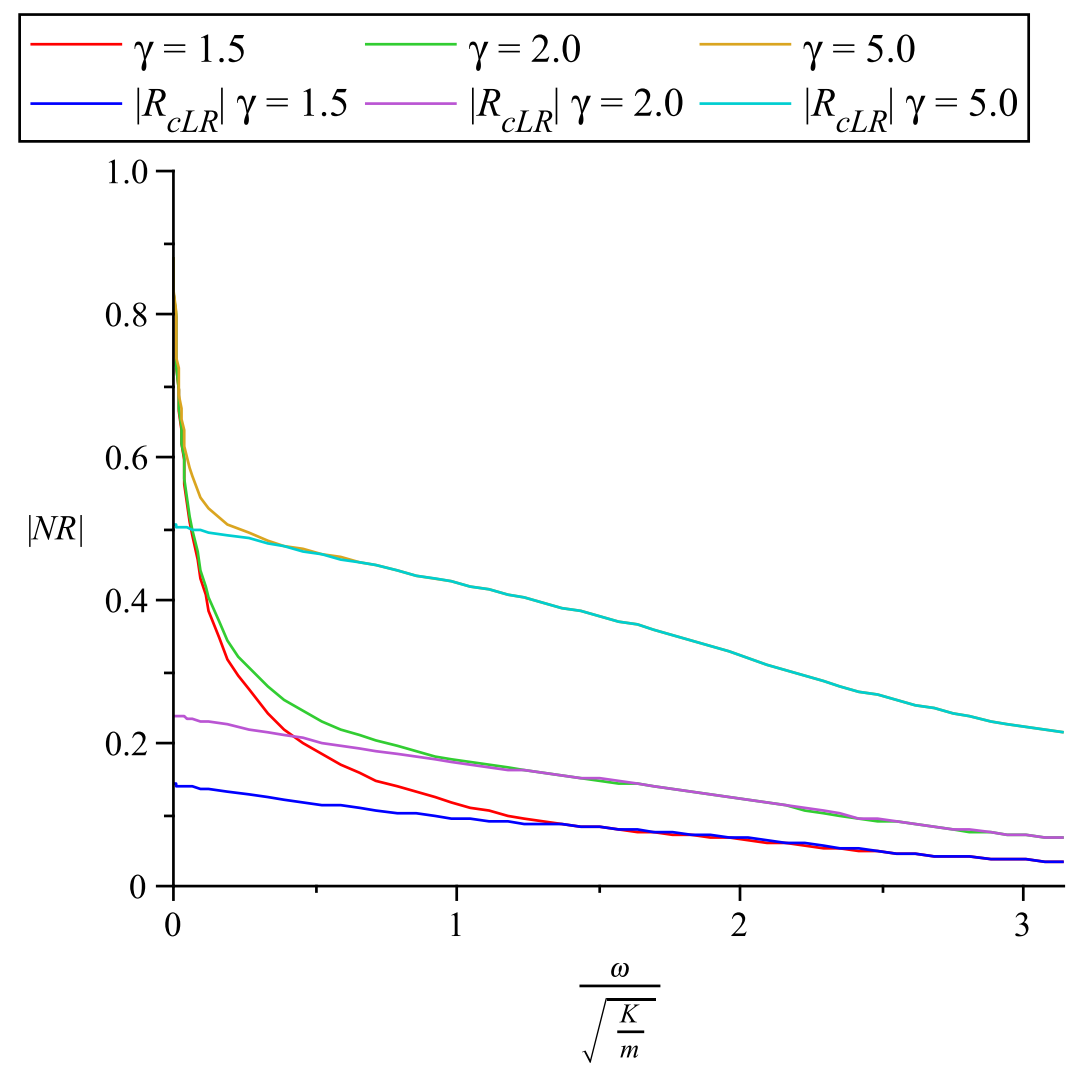

Figure 6.12: This figure shows the net reflectivity $|N R|$ from the damping region as a function of $\omega$ for $\gamma=1$ in the MD region (region $L$ ) and a range of values of $\gamma$ in the damping region (region $R$ ). The corresponding reflectivity from the interface between the MD region and the damping region $\left|R_{c L R}\right|$ is also shown. In this figure $\sqrt{\frac{K}{m}}=1, D=2$, and $a=1$ in both regions.

Figure 6.12 shows that for small values of $\omega$ the net reflectivity back into the MD region is quite high. For all the $\gamma$ values used in this Figure, $|N R|$ trends down to a minimum value of $\left|R_{c L R}\right|$ (the reflectivity from the interface of the MD region and the damping region) as $\omega$ increases. This downward trend can be attributed to the fact that as $\omega$ increases, the imaginary part of the wave number $k_{\text {Rim }}$, and hence the damping, increases.

For each value of $\gamma$ in Figure 6.12, for a sufficiently large value of $\omega,|N R|$ reduces to the level of $\left|R_{c L R}\right|$. Thus from that value of $\omega$ onwards, the portion 
of the wave that is transmitted into the damping region is being damped below 0.01 . The only reflection remaining is from the interface between the MD region and the damping region. In these circumstances, wave reflections from the rigid interface between the damping region and the KMC region have effectively been replaced by reflections from the MD damping region interface.

Note that without a damping effect in the damping region, we would have $|N R|=1$ for all $\omega$.

The level of $\gamma$ considered here, on the order of 2 , is sufficient to extinguish waves when $\omega>1.5$, using a modest damping region of two rows of atoms ( $D=$ 2). In this situation $\gamma=2$ corresponds to a damping number of 4 .

Given the effective damping at this level of $\gamma$, the strategy to mimimse reflections back into the MD region is to utilise the smallest effective $\gamma$, thus exposing the largest portion of incident waves to damping (by maximising transmission into the damping region). This is seen in Figure 6.12, where the case of $\gamma=1.5$ gives the lowest value of $|N R|$ throughout the range of $\omega$.

The next case to consider is when the damping number in the MD region is significantly smaller than 1 . 


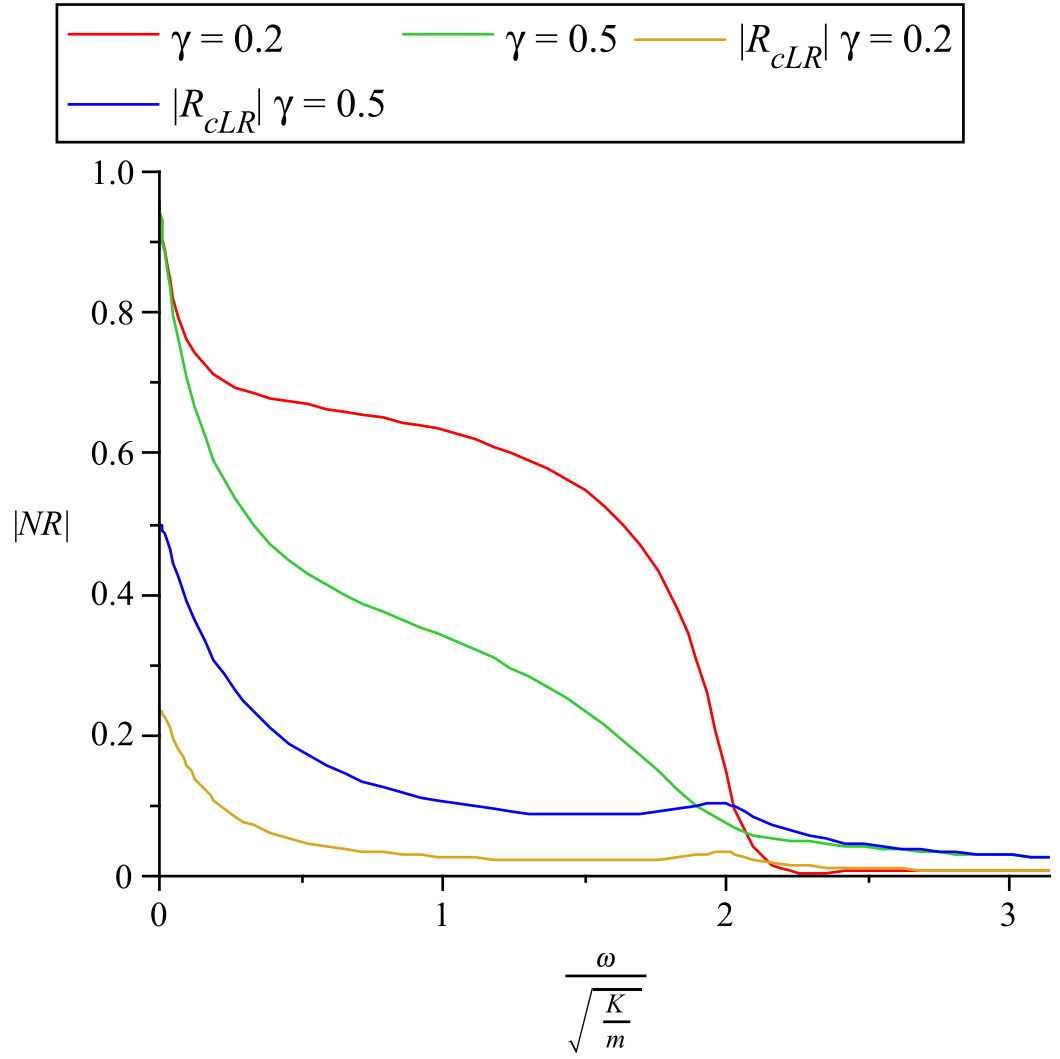

Figure 6.13: This figure shows the net reflectivity $|N R|$ from the damping region as a function of $\omega$, for $\gamma=0.1$ in the MD region (region $L$ ) and a range of values of $\gamma$ in the damping region (region $R$ ). The corresponding reflectivity from the interface between the MD region and the damping region $\left|R_{c L R}\right|$ is also shown. In this figure $\sqrt{\frac{K}{m}}=1, D=2$, and $a=1$ in both regions.

Figure 6.13 shows the net reflectivity $|N R|$ when $\gamma=0.1$ in the MD region. In this case the damping number in the MD region is 0.01 .

In Figure 6.13 small $\gamma$ values of 0.2 and 0.5 are used in the damping region, to get significant transmission into the damping region. However, these small values of $\gamma$ result in weak damping when $\omega$ is less than about 2 . In this case the smallest $\gamma$ value of 0.2 is not the best choice, unlike the situation in Figure 6.12. Setting $\gamma=0.5$ (green curve) in the damping region gives significantly smaller net reflectivity for $\omega \leq 2$ than $\gamma=0.2$ (red curve). For $\omega>2$ the choice of 
$\gamma=0.2$ (red curve) gives slightly less net reflectivity than $\gamma=0.5$ (green curve). The use of the larger $\gamma=0.5$ reduces the transmission into the damping region but this is compensated by greater damping and reduced $|N R|$.

Of course the net reflectivity could be reduced in all cases by lengthening the damping region, i.e. increasing $D$.

\subsection{Hybrid Model with a Damping Region}

In this section we apply the previously developed damping region formula using the parameter values of the hybrid MD KMC model.

For the Lennard-Jones potential used in the hybrid model there is an effective spring constant of $K_{L J}=57.15$, at an interatom distance of $2^{\frac{1}{6}}$ (in reduced units). The Langevin damping parameter $\gamma$ is set to 1 in the MD region. The atom mass is 1 .

Note that with these values $\gamma=1$ corresponds to a very small damping number of 0.017 . Also, the maxmium $\omega$ value for an undamped case is 15.11 . 


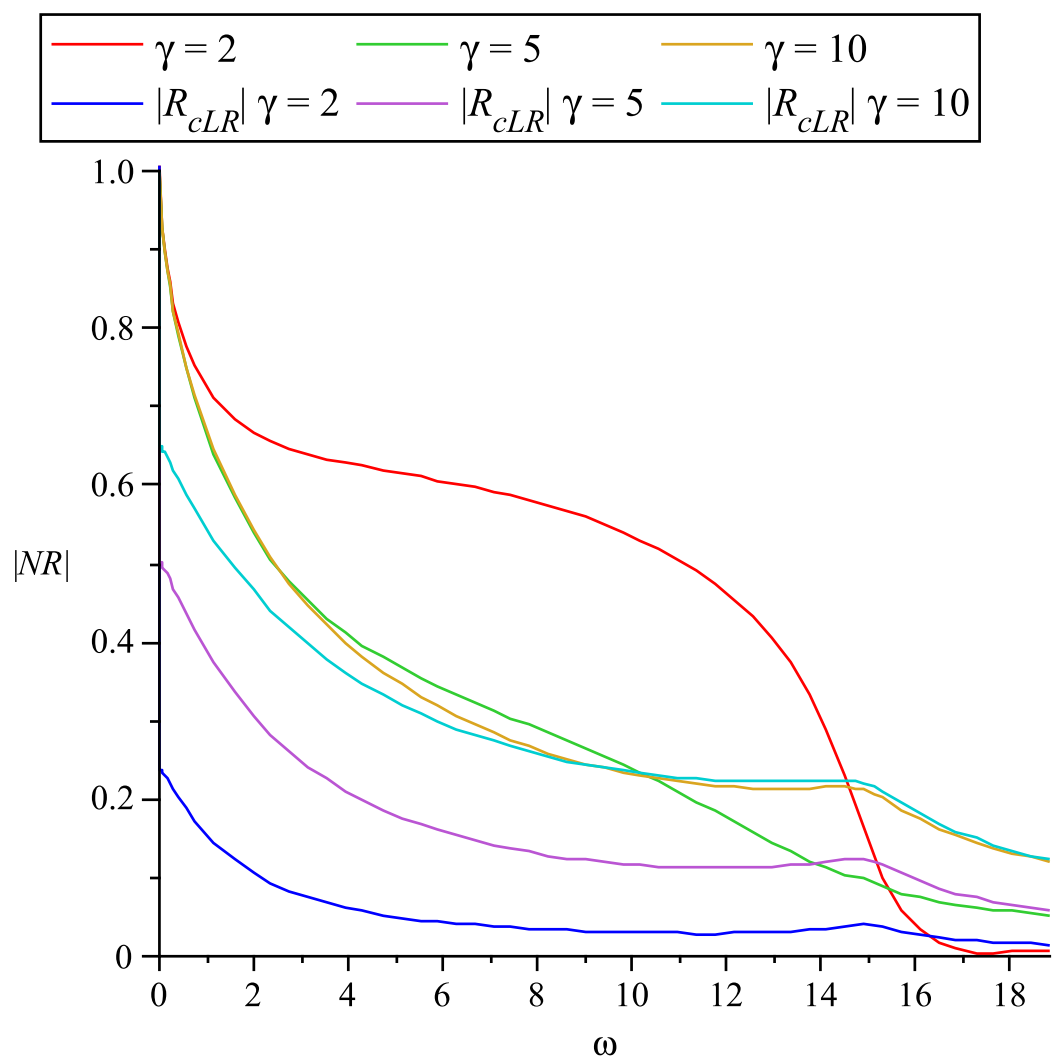

Figure 6.14: This figure shows the net reflectivity $|N R|$ from the damping region as a function of $\omega$ using Lennard-Jones parameter values. $\gamma=1.0$ in the MD region (region $L$ ) and there are a range of values of $\gamma$ in the damping region (region $R$ ). The corresponding reflectivity from the interface between the MD region and the damping region $\left|R_{c L R}\right|$ is also shown. In this figure $K_{L}=57.15$, $K_{R}=57.15, m_{L}=1, m_{R}=1, D=2 \times 2^{\frac{1}{6}}$, and $a=2^{\frac{1}{6}}$ in both regions.

In Figure 6.14 we see the net reflectivity when Lennard-Jones parameter values are used. The choice of a small value of $\gamma=2.0$ in the damping region results in a relatively large value of $|N R|$. When larger $\gamma$ values i.e. 5 and 10 are used there is a significant reduction in $|N R|$ for values of $\omega$ up to about 15 . These larger $\gamma$ values only allow a smaller portion of each wave into the damping region but then damp this portion relatively heavily.

Overall, $\gamma=5.0$ appears the best choice of the $\gamma$ values considered here, as it 
produces a relatively low value of $|N R|$ over a wide range of $\omega$.

However, even with this best choice the overall level of $|N R|$ of approximately 0.30 could still be considered high. We show the effect of doubling the length of the damping region in Figure 6.15.

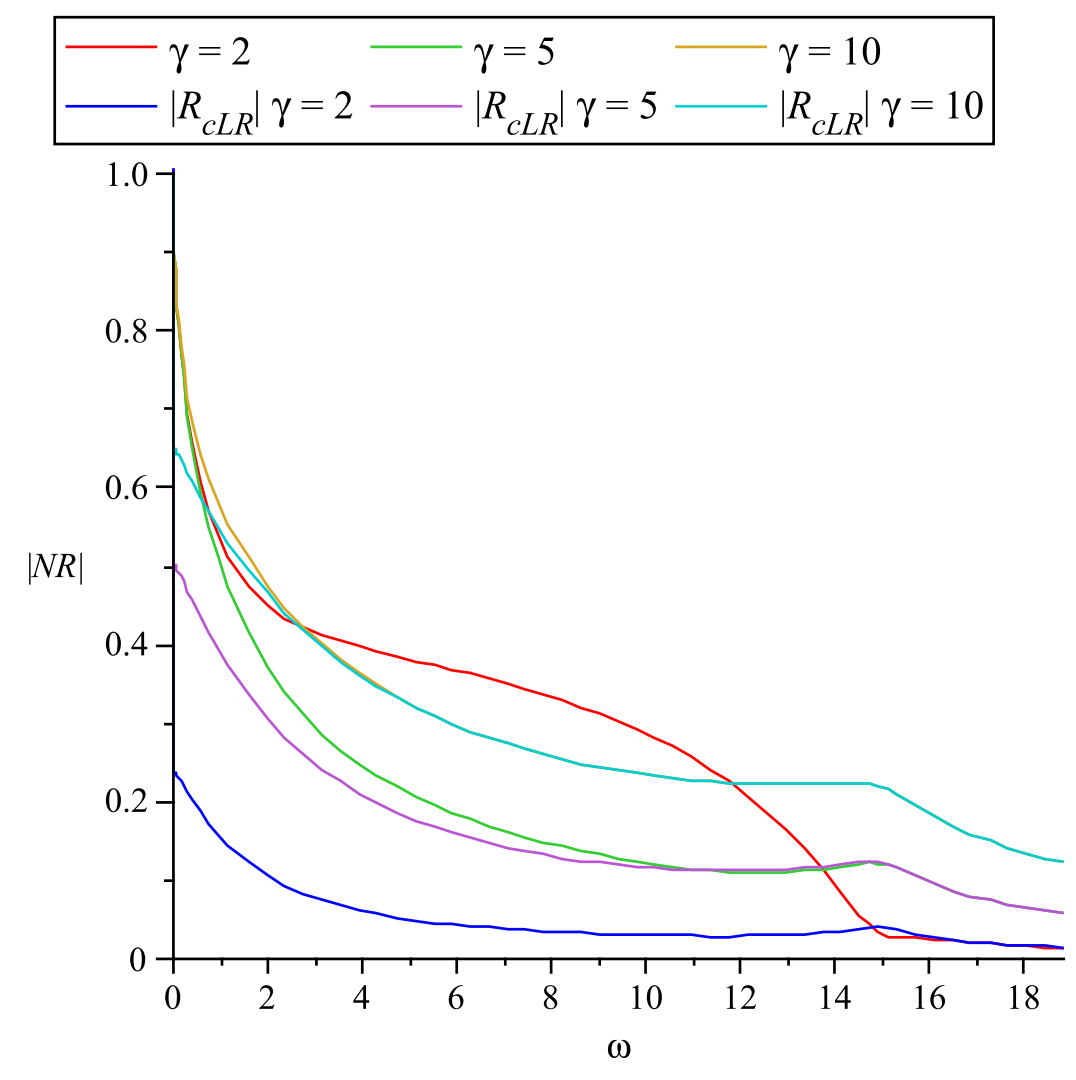

Figure 6.15: This figure shows the net reflectivity $|N R|$ from the damping region as a function of $\omega$ using Lennard-Jones parameter values. $\gamma=1.0$ in the MD region (region $L$ ) and there are a range of values of $\gamma$ in the damping region (region $R$ ). The corresponding reflectivity from the interface between the MD region and the damping region $\left|R_{c L R}\right|$ is also shown. In this figure $K_{L}=57.15$, $K_{R}=57.15, m_{L}=1, m_{R}=1, D=4 \times 2^{\frac{1}{6}}$, and $a=2^{\frac{1}{6}}$ in both regions.

The broad pattern of net reflectivity in Figure 6.14 is repeated in Figure 6.15, but the magnitude of $|N R|$ has been reduced as a result of extending the damping region. In this case also, the choice of $\gamma=5.0$ appears the best of those shown 
giving a relatively low value of $|N R|$ over a wide range of $\omega$. The broad level of $|N R|$ is now approximately 0.20 .

\subsubsection{Selection of $\gamma$ in the Damping Region}

The value of $\gamma$ in the damping region is chosen to minimise the summed net reflectivity $|N R|$, over the range of frequencies that are expected to give rise to wave reflections.

Waves with wavelength less than $30 \sigma$ may be reflected within an MD domain, as the minimum MD domain length is $30 \sigma$. The corresponding wave number is $\frac{2 \pi}{30}=0.209$, and therefore from Eq. (6.2) we determine the minimum frequency of interest as $\omega_{\text {min }}=2 \sqrt{\frac{57.15}{1}} \sin \left(\frac{0.209 \times 2 \frac{1}{6}}{2}\right)=1.77$, using the equilibrium spacing Lennard-Jones effective spring constant of 57.15, and mass of 1 .

A maximum frequency can be determined by considering the maximum frequency for a harmonic oscillator, as previously discussed in Chapter 3. For a harmonic oscillator at the usual simulation temperature of $T=0.35$, the kinetic energy is zero and the potential energy has risen by $3 / 2 \times 0.35=0.525$ at the maximum displacement from the equilibrium position. The Lennard-Jones potential energy rises to $-1+0.525=-0.475$ when the interatom distance has reduced from the equilibrium spacing of $2^{\frac{1}{6}}$ to 1.025 . At this interatom spacing of 1.025, the Lennard-Jones effective spring constant is 303.74, and the corresponding maximum frequency is $\omega_{\max }=\sqrt{\frac{303.74}{1}}=17.43$ for the harmonic oscillator. This value of $\omega_{\text {max }}$ is based on the average kinetic energy at temperature $T=0.35$. However, because the kinetic energy fluctuates above the average value, a larger value of $\omega_{\max }$ is used in this work. We note from Figure 6.14 and Figure 6.15 that as $\omega$ increases beyond $2.0 \sqrt{\frac{K}{m}}=2.0 \sqrt{\frac{57.15}{1}}=15.12$ the reflectivity declines rapidly, as the higher frequencies are heavily damped. Consequently, we set $\omega_{\text {max }}=4.0 \sqrt{\frac{57.15}{1}}=30.24$, as cutting off higher frequencies is not expected to cause any significant error.

Comparisons of the summed $|N R|$ over the frequency range 1.77 to 30.24 for a range of $\gamma$ values in the damping region is shown in Figure 6.16. 


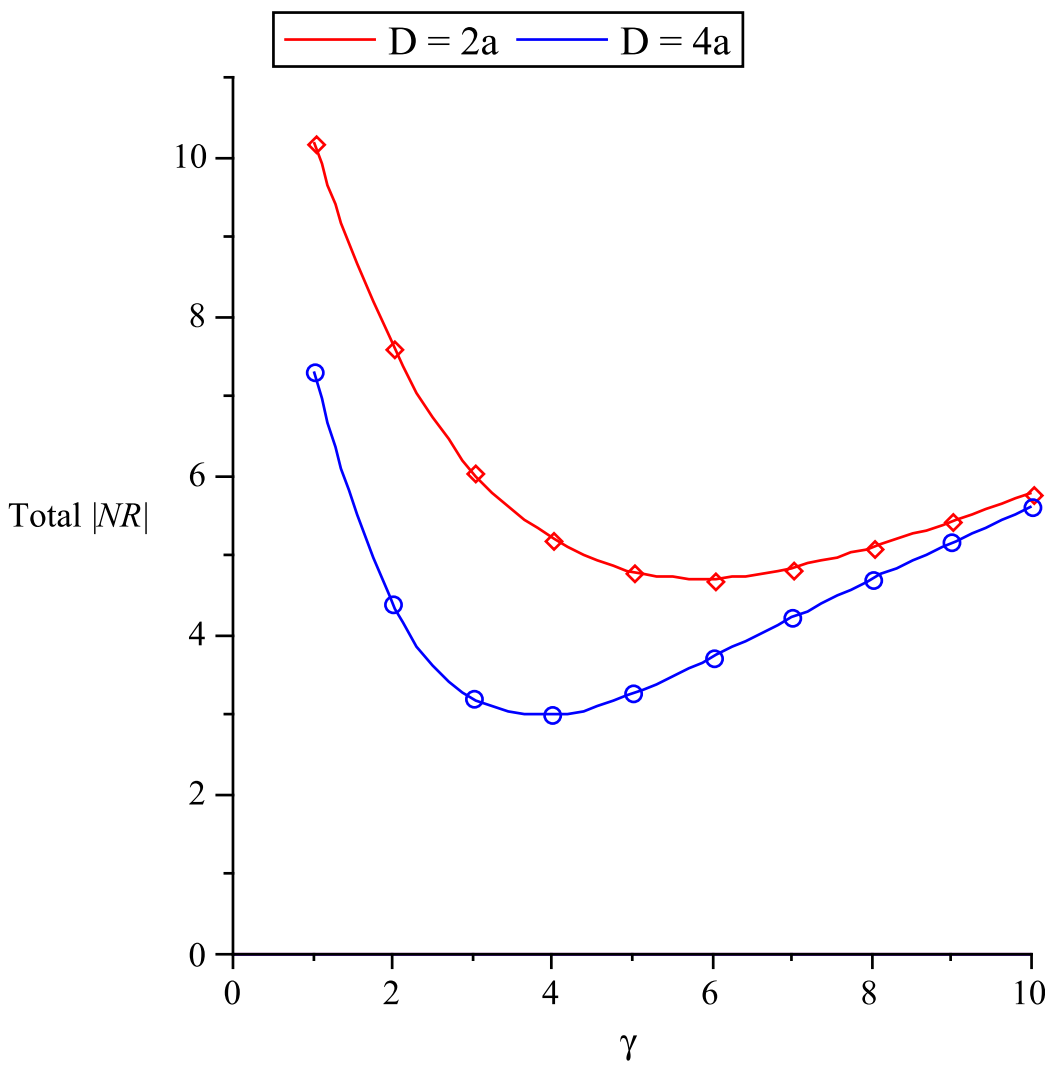

Figure 6.16: This figure shows the net reflectivity $|N R|$, numerically integrated over the frequency range 1.77 to 30.24 for values of $\gamma$ in the damping region (region $R$ ) from 1 to 10 , and two values of $D$. In the MD region (region $L$ ), $\gamma=1$, and in both regions $\sqrt{\frac{K}{m}}=\sqrt{\frac{57.15}{1}}=7.56$ and $a=2^{\frac{1}{6}}$.

When the damping region extends for a distance $2 a$ from the wall of fixed KMC atoms (red curve in Figure 6.16) the greateast damping occurs for $\gamma \approx 6$. For the larger damping region with extent $4 a$ the greatest damping is found when $\gamma \approx 4$. Doubling the extent of the damping region provides distinctly greater damping for $\gamma$ near 4. As $\gamma$ increases the two curves in Figure 6.16 converge, because waves are increasingly being reflected from the interface between the MD region and the damping region, rather than entering the damping region and being attenuated.

On this measure of summed net reflectivity, the use of the larger damping 
region with $\gamma=4$ provides a significant level of damping from a damping region that is modest compared to the size of the MD domain.

\subsection{Summary}

The dispersion relation for a discrete 1-D chain of atoms in the presence of a frictional damping force was derived. This disperion relation is shown in Figure 6.4. To the best of our knowlege this is a new result.

The reflection coefficient formula for wave reflections from an interface between discrete 1-D chains with different material parameters and frictional damping forces was developed. This reflection coefficient formula depends on the dispersion relations in the media on either side of the interface. Therefore the reflection coefficient can be determined for a wave of any frequency $\omega$ incident on the interface.

The formula for the net reflectivity, Eq. (6.9), from a damping region separating an MD region and a KMC region was developed. This is also believed to be a new result.

The effect of the damping region on the magnitude of reflected waves was explored in a number of scenarios. It was shown that for Lennard-Jones parameter values a modest sized damping region can significantly reduce the amplitude of waves reflected back into the MD region by using a damping parameter of $\gamma=$ 4.0. The effectiveness of this damping has not been tested in simulation. 


\section{Chapter 7}

\section{Discussion and Conclusion}




\subsection{Discussion}

The key result of the research described in this thesis is the development and implementation of a hybrid atomistic methodology which dynamically combines both Molecular Dynamics (MD) and Kinetic Monte Carlo (KMC) methodologies within a single simulation. The hybrid method switches the simulation methodology applied to different regions as the simulation progresses, so that MD is only applied to regions where fully detailed atomic trajectories are required while KMC is applied to the other regions. By dynamically partitioning the simulation in this way, the hybrid method provides the same level of detailed results as a corresponding total MD simulation, but at less computational cost. The computational cost saving arises because the more expensive MD is only applied to regions of complex atomic interactions, while less complex regions are adequately treated by much faster KMC. As a result of the reduced computational cost the hybrid method enables simulations with more atoms or for longer periods of simulated time or some combination of both, compared to the MD method.

The detailed feature of this work is the simulation studies of aspects of epitaxial growth. In particular, the behaviour of surface grain boundaries has been studied by separately using MD alone and the hybrid method. This provided a test of the consistency of the hybrid method with comparable MD simulations. It has been demonstrated that when each MD domain in the hybrid model is of length $30 \sigma$ (or more), the dynamics of the grain boundary contained within the MD domain is unaffected by the presence of the adjacent blocks of immobile KMC domain atoms. That is, sufficiently large dynamically reallocated MD domains within the hybrid method reproduce the results of equivalent full MD simulations. The hybrid method also applies a Langevin thermostat to the atoms in each MD domain, to maintain the MD atoms in thermal equilibrium, and to damp the reflection of waves (lattice vibrations) from the interface between each MD domain and adjacent KMC domains. An advantage of the hybrid method is that the MD domains enable observation of effects such as pinning of grain boundaries by atoms in off-lattice sites, which will not be captured by the lattice based KMC. 


\subsubsection{Chapter Summaries}

Chapter 1 introduces epitaxial growth and the importance of epitaxial structures within semiconductor devices, including light emitting diodes and lasers. The investigation of epitaxial growth by simulation is a cost effective way to determine the sensitivity of the growth process to the relevant parameters, and to study growth regimes that are not readily reached by experiments. Modelling of epitaxial growth has a number of difficulties especially the long time scale involved, and a number of hybrid methods have been developed to try and deal with these difficulties.

In Chapter 2 we discuss details of epitaxial growth including applications, homo-and hetero-epitaxy, deposition methods, growth modes and modelling methodologies. A major issue in modelling epitaxial growth is the wide range of length and time scales that play a part in the phenomena. For modelling all aspects of epitaxial growth, neither of the atomistic methods, Molecular Dynamics or Kinetic Monte Carlo, is sufficient. MD cannot address the long time scales involved. KMC may not capture all the important atomic configurational changes. Continuum methods, such as Burton Caberera Frank cover the time scale but ignore important atomistic details. As a result hybrid models such as the model described in this thesis have been developed to address these issues.

Chapter 3 describes the modelling methodologies used in the work. The Molecular Dynamics and Kinetic Monte Carlo simulation methods are discussed. Additional theory used in applying KMC to epitaxial growth simulations, i.e. Transition State Theory and the Nudged Elastic Band method, are also explained.

In Chapter 4 we carry out Molecular Dynamics modelling to set a reference frame for our hybrid model. We show that Molecular Dynamics simulations using the Lennard Jones potential can model dynamic stacking fault grain boundaries in an epitaxial layer on a (111) surface. These simulations reproduce experimentally observed features, e.g. a tendency to form triangular shaped islands. The grain boundaries continually alter their shape and position. The grain boundaries migrate over the surface following a temperature dependent random walk. Over long time periods a small drift in grain boundary position is observed, in addition 
to the random diffusion. A pair of grain boundaries can meet and annihilate each other - eliminating the stacking fault. The key atomic process for grain boundary migration is shown to be the "kink flip" move.

Chapter 5 develops and applies the hybrid MD KMC modelling methodology to the same problem studied with MD in Chapter 4. The hybrid methodology was used to follow the propagation of a stacking fault grain boundary between Face Centered Cubic (FCC) and Hexagonal Close Packing (HCP) islands nucleating on a FCC (111) surface. This hybrid methodology combines Molecular Dynamics and Kinetic Monte Carlo methodologies within a single concurrent atomistic simulation. Different domains within this hybrid simulation are modelled with different methodologies (MD or KMC). And as the simulation progresses the model applied to a given domain is changed (perhaps several times) as necessary, so that the stacking fault grain boundary is always modelled with MD, as it moves about the surface. This hybrid method leads to a speed up over conventional MD, reducing the execution time by a factor approximately equal to the relative fraction of remaining MD atoms. We show that it is possible to reproduce grain boundary mobilities from full MD simulations with the domain decomposition method in the absence of KMC events - when the MD regions are sufficiently large with length $30 \sigma$. We have also used the method to study the effect of over layer adatoms on the mobility of the boundaries, demonstrating that boundaries can become trapped by adatom islands. These effects would have been difficult to capture in a conventional KMC simulation.

In Chapter 6 a way to enhance the hybrid model by reducing wave (lattice vibration) reflections from the MD KMC interface is investigated. It is shown that the reflection coefficient is a function of the Langevin damping parameter $\gamma$. It is proposed that by suitable tuning of $\gamma$ in the damping region adjacent to the rigid KMC atoms, the reflections of waves back into the MD domain can be minimised. Such a reduction in wave reflections implies that a smaller MD domain will be sufficient, thus further speeding up the hybrid model. This is not investigated in detail but left for future development. 


\subsection{Conclusion}

This thesis has developed a hybrid atomistic simulation method which dynamically combines the Molecular Dynamics and Kinetic Monte Carlo methodologies. With suitably selected MD domains this hybrid method can reproduce the results of a larger (more atoms) all MD simulation at a significant reduction in computational cost (run time). This reduction is related to the proportion of all MD simulation atoms which have been replaced by KMC atoms in the hybrid model.

This hybrid method offers another approach to computer simulation of phenomena such as epitaxial growth, which are difficult to treat because of the wide range of length and time scales involved.

The hybrid method was successfully used to study the dynamics of stacking fault grain boundaries in epitaxial surface layers. The hindering of grain boundary motion due to pinning by deposited adatoms and also by the formation of overlayer islands was identified using the hybrid model. These results could not be captured by KMC alone as pinning adatoms were located in an off-lattice sites. While MD alone could not have simulated the nucleation and growth of overlayer islands within a feasible runtime.

In the process of developing the hybrid model we have verified that the 'kink flip" move is a key element in the motion of grain boundaries.

In the final part of this thesis we have investigated an approach to further speed up the hybrid model. We have developed a mathematical model of the effect of the damping parameter $\gamma$ on the reflection of lattice vibration waves from the interface between MD domains and KMC domains.

This mathematical modelling included the derivation of the dispersion relation for the discrete case with damping and net reflectivity formulas. These are believed to be new results.

It was shown that by an optimal choice of $\gamma$ wave reflections can be minimised. Reduced wave reflections back into the MD domain imply that a smaller MD domain would suffice in the hybrid model. A smaller MD domain makes larger and/or longer hybrid model simulation runs feasible. This improvement to the 
hybrid model has not been implemented, but left for future development.

Another possible future development of this approach to damping wave reflections is to use either a stepped series of damping regions or a gradient in the damping parameter $\gamma$. Such an arrangement may increase the amount of damping that can be obtained.

The hybrid model has been developed and tested on a long narrow slab geometry. The next logical future extension is to extend the hybrid model to a full two dimensional surface. In principal the hybrid model could be further extended to a three dimensional volume. Each such extension would be a significant research project in its own right. A key aspect for any such further extension is expected to the development of methods to efficiently identify changes in grain boundary shape and to track changes grain boundary position, in two and three dimensions.

This hybrid model is another tool in the collection of multiscale methods. It has been viewed as a method to speed up MD but it can also be considered as a method to enable KMC to handle more complexity.

The hybrid model can also be applied to a wide variety of MD and KMC methods. For example other MD potentials such as Embedded Atom or Modified Embedded Atom could be employed. It may be feasible to incorporate Ab-initio MD methods. The KMC component can be developed to use a more refined lattice enabling more complicated configuration changes in the KMC domain. An "on the fly" KMC method could be employed, where hop rates for configuration changes are developed as the run proceeds. This eliminates the need for a predetermined table of hop rates but increases the computational cost of KMC. Both the MD and KMC components can be extended to handle more than one species of atom. A further enhancement is to develop parallelised versions of the MD and KMC components, so that computer clusters or multicore computers can be used to speed up the run time or enable larger simulations. 


\section{Bibliography}

[1] L Royer. Bull. Soc. Fr. Miner. Crystallog., 51:7, 1928.

[2] I Markov, E Stoycheva, and D Dobrev. Commun. Dept. Chem. Bulg. Acad. Sci., 3:377, 1978.

[3] P M J Maree, J C Barbour, J F van der Veen, and K L Kavanagh. Surf. Sci, 191:305, 1987.

[4] A Usui and H Watanabe. Ann. Rev. Mater. Sci., 21:185, 1991.

[5] D W Pashley. Adv, Phys., 5:173, 1956.

[6] H C Theurer. J. Electrochem. soc, 108:649, 1961.

[7] L Esaki and R Tsu. IBM J. Res. Develop., 14:61, 1970.

[8] W Dingle, W Wiegmann, and C H Henry. Phys. Rev. Lett, 38:827, 1974.

[9] B Johnstone. Compound Semiconductor, 1:36, 1995.

[10] S Nakamura and G Fasol. The Blue Laser Diode. Springer Verlag, Berlin, 1997.

[11] S Nakamura. Ann. Rev. Mater. Sci., 28:125, 1998.

[12] Y Arakawa and A Yariv. IEEE J Quantum Electron., QE 22:1887, 1986.

[13] H Sakaki. Jpn. J. Appl. Phys., 19:L735, 1987. 
[14] B Binasch, P Grunberg, F Sauerenbach, and W Zinn. Phys. Rev. B, 39:4828, 1989.

[15] M N Baibich, J M Broto, A Fert, F Nguyen Van Dau, and F Petroff et al. Phys. Rev. Lett., 61:2472, 1988.

[16] E Wigner. Phys. Rev., 46:1004, 1934.

[17] C Mellor. New Scientist, 135:1833, 1992.

[18] K von Klitzing, G Dorda, and M Pepper. Phys. Rev. Lett., 45:494, 1980.

[19] D C Tsui, H L Störmer, and A C Gossard. Phys. Rev. Lett., 48:1559, 1982.

[20] J M Pomeroy, J Jacobsen, C C Hill, B H Cooper, and J P Sethnal. Phys. Rev. B, 66:235412, 2002.

[21] T P Schulze, P Smereka, and W E. J. Comp. Phys., 189:197-211, 2003.

[22] T P Schulze. J. Cryst. Growth, 263:605-615, 2004.

[23] I. V. Markov. World Scientific, New Jersey, 2004.

[24] M. Ohring. Academic Press, San Diego, 2002.

[25] J M Hartmann, Y Bogumilowicz, F Andrieu, P Hollinger, G Rolland, and T Billon. J. Cryst. Growth, 277:114, 2005.

[26] H Amano, N Sawaki, I Akasaki, and Y Toyoda. Appl. Phys. Lett., 48:353, 1986.

[27] A G Baca and C I H Ashby. Fabrication of GaAs Devices. The Institution of Electrical Engineers, London, 2005.

[28] S A Campbell. The Science and Engineering of Microelectronic Fabrication. Oxford University Press, 2001.

[29] B Voigtländer. Surf. Sci. Rep., 43:127, 2001. 
[30] W I Wang. Appl. Phys. Lett., 44:1149, 1984.

[31] I B Bhat, N R Tasker, and S K Ghandhi. J. Vac. Sci. Technol. A, 4:2230, 1986.

[32] C P Kuo, J S Yuan, R M Cohen, J Dunn, and G B Stringfellow. Appl. Phys. Lett., 44:550, 1984.

[33] S Nakamura. J. Mater. Res., 14:2716, 1999.

[34] D J Paul. Adv. Mater., 11(3):191, 1999.

[35] S M Sze, editor. Modern Semiconductor Device Physics. J. Wiley \& Sons, New York, 1998.

[36] Special-issue. Quantum devices and applications. Proc. IEEE, 87(4):535, 1999.

[37] F Capasso and G Margaritondo, editors. Band Discontinuities: Physics and Device Applications. Elsevier, Amsterdam, 1990.

[38] M Razeghi. Technology of Quantum Devices. Springer, New York, 2010.

[39] O Auciello, C M Foster, and R Ramesh. Ann. Rev. Mater. Sci., 28:501, 1998.

[40] R P Ruth, J C Marinace, and W C Dunlap. J. Appl. Phys., 31:995, 1960.

[41] A L Robinson. Science, 188:720, 1975.

[42] T V Barker. J. Chem. Soc. Tran., 89:1120, 1906.

[43] J Narayan. Metallurgical and Materials Transactions B, 36B:5, 2005.

[44] C F Powell and J H Oxley. Vapor Deposition. The Electrochemical Society series New York Wiley, 1966.

[45] R Behrisch. Sputtering by Particle bombardment. Springer Berlin, 1981. 
[46] M B Panish and H Temkin. Ann. Rev. Mater. Sci., 19:209, 1989.

[47] G B Stringfellow. Organometallic Vapor-Phase Epitaxy. Academic, Boston, 1989.

[48] B A Joyce and R R Bradley. Phil. Mag., 14:289, 1966.

[49] A Y Cho and J R Arthur. Molecular beam epitaxy. Prog. Solid State Chem., 10:157-192, 1975.

[50] A Ichimiya and P I Cohen. Reflection High Energy Electron Diffraction. Cambridge University Press, Cambridge, 2004.

[51] G Binnig and $\mathrm{H}$ Rohrer. Scanning tunneling microscopy. IBM J. Res. Develop., 30:4, 1986.

[52] H A Atwater, C C Ahn, S S Wong, G He, H Yoshino, and S Nikzad. Energyfiltered rheed and reels for in situ real time analysis during film growth. Surf. Rev. and Lett., 4:525, 1997.

[53] B Voigtländer and A Zinner. Appl. Phys. Lett., 63:3055, 1993.

[54] C Pearson, M Kruegur, and E Grantz. Phys. Rev. Lett., 76:2306, 1996.

[55] E Bauer. Z. Kristallographie, 110:372, 1958.

[56] M Volmer and A Weber. Z. Phys. Chem., 119:277, 1926.

[57] F C Frank and J H van der Merwe. Proc. Roy. Soc. London, A198:205, 1949.

[58] I N Stranski and L Krastanov. Stizungsber. Akad. Wissenschaft Wein, 146:797, 1938.

[59] V A Shchukin, N N Ledenstov, and D Bimberg. Epitaxy of Nanostructures. Springer, Berlin, 2004.

[60] I Markov and S Stoyanov. Contemp. Phys., 28:267, 1987. 
[61] R L Schwoebel. J. App. Phys., 37:3682, 1966.

[62] J Villain. J. Phys. I, 1:19, 1991.

[63] D D Vvedensky. Epitaxial Nanostructures across Length and Time Scales. The Blackett Laboratory Imperial College London, 2002.

[64] M. P. Allen and D. J. Tildesley. Oxford Science Publications, Oxford, 1987.

[65] A F Voter. Radiation Effects in Solids, chapter Introduction to the Kinetic Monte Carlo Method. Springer, 2005.

[66] W. K. Burton, N. Cabrera, and F. C. Frank. The growth of crystals and the equilibrium structure of their surfaces. Phil. Trans. Roy. Soc. London Ser. A, 243(1951):299, 1951.

[67] A Pimpinelli and J Villain. Physics of Crystal Growth. Cambridge University Press, Cambridge, 1998.

[68] R Ghez and S S Iyer. IBM J. Res. Develop., 32:804, 1988.

[69] G Mandel. J.Chem. Phys., 40:683, 1964.

[70] R E Caflisch, W E, M F Gyure, B Merriman, and C Ratsch. Phys. Rev. E, 59:6879, 1999.

[71] R E Caflisch, M F Gyure, B Merriman, S Osher, C Ratsch, D D Vvedensky, and J J Zinck. Appl. Math. Lett., 12:13-22, 1999.

[72] R E Caflisch and Li B. Multiscale Model. Sim., 1:150-171, 2002.

[73] S Chen, B Merriman, S Osher, and P Smereka. J. Comp. Phys., 135:8, 1997.

[74] S Osher and J A Sethian. J. Comp. Phys, 79:12, 1988.

[75] Y M Yu and B G Liu. Phys. Rev. E, 69:21601, 2004. 
[76] T S Lo and R V Kohn. Physica D, 161:237, 2002.

[77] A L Barabasi and H E Stanley. Fractal Concepts in Surface Growth. Cambridge University Press, Cambridge, 1995.

[78] R Lam and D G Vlachos. Phys. Rev. B, 64:35401, 2001.

[79] J Kundin and H Emmerich. Eur. Phys. J. B, 63:25, 2008.

[80] P Zoontjens, T P Schulze, and S C Hendy. Phys. Rev. B, 76:245418, 2007.

[81] H Trebin, R Mikulla, J Stadler, G Schaaf, and P Gumbsch. Comp. Phys. Comm., 121:536-539, 1999.

[82] H Waisman and J Fish. Comp. Meth. Appl. Mech. and Eng., 195:65426559, 2006.

[83] X Yin, A Endou, R M, A Fahmi, I Gunji, R Yamauchi, M Kubo, K Teraishi, and A Miyamoto. Comp. Mat. Sci., 14:114-118, 1999.

[84] Y Gurler and S Ozgen. Mat. Lett., 57:4336-4343, 2003.

[85] C L Brooks. Curr. Op. Struct. Biol., 3:92-98, 1993.

[86] L J Alvarez, J F Sanz, M J Capitan, and J A Odriozola. J. Mol. Struct., 287:161-166, 1993.

[87] M Souaille, H Loirat, D Borgis, and M P Gaigeot. Comp. Phys. Comm., 180:276-301, 2009.

[88] D P Tieleman, W F Drew Bennet, and J MacCallum. Chem. Phy. Lip., 149:S4, 2007.

[89] D L Beveridge and G Ravishanker. Curr. Opin. Struct. Biol., 4:246-255, 1994.

[90] C Amano, H Niina, and Y Mikami. J. Mol. Struct., 904:64-68, 2009. 
[91] K Maekawa and A Itoh. Wear, 188:115-122, 1995.

[92] A Tsukamoto, K Nakagawa, and A Itoh. J. Magnet. Magnet. Mat., 198:710-712, 1999.

[93] M J Buehler. Atomistic Modeling of Materials Failure. Springer, New York, 2008.

[94] T C Germann and K Kadau. Intl. J. Mod. Phys. C, Vol. 19, No. 9:13151319, 2008.

[95] R K Pathria. Statistical Mechanics. Pergamon Press, 1972.

[96] G S Grest and K Kremer. Phys. Rev. A, 33:3628, 1986.

[97] H B Callen and T A Welton. Phys. Rev., 83:34, 1951.

[98] J Weber. Phys. Rev., 101:1620, 1956.

[99] N W Ashcroft and N D Mermin. Solid State Physics. Holt, Rinehart and Winston, New York, 1976.

[100] N Bernades. Phys. Rev., 112:1534, 1958.

[101] A Rahman. Phys. Rev., 136:A 405, 1964.

[102] C Kittel. John Wiley \& Sons, New York, 1971.

[103] P.M. Morse. Diatomic molecules according to the wave mechanics. iivibrational levels. Phys. Rev., 34:57-64, 1929.

[104] R. Komanduri, N. Chandrasekaran, and L.M. Raff. Molecular dynamics (md) simulations of uniaxial tension of some single-crystal cubic metals at nanolevel. Int. J. Mech. Sci., 43:2237-2260, 2001.

[105] M.I. Baskes. Embedded-atom method: Derivation and application to impurities, surfaces and other defects in metals. Phys. Rev. B, 29(12):64436543, 1984. 
[106] M. W. Finnis and J. E. Sinclair. A simple empirical n-body potential for transition metals. Phil. Mag., 50:45-55, 1984.

[107] Y. Mishin, M.J. Mehl, D.A. Papaconstantopoulos, A.F. Voter, and J.D. Kress. Structural stability and lattice defects in copper: Ab-initio, tightbinding and embedded-atom calculations. Phys. Rev. B, 63:224106, 2001.

[108] J.G. Swadener, M.I. Baskes, and M. Nastasi. Molecular dynamics simulation of brittle fracture in silicon. Phys. Rev. Lett., 89(8):085503, 2002.

[109] A K Mazur. J. Comp. Phys., 136:354-365, 1997.

[110] G D Venneri and W G Hoover. J. Comp. Phys., 73:468-475, 1987.

[111] G Zhang and T Schlick. J. Comp. Chem., 14:1212-1233, 1993.

[112] G Zhang and T Schlick. J. Chem. Phys., 101:4995, 1994.

[113] D W Heermann. Computer Simulation Methods. Springer, 1990.

[114] D Frenkel and B Smit. Understanding Molecular Simulation. Academic Press, 2002.

[115] L. Verlet. Computer experiments on classical fluids. i. thermodynamical properties of lennard-jones molecules. Phys. Rev., 159:98, 1967.

[116] gopenmol. www.chem.utah.edu/faculty/anderson/gopen.html.

[117] M J Buehler, H Tang, A C T van Duin, and W A Goddard. Phys. Rev. Lett., 99:165502, 2007.

[118] M J Buehler, A C T van Duin, and W A Goddard. Phys. Rev. Lett., 96:095505, 2006.

[119] H. Eyring. The activated complex in chemical reactions. J. Phys. Chem., 3(107), 1935.

[120] S. Glasstone, K. J. Laidler, and H. Eyring. McGrowHill, New York, 1941. 
[121] C. A. Wert and C. Zener. Interstitial atomic diffusion coefficients. Phys. Rev., 76:1169, 1949.

[122] G. H. Vineyard. Frequency factors and isotope effects in solid state rate processes. J. Phys. Chem. Solids, 3(121), 1957.

[123] Hanggi, Talkner, and Borkovec. Reaction-rate theory: fifty years after kramers. Rev. Mod. Phys., 62(2):251, 1990.

[124] P. Pechukas. Dynamics of molecular collisions, Part B (W. H. Miller ed.). Plenium Press, New York, 1976.

[125] D. G. Truhlar, W. L. Hase, and J. T. Hynes. Current status of transition-state theory. J. Phys. Chem., 87:2664, 1983.

[126] D. G. Truhlar, B. C. Garret, and S. J. Klipenstein. Current status of transition-state theory. J. Phys. Chem., 100:12771, 1996.

[127] S. Clarke and D. D. Vvedensky. Origin of reflection high-energy electrondiffraction intensity oscillations during molecular-beam epitaxy: A computational modeling approach. Phys. Rev. Lett., 2235(58), 1987.

[128] H. Jónsson, G. Millis, and K. W. Jacobsen. World scientific, 1998.

[129] G. Millis and H. Jónsson. Quantum and thermal effects in h2 dissociative adsorption: Evaluation of free energy barriers in multidimensional quantum system. Phys. Rev. Lett., 72(1124), 1994.

[130] G. Millis, H. Jónsson, and G. Schenter. Reversible work transition state theory: Application to dissociative adsorption of hydrogen. Sur. Sci., 324:305, 1995.

[131] G. Henkelman and H. Jónsson. Improved tangent estimate in the nudged elastic band method for finding the minimum energy paths and saddle points. J. Chem. Phys., 9978(113), 2000. 
[132] G. Henkelman, B. P. Uberuaga, and H. Jónsson. A climbing image nudged elastic band method for finding saddle points and minimum energy paths. J. Chem. Phys., 113(9901), 2000.

[133] A F Voter. J. Chem. Phys., 106:4665, 1997.

[134] A F Voter. Phys. Rev. B, 57:13985, 1998.

[135] M R Sorensen and A F Voter. J. Chem. Phys., 112:9599, 2000.

[136] A Kushima, X Lin, J Li, X Qian, J Eapen, J C Mauro, P Diep, and S Yip. J. Chem. Phys., 130:224504, 2009.

[137] T T Lau, A Kushima, and S Yip. IOP Conf. Series: Material Science and Engineering, 3:012002, 2009.

[138] A. B. Bortz, M. H. Kalos, and J. L. Lebowitz. J. Comp. Phys., 17(10), 1975.

[139] H. C. Kang and W. H. Weinberg. J. Chem. Phys., 90(2824), 1989.

[140] M E J Newman and G T Barkema. Monte Carlo Methods in Statistical Physics. Oxford University Press, 1999.

[141] K. Binder. (Springer topics in current physics 7). Springer, Berlin:, 1979.

[142] K. Binder and D. W. Heermann. (series in solid-state science 80). Springer, Berlin:, 1988.

[143] K. A. Fichthorn and W. H. Weinberg. Theoretical fundations of dynamical monte carlo simulations. J. Chem. Phys., 95:1090 - 6, 1991.

[144] C Busse, C Polop, M Müller, K Albe, U Linke, and T Michely. Phys. Rev. Lett., 91:056103, 2003.

[145] W L Ling, N C Bartelt, K F McCarty, and C B Carter. Phys. Rev. Lett., 95:166105, 2005. 
[146] C Busse and T Michely. Surf. Sci., 552:281-293, 2004.

[147] A. C. To and S. Li. Phys. Rev. B, 72:035414, 2005.

[148] W E and Z Huang. Phys. Rev. Lett., 87:135501, 2000.

[149] W Cai, M de Koning, V V Bulatov, and S Yip. Phys. Rev. Lett., 85:3213, 2000. 